\title{
37. UPPER CRETACEOUS TO QUATERNARY SEDIMENTARY PROCESSES IN THE BAY OF BISCAY FROM TEXTURAL, MINERALOGICAL, AND COARSE FRACTION STUDIES ${ }^{1}$
}

\author{
G.A. Auffret and L. Pastouret, Centre Océanologique de Bretagne, B.P. 337, 29273 Brest Cedex, France
}

\begin{abstract}
The evolution of the sedimentary regime that prevailed on the Armorican margin from Late Cretaceous to late Tertiary is herein discussed and interpreted. The sedimentation budget of four components of the sediment: calcium carbonate, quartz, "less than $10 \mu \mathrm{m}$ non carbonate material," and biogenic silica are quantified as is the composition of the coarse fraction and the textural properties of the sediment. Variation of the coarse fraction components is directly related to change in the oceanic environment (terrigenous supplies, productivity, calcium carbonate dissolution). Sediment texture is a function of many factors, comprising lithology, dissolution action, and hydrodynamic control. The medians of the silt fractions from bulk and decalcified sediments are used as indicators of variations of the bottom current intensity averaged over long periods $\left(\mathrm{n} \times 10^{2}\right.$ to $\left.\mathrm{n} \times 10^{3} \mathrm{yr}\right)$ of time. From the study the following aspects of the evolution of the sedimentary regime are characterized:

1) establishment of an active bottom current circulation during the early Eocene, concomitant with a clear thermal stratification of the water masses;

2) development of a highly fluctuating bottom current regime during the Oligocene, spicule-rich "contourites" being implaced during periods of active bottom currents;

3) changes in the sedimentary regime at the early-middle Miocene boundary characterized by decreasing bottom current and increasing sedimentation rates;

4) active bottom current, decreasing dissolution and marked increase of the sedimentation rates in the early Pliocene, that contributed to active building of a sedimentary ridge at the foot of the margin;

5) it appears that the lowermost Upper Cretaceous hiatus and the upper Eocene hiatus are related to changes in the physiographic (and hydrologic ?) environment at an oceanic rather than regional scale.
\end{abstract}

\section{INTRODUCTION}

An attempt is made within this paper to interpret the evolution of the sedimentary regime that prevailed on the Armorican margin (Figure 1) from Late Cretaceous to Quaternary time. For that purpose, new methodologies have been used to quantify the sedimentation rates of four components of the sediment (calcium carbonate, quartz, "less than $10 \mu \mathrm{m}$ non-carbonate material," and biogenic silica), to interpret the nature of sedimentary regime through visual examination and quantitative study of the coarse fraction of the sediment and to infer possible fluctuations of the bottom current regime from textural study of the silt fractions. Analytical procedures are described and the sedimentary sections at Sites 402,401 , and 400 are interpreted and, finally, the sedimentary evolution of the margin is considered.

'Contribution 599 du Département Scientifique, Centre Océanologique de Bretagne.

\section{ANALYTICAL PROCEDURES}

\section{General Procedures}

Samples of 5 to $10 \mathrm{~cm}^{3}$ of sediment were taken from cores on board R/V Glomar Challenger, with particular attention being paid to sedimentary structures that bore evidence of possible current actions. Following visual examination of the sample under the binocular microscope, the samples were split longitudinally into two parts, one being kept as archive. Smear slides and sediment color evaluation were made on the second half, which was then dried at about $60^{\circ} \mathrm{C}$ in an oven. The dried sample was again split into two parts. One was wet-sieved through a $63-\mu \mathrm{m}$ mesh. The coarse fraction was dried, weighed, and sieved (when it exceeded $1 \%$ of the total sample). The suspension containing the finer (less than $63 \mu \mathrm{m}$ ) particles was evaporated and size distribution analysis was made of the residue. The other half was crushed in an agate mortar, and $0.250 \mathrm{~g}$ examined for calcium carbonate content (Bernard calcimeter); $0.200 \mathrm{~g}$ was used for X-ray diffraction analyses with an $\mathrm{NaF}$ internal standard. 


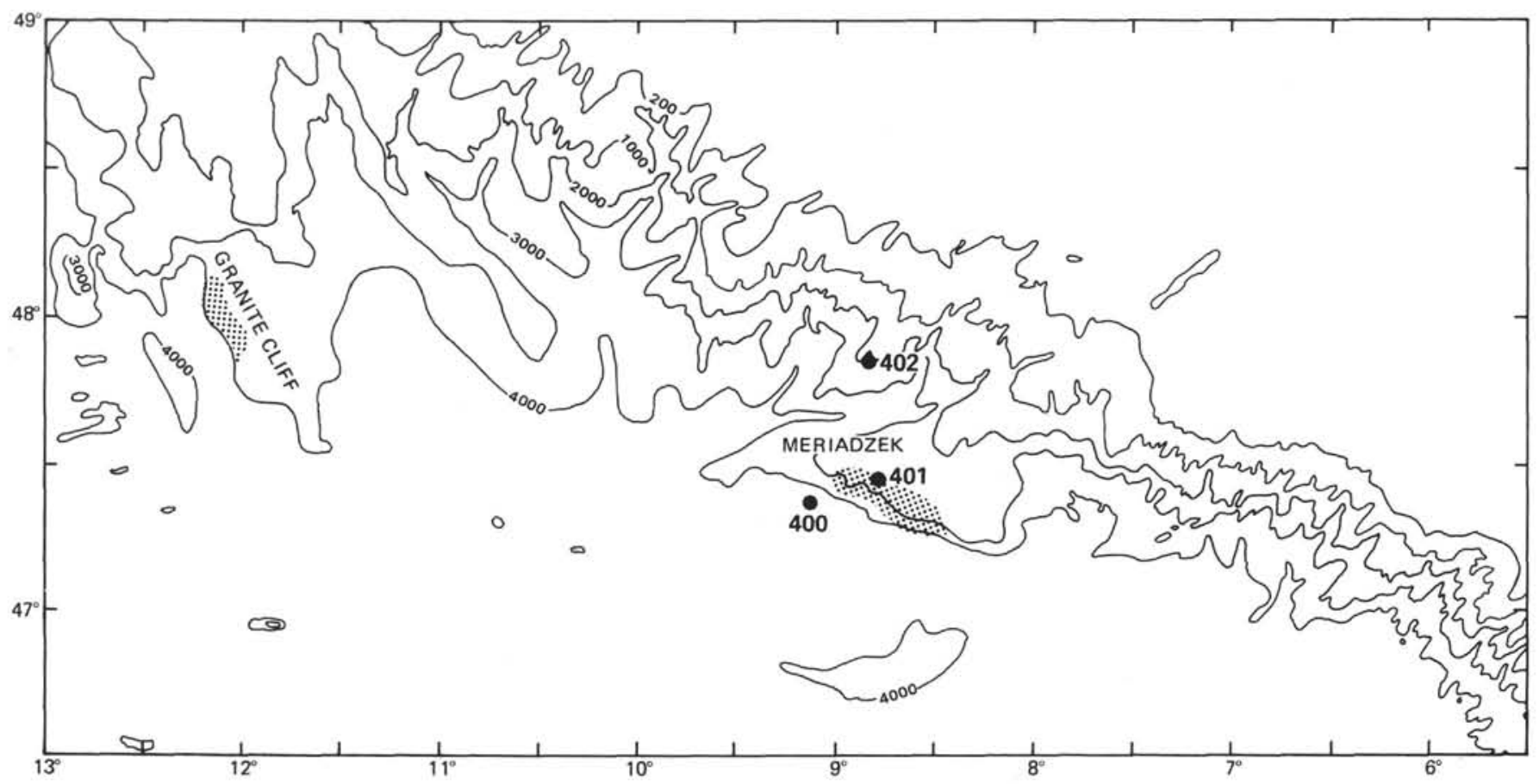

Figure 1. Bathymetric chart of the Armorican and Celtic margins with Upper Cretaceous condensated section.

\section{Coarse Fraction Study}

The sediment fraction coarser than $63 \mu \mathrm{m}$ was separated by dry sieving into five classes; $63-125 \mu \mathrm{m}$, very fine sand; 125-250 $\mu \mathrm{m}$, fine sand; $250-500 \mu \mathrm{m}$, medium sand; 500-1000 $\mu \mathrm{m}$, coarse sand; $>1000 \mu \mathrm{m}$, very coarse sand. Quantitative evaluations of the different components (Table 1) were then made on the reconstituted samples, by counting approximately 500 grains that had been separated with a microsplitter. From these counts the different percentages were computed. The planktonic foraminifer/ benthic foraminifer $(\mathrm{PF} / \mathrm{BF})$ ratio was calculated and the state of preservation of the calcareous biogenic components (principally foraminifer) estimated.

\section{Micrograin Size Distribution Analyses}

Particle grain size distribution analyses were performed with an electronic particle counter (Coulter counter TA type), the measured particle dimension being the volume diameter, that is, the diameter of a sphere of equivalent volume. The range of dimensions lay between 1.7 to 56 $\mu \mathrm{m}$, but the texture of the silt fraction $(8-56 \mu \mathrm{m})$ in particular was emphasized.

For study of the $<63 \mu \mathrm{m}$ fraction, $9 \mathrm{~cm}^{3}$ were sampled from the suspension while it was submitted to mechanical agitation; dilution in about $250 \mathrm{~cm}^{3}$ of water followed. Fifty $\mathrm{cm}^{3}$ of the diluted suspension was then mixed with $150 \mathrm{~cm}^{3}$ of filtered sea water, the entire procedure being repeated three times. The same procedure was done on the decalcified sediments. Nine $\mathrm{cm}^{3}$ of the suspension were treated with $\mathrm{HCl}(30 \%)$. After reaction, the sample was washed and centrifuged three times with distillated water. At the end of the washing, $p \mathrm{H}$ values were about 7 to 8 .

Sediment texture is a function of many factors, including lithological composition (Lisitzin, 1972); syn- and postdepositional transformations (Berger, 1974; Calvert, 1974;
Kagami, this volume); and hydrodynamic control (Hollister and Heezen, 1972; Ellwood and Ledbetter, 1977). Textural variation of the silt fraction is assumed to be a useful indicator of bottom current intensity fluctuation, averaged over a long period $\left(\mathrm{n} \times 10^{2}\right.$ to $\left.\mathrm{n} \times 10^{3} \mathrm{yr}\right)$.

To distinguish between possible hydrodynamic and lithological control of the texture, the average of the median from bulk and decalcified samples, over given stratigraphic intervals was calculated. The correlation coefficient

$$
\begin{aligned}
& {\left[-1<\mathrm{r}<+1 ; \mathrm{r}_{\mathrm{xy}}=\frac{\sigma \mathrm{xy}}{\sigma \mathrm{x} \sigma \mathrm{y}} ; \sigma \mathrm{x}=\sqrt{\frac{\Sigma \mathrm{x}_{\mathrm{i}}^{2}-\mathrm{nx}^{-2}}{\mathrm{n}-1}} ;\right.} \\
& \left.\sigma \mathrm{xy}=\frac{1}{\mathrm{n}-1}\left(\Sigma \mathrm{x}_{\mathrm{i}} \mathrm{y}_{\mathrm{i}}-\frac{1}{\mathrm{n}} \Sigma \mathrm{xi} \Sigma \mathrm{yi}\right)\right]
\end{aligned}
$$

between these two values was then determined. Simultaneous increase of the median from the bulk and decalcified silt fractions of the sediment is, according to our hypothesis, suggestive of an increasing intensity of the bottom current. Increasing value of the correlation coefficient is also indicative of the pre-eminence of dynamic control on the particle size distribution, the influence of the lithology and/or dissolution action being subordinate.

The $25-\mu \mathrm{m}$ median value is situated approximately at mid-point between 1.6 and $57 \mu \mathrm{m}$ on the logarithmic scale. It corresponds thus to Rivière's (1952) "logarithmic facies." Lower median values would indicate deposition through lack of competency, whereas higher median values suggest that the finer silt grade had either never been deposited or had been later eroded. An important criticism can be made to this approach, insofar as sedimentary processes of transport and sedimentation are concerned, which is that the particle dimension of equivalent settling velocity should be considered. It has been already shown 
TABLE 1

Components of the Coarse Fraction

\begin{tabular}{|c|c|c|c|c|}
\hline Terrigenous Components & Authigenic Components & $\begin{array}{l}\text { Biogenic Calcareous } \\
\text { Components }\end{array}$ & $\begin{array}{l}\text { Biogenic Siliceous } \\
\text { Components }\end{array}$ & $\begin{array}{l}\text { Miscellaneous Biogenic } \\
\text { Components }\end{array}$ \\
\hline $\begin{array}{l}\text { Angular quartz } \\
\text { Rounded quartz } \\
\text { Reddish quartz } \\
\text { Mica } \\
\text { Other minerals } \\
\text { Rock fragments } \\
\text { Muddy aggregates }\end{array}$ & $\begin{array}{l}\text { Glauconite } \\
\text { Pyrite } \\
\text { Manganiferous oxide }\end{array}$ & $\begin{array}{l}\text { (Plankton) } \\
\text { Foraminifers, ptero- } \\
\text { pods, ostracodes } \\
\text { (benthos) } \\
\text { Foraminifers } \\
\text { Echinoids } \\
\text { Ostracodes } \\
\text { Bryozoa } \\
\text { Gastropods } \\
\text { Corals } \\
\text { Bivalves } \\
\text { Unspecified carbonates } \\
\text { Miscellaneous }\end{array}$ & $\begin{array}{l}\text { Radiolarians } \\
\text { Diatoms } \\
\text { Sponge spicules }\end{array}$ & $\begin{array}{l}\text { Plants } \\
\text { Fish } \\
\text { Others }\end{array}$ \\
\hline
\end{tabular}

(Berthois and Auffret, 1965) that settling velocities of quartz and mica of the same volume diameter in the range of 10 to $40 \mu \mathrm{m}$ differ by a factor between 3 and 7 . Particle size distributions derived from settling velocities would certainly give different mean size parameters, but by taking into account settling velocities, the percentage of the coarser silt should be reduced inasmuch as their settling velocity could be lower than that of the sphere of same volume diameter. Particles of small volume, unless aggregated, would not have higher settling velocities, hence our results, if compared to particle size distribution derived from settling velocities, would lead probably to overestimation of the coarser grade percentage and hence current intensity.

\section{Accuracy of Textural Data}

In order to evaluate the accuracy of the estimated values of the median, 30 measurements were made on two samples, repeating each of the steps of the analytical procedures following wet sieving of the sediment. The first sample gave an average of $21 \mu \mathrm{m}$ and $\sigma_{\mathrm{p}}=2 \mu \mathrm{m}$

$$
\left(\sigma_{\mathrm{p}}=\sqrt{\frac{\Sigma(\mathrm{x}-\mathrm{m})^{2}}{\mathrm{n}-1}}, \mathrm{~s}_{\mathrm{m}}=\frac{\sigma \mathrm{p}}{\sqrt{\mathrm{n}}}\right) ;
$$

the second, an average of $31 \mu \mathrm{m}$ and $\sigma_{\mathrm{p}}=3.9 \mu \mathrm{m}$. Because we performed three measurements on each sample (two for the residual fraction), we estimated that the $\pm 2 \mathrm{sm}$ confidence intervals (95\% probability) are, respectively, about 2 and $5 \mu \mathrm{m}$ in the first case and 3 to $6 \mu \mathrm{m}$ in the second case.

\section{Lithology}

All available lithological data, shipboard carbonate content measurements, X-ray and carbon-carbonate shore-based routine analyses, and our own results $\left(\mathrm{CaCO}_{3}\right.$ content, quartz content from X-ray analysis) have been processed using a Hewlett-Packard computer, allowing us to draw synthetic lithological logs. The lithological composition of our samples was evaluated through the following procedure (Table 2 ).

The curve of the carbonate content is first drawn from which we interpolate the foraminifers, nannofossils, and unspecified carbonate contents curves, using only our results. Results from shipboard smear-slide examination were used only for checking. We interpolated the quartz content in the interval between our results (semiquantitative evaluation with $\mathrm{NaF}$ internal standard) and results from $X$-ray routine analysis (mineralogical results from SNEA-Pau, this volume). The curve for the clay (plus other $<10 \mu \mathrm{m}$ non-carbonate debris) is interpolated from our results. Siliceous biogenic components make up generally the complement to 100 per cent (this value is checked through smear-slide estimation) although a residual (under $5 \%$ ) of unspecified material remained undetermined.

\section{Partial Sedimentation Rates}

Lithologic composition alone does not permit interpretation of the sedimentary sequence in terms of the sedimentation budget (Lisitzin, 1972) and we have used sedimentation rates (see Site Chapters, this volume) to estimate partial rates for each component.

\section{SITE 402 (Figures 2 and 3, Tables 3A, 3B, 3C, 6, Appendix A)}

Two sub-units of Cenozoic to Quaternary age are present: Sub-unit 1B (175-89.5 m) consisting of upper Eocene marly nannofossil chalk to siliceous marly nannofossil chalk, and Sub-unit 1A (89.5 m to sea bottom), which is upper (?) Pleistocene marly foraminifer nannofossil ooze and calcareous mud.

\section{Sub-Unit 1B (Hole 402, Core 5; Hole 402A, Cores 1 to 4)}

The sediment of Sub-unit 1B accumulated at the rate of $1.2 \mathrm{~cm} / 1000 \mathrm{yr}$. From bottom to top, carbonate accumulation rates increased with a slight concomitant decrease of the siliceous and terrigenous components.

The median of the bulk silt fraction of the upper Eocene samples ranges from 21 to $24 \mu \mathrm{m}$. Medians of the decalcified silt fraction are also low, averaging $18 \mu \mathrm{m}$. The lowermost upper Eocene is characterized by higher median values. This probably relates to a dynamic control, but the low value of the correlation coefficient $(0.06)$ suggests that a dominantly weak bottom current regime prevailed during the late Eocene at Site 402.

The composition of the coarse fraction (Figure 3 ) of the upper Eocene samples indicates that most of the carbonate 
TABLE 2

Analytical Procedures for Lithological Study

\begin{tabular}{ll}
\hline \multicolumn{1}{c}{ Percentage } & \multicolumn{1}{c}{ Methods } \\
\hline $\begin{array}{l}\text { Foraminifers } \\
\text { Nannofossils }\end{array}$ & $\begin{array}{l}\text { Smear slide (visual estimate) } \\
\text { Smear slide (visual examination) checked by the } \\
\text { percentage of finer than } 10 \mu \mathrm{m} \text { carbonate component } \\
\text { evaluated with the Coulter counter } \\
\text { Carbonate content minus (foraminifers + nannofossils) }\end{array}$ \\
$\begin{array}{l}\text { Unspecified } \\
\text { carbonate }\end{array}$ & $\begin{array}{l}\text { X-ray diffraction }-\mathrm{NaF} \text { internal standard } \\
\text { \% finer than } 10 \mu \mathrm{m} \text { of the decalcified sample evalua- } \\
\text { ted with Coulter counter }\end{array}$ \\
$\begin{array}{l}\text { Siliceous } \\
\text { organic }\end{array}$ & \begin{tabular}{l} 
Smear slide and $(100 \%-\Sigma$ other components) \\
\hline
\end{tabular}
\end{tabular}

component derived from in-situ pelagic sedimentation, but the abundant siliceous fraction is essentially of a benthic origin (except for the lowermost part of the section where radiolarians are abundant). The values of planktonic and benthic foraminifers versus unspecified carbonates ratio decrease in the upper part of the section. Because the foraminifer frequency does not change, it implies that the frequency of unspecified carbonates increased at the expense of planktonic foraminifers, thus pointing to possible dissolution.

Two intervals are noteworthy. The bottom of the sub-unit (Cores 4 and 3 ) is characterized by a relatively active bottom current regime, abundant interbedded pebbles of

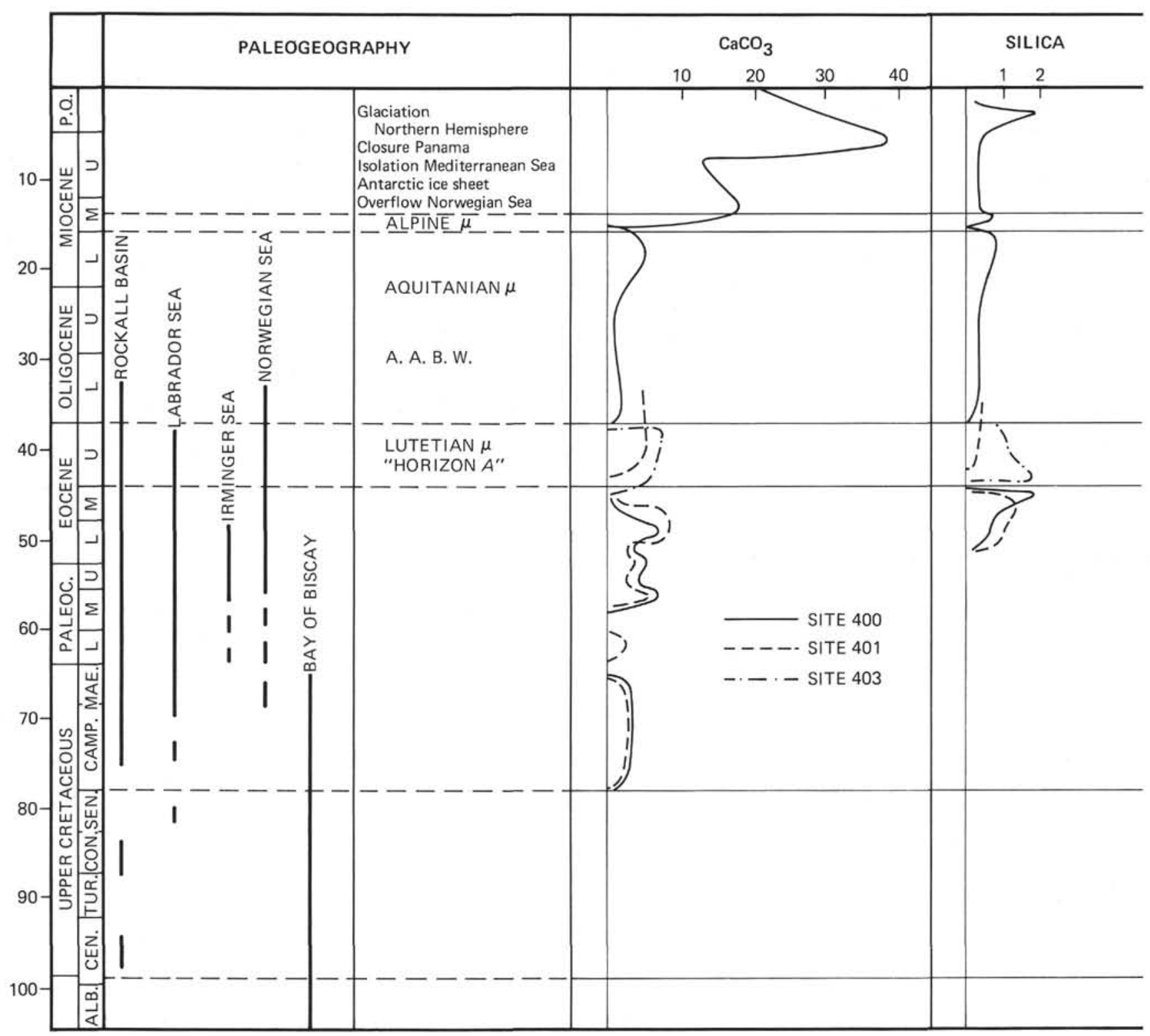

Figure 2. Stratigraphic evolution: sea-floor spreading episodes in different areas of the North Atlantic have been indicated by a continuous line while the rifting episode is figured by dotted line. Partial sedimentation rates $(\mathrm{mm} / 1000 \mathrm{yr})$ : continuous line: Site 400. Dotted line: Site 401. Points and dots: Site 402; sea-level fluctuation after Güssov (1976). Level O corresponds to the average depth of the present-day shelf break. 
shallow water origin, abundant radiolarians in the coarse fraction, and reduced dissolution of the planktonic foraminifers, all of which suggest deposition in a relatively shallow area. At the top of the sub-unit the opposite is observed. Therein are laminations (that could be the consequence of less biological reworking), lower medians of the decalcified silt fractions, fewer radiolarians, and traces of dissolution on the planktonic foraminifers. These observations point to an increase in the water depth.

Shipboard studies produced two interpretations of the paleoenvironment based on benthic foraminifer assemblages. The first assumes that the shallow water assemblage is autochthonous, and a deposition depth ranging from 200 to 500 meters. The second assumes that the fauna was reworked, leading to a water depth ranging from 1000 to 1500 meters. Nannofossil assemblages indicate a neritic environment, although they may also have been displaced into deep water. Because a water depth of 200 to 500 meters would probably have been amenable to other organisms, such as mollusks, bryozoans, etc., and because these are almost absent, we favor a water depth of at least 500 to 1000 meters during the earliest late Eocene.

Debrabant et al. (this volume) point to the singularity of Site 402 relative to Sites 401 and 400 in that the mineralogical assemblage at Site 402 has rare zeolites and fibrous clay and, geochemically, there is less manganese.

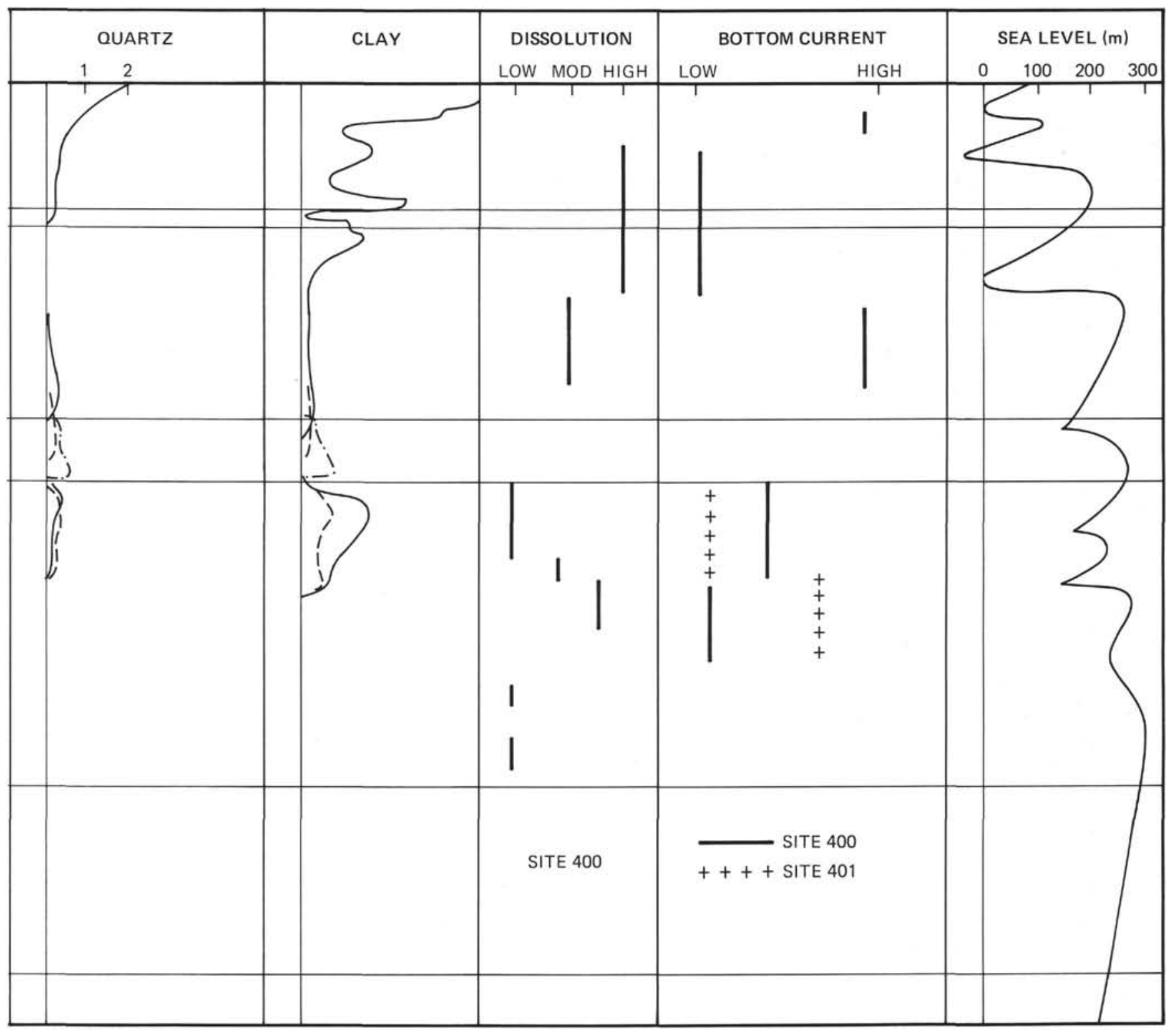

Figure 2. (Continued). 


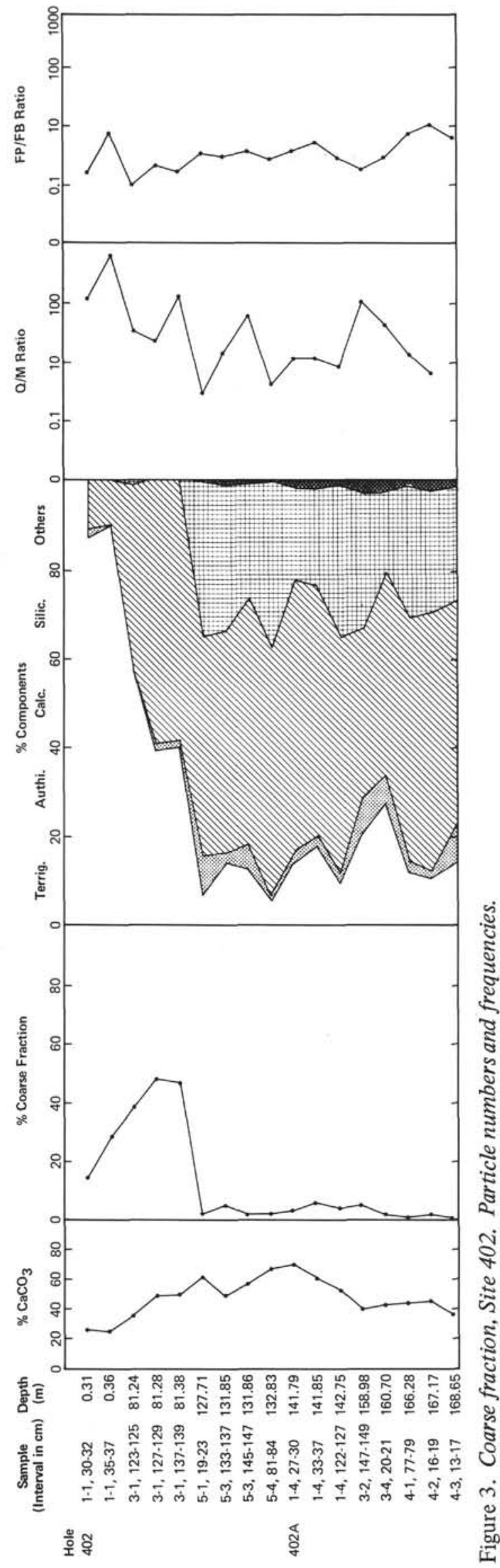

The slight increase in manganese in the uppermost Eocene portion of the section is in good agreement with a deepening of the site at that time. However, according to Ducasse and Peypouquet (this volume) the ostracode assemblages indicate a constant water depth on the order of 2000 meters. Our conclusions are that the evolution of the sedimentation regime at Site 402 during the late Eocene was the following:

1) during earliest Eocene, deposition of highly terrigenous sediment (marked influence of nearby emerged land masses) in a high productivity environment took place in water depths of approximately 1000 to 1500 meters;

2) in late Eocene less terrigenous sediment was deposited in substantially deeper waters, wherein there was increasing calcium carbonate dissolution and more manganese.

\section{Sub-Unit 1A}

Sediment of Sub-unit 1A is dominantly upper Pleistocene homogeneous calcareous mud or marly ooze of grayish olive $(10$ Y $4 / 2)$ to light olive-gray $(5$ Y $5 / 2)$. Sedimentary structures include graded beds (Sample 2-1, 70-100 cm), terrigenous-rich gritty laminations associated with graded bedding and color changes (Sample 3-1, 100-150 cm, Plate 1). Manganese-coated ice-rafted gravels are present throughout.

These sediments were deposited in a canyon, where active reworking probably took place. Although poor core recovery does not permit conclusions to be drawn on the change that may have altered the sedimentary regime during the course of the Quaternary, a detailed study of some of the gritty layers present at the top of the unit was made. Samples 402-1-1, 30-32 cm and 402-1-1, 35-37 cm belong to an interval of gritty laminae, above a possible slumped bed (Sample 1-1, 40-80 cm). The upper one, sampled immediately above a gritty lamina, is a grayish olive (10 Y 4/2) homogeneous calcareous mud. The lower is a light olive-gray (5Y $5 / 2)$ calcareous mud, belonging to a gritty layer that is characterized by sharp upper and lower boundaries. The composition of its coarse fraction indicates simultaneous occurrence of pelagic foraminifers and terrigenous component (iron-coated quartz). The median of the silt fractions are, respectively, 33 and $32 \mu \mathrm{m}$ for the bulk sample and 25 and $30 \mu \mathrm{m}$ for the decalcified sediment. These values indicate possible hydrodynamic sorting. It appears also that the coarser levels result from a concentration of coarse non-carbonate debris, either through non-deposition or erosion of the clay and fine silt grade. It is thus probable that bottom currents, perhaps of tidal origin, were involved in the genesis of this coarser gritty lamina.

SITE 401 (Figure 2, Tables 4A, 4B, 4C, 6, Appendix B)

\section{Unit 3}

\section{Sub-Unit 3C and Sub-Unit 3B (Cores 18 to 13, lower portion)}

Upper Cretaceous (Campanian and Maestrichtian) to Paleocene calcareous chalk constitute this unit. These sediments overlie a 5-cm-thick layer of Aptian shallow water $(<500 \mathrm{~m})$ chalk. Sample 19-1, 118-120 cm, which contains siderite, was sampled immediately above the chalk. The coarse fraction $(43 \%)$ comprises 5 per cent planktonic foraminifers and 2 per cent benthic foraminifers. Also in this fraction are manganese oxides, pyrite, limonite, 
TABLE 3A

Lithology, Holes 402/402A

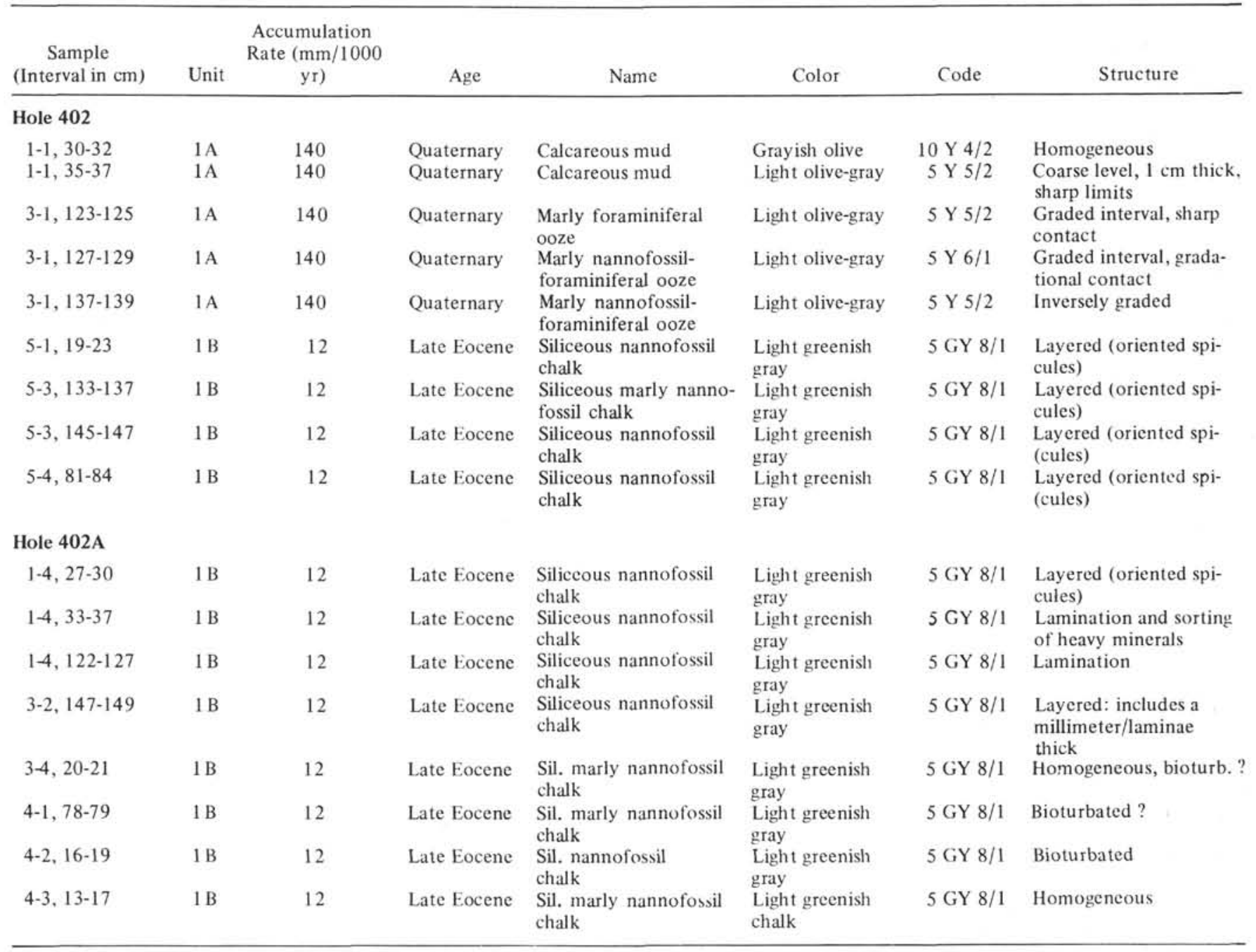

and lithoclasts of older ochrous marly limestones. The medians of the silt fractions are 17 and $19 \mu \mathrm{m}$, respectively, suggesting prevailing low-energy bottom currents. The coarse fraction of the only Maestrichtian sample studied is rich in planktonic foraminifers $(84 \%)$ and contains traces of manganese.

The grayish orange (10 YR 7/4) lower Paleocene nannofossil chalk constitutes an interval 4 meters thick. The sediment is slightly mottled, with evidence of slumped beds; laminations are also present. It is separated from the upper Paleocene by a $2.6-\mathrm{m} . \mathrm{y}$. hiatus. The sedimentation rate has not been estimated, but is probably low. The upper Paleocene grayish orange calcareous chalk displays various sedimentary structures such as zones characterized by laminations or grading, alternating with homogeneous intervals. Microfaulting is also common. The sedimentation rate is low, about $0.5 \mathrm{~cm} / 1000 \mathrm{yr}$.

Sub-unit 3B comprises slightly mottled dark yellowish brown (10 YR 4/2) to grayish orange (10 YR 7/4) upper Paleocene to lower Eocene marly calcareous chalk that accumulated at the rate of $0.5 \mathrm{~cm} / 1000 \mathrm{yr}$. Coarse fraction observations (Figure 4) suggest that the sedimentary regime remained relatively stable during the course of the
Paleocene. They are comprised dominantly planktonic foraminifers (63 to $97 \%$ ). Benthic foraminifers reach only 1.3 per cent, but some ostracodes are present and the terrigenous components increase upwards. The planktonic versus benthic foraminifer ratio remains stable and suggests low dissolution. Two samples are characterized by a siliceous component, comprising radiolarians and sponge spicules.

Average median of the silt fractions for the bulk sediment and the decalcified sediments are $28 \mu \mathrm{m}$ and $19 \mu \mathrm{m}$, respectively. The correlation coefficient $(0.26)$ points to slightly hydrodynamical sorting by bottom currents.

\section{Sub-Unit 3A (Core 13 upper portion to Core 11, Section 2)}

Lower Eocene light brown to grayish orange nannofossil chalk and marly nannofossil chalk dominate the section. The sedimentation rate $(0.5 \mathrm{~cm} / 1000 \mathrm{yr})$ is identical to that of the upper Paleocene. Mottling is moderate and the section is characterized by numerous slickensided fractures and inclined contacts. Noteworthy is the occurrence of a collophane layer at the base of the unit (Plate 2).

Coarse fraction contents are relatively high and range from 2 to 15 per cent. It comprises about 80 per cent 
TABLE 3B

Lithology, Holes 402/402A

\begin{tabular}{|c|c|c|c|c|c|c|c|c|c|c|c|c|c|}
\hline $\begin{array}{c}\text { Sample } \\
\text { (Interval in } \mathrm{cm} \text { ) }\end{array}$ & $\mathrm{CaCO}_{3}$ & Foram. & Nanno. & Unsp. & Quartz & $\begin{array}{l}\text { Clay, } \\
\text { Other }\end{array}$ & Silica & $\begin{array}{c}\mathrm{Md} \\
(\mu \mathrm{m})\end{array}$ & $\begin{array}{c}\text { So } \\
\text { (bulk) }\end{array}$ & $\begin{array}{c}\text { Sk } \\
\text { (bulk) }\end{array}$ & $\begin{array}{c}1 \% \\
(\mu \mathrm{m})\end{array}$ & $\begin{array}{c}\text { Md }(\mu \mathrm{m}) \\
\text { Silt } \\
\text { (bulk) }\end{array}$ & $\begin{array}{c}\text { Md }(\mu \mathrm{m}) \\
\text { Silt } \\
\text { (residual) }\end{array}$ \\
\hline \multicolumn{14}{|l|}{ Hole 402} \\
\hline $\begin{array}{l}1-1,30-32 \\
1-1,35-37 \\
3-1,123-125 \\
3-1,127-129 \\
3-1,137-139 \\
5-1,19-23 \\
5-3,133-137 \\
5-3,145-147 \\
5-4,81-84\end{array}$ & $\begin{array}{l}25 \\
24 \\
35 \\
48 \\
49 \\
61 \\
48 \\
55 \\
67\end{array}$ & $\begin{array}{r}2 \\
3 \\
20 \\
25 \\
21\end{array}$ & $\begin{array}{r}14 \\
16 \\
7 \\
10 \\
11 \\
24 \\
19 \\
23 \\
30\end{array}$ & $\begin{array}{r}9 \\
5 \\
8 \\
13 \\
17 \\
37 \\
29 \\
32 \\
37\end{array}$ & $\begin{array}{r}62 \\
58 \\
43 \\
29 \\
29 \\
10 \\
12 \\
6 \\
5\end{array}$ & $\begin{array}{l}10 \\
17 \\
21 \\
23 \\
32 \\
15 \\
22 \\
19 \\
10\end{array}$ & $\begin{array}{l}14 \\
18 \\
20 \\
18\end{array}$ & $\begin{array}{l}25 \\
40 \\
60 \\
60 \\
60 \\
16 \\
16 \\
13 \\
16\end{array}$ & $\begin{array}{l}2.45 \\
2.74 \\
1.94 \\
\\
3.08 \\
2.31 \\
2.31 \\
1.83 \\
1.77\end{array}$ & $\begin{array}{l}0.96 \\
0.73 \\
0.65 \\
\\
1.06 \\
0.75 \\
0.75 \\
0.77 \\
0.78\end{array}$ & $\begin{array}{r}160 \\
450 \\
400 \\
\\
420 \\
70 \\
1000 \\
90 \\
70\end{array}$ & $\begin{array}{l}33 \\
32 \\
41 \\
29 \\
28 \\
23 \\
23 \\
22\end{array}$ & $\begin{array}{l}25 \\
\\
26 \\
24 \\
23 \\
18 \\
18 \\
18\end{array}$ \\
\hline \multicolumn{14}{|l|}{ Hole 402A } \\
\hline $\begin{array}{l}1-4,27-30 \\
1-4,33-37 \\
1-4,122-127 \\
3-2,147-149 \\
3-4,20-21 \\
4-1,78-79 \\
4-2,16-19 \\
4-3,13-17\end{array}$ & $\begin{array}{l}70 \\
61 \\
53 \\
40 \\
43 \\
44 \\
45 \\
36\end{array}$ & & $\begin{array}{l}30 \\
26 \\
23 \\
10 \\
11 \\
20 \\
20 \\
19\end{array}$ & $\begin{array}{l}40 \\
35 \\
30 \\
30 \\
32 \\
24 \\
25 \\
17\end{array}$ & $\begin{array}{r}6 \\
6 \\
8 \\
11 \\
10 \\
10\end{array}$ & $\begin{array}{l}12 \\
18 \\
17 \\
30 \\
24 \\
23 \\
23 \\
26\end{array}$ & $\begin{array}{l}12 \\
15 \\
22 \\
19 \\
23 \\
23 \\
32 \\
29\end{array}$ & $\begin{array}{l}13 \\
16 \\
16 \\
16 \\
16 \\
13 \\
13 \\
13\end{array}$ & $\begin{array}{l}2.04 \\
2.31 \\
2.31 \\
2.00 \\
1.77 \\
2.04 \\
2.04 \\
2.04\end{array}$ & $\begin{array}{l}0.96 \\
0.75 \\
0.75 \\
1.00 \\
0.78 \\
0.96 \\
0.96 \\
0.96\end{array}$ & $\begin{array}{r}230 \\
450 \\
260 \\
500 \\
70 \\
60 \\
70 \\
56\end{array}$ & $\begin{array}{l}22 \\
22 \\
24 \\
21 \\
22 \\
22 \\
21 \\
23\end{array}$ & $\begin{array}{l}18 \\
18 \\
18 \\
17 \\
17 \\
20 \\
19 \\
19\end{array}$ \\
\hline
\end{tabular}

planktonic foraminifers, and up to 31 per cent radiolarians in Sample 11-4, 25-31 cm; siliceous components are lacking in other samples.

The average of the silt medians for bulk samples is almost identical to those of Unit $3(\mathrm{~B}+\mathrm{C})$, but the dispersion is less $(\sigma \mathrm{x}=2.8$ against $5 \mu \mathrm{m})$ The average decalcified silt median $(20 \mu \mathrm{m})$ is also close to the preceding unit, but is more dispersed $(\sigma=3.1$ against $1.7 \mu \mathrm{m})$. Correlation between median of both fractions $(\gamma=-0.02)$ is poor.

These observations indicate that deposition of the lower Eocene sediment occurred under a relatively weak bottom current regime and a moderate dissolution rate.

\section{Unit 2}

\section{Sub-Unit 2B (Core 11, Section 1 to Core 5)}

This section comprises middle Eocene pale olive (10 Y $6 / 2$ ) to greenish gray (5 GY 6/1) nannofossil chalk that accumulated at the rate of $1.3 \mathrm{~cm} / 1000 \mathrm{yr}$. They are separated from the upper Eocene by a 1.8-m.y. hiatus. The sediments of Cores 10 and 9 are devoid of sedimentary structures with the exceptions of few Zoophycos burrows. Core 8 is both laminated and burrowed. Core 7 is laminated and sediments of Cores 6 and 5 are moderately to intensely burrowed (Plate 2).

Coarse fraction contents are higher at the beginning and at the end of the middle Eocene ( 5 to $10 \%$ ); they average 2 per cent through the middle Eocene. The carbonate fraction is dominant, with the exception of Samples 9-2, 137-140 $\mathrm{cm}$ and $8-3,106-109 \mathrm{~cm}$, where the siliceous component is more abundant. The carbonate comprises dominantly planktonic foraminifers, the $\mathrm{PF} / \mathrm{BF}$ ratio ranges being between 20 and 50; benthic foraminifers range between 1 and 3 per cent, hence variations of the $\mathrm{PF} / \mathrm{BF}$ ratio are related to fluctuations in the abundance of the planktonic foraminifers which may be related to dissolution; this appears to have been less pronounced by the end of the middle Eocene where the siliceous component ranges from 12 to 49 per cent. Radiolarians (sometimes badly preserved) are abundant ranging from 23 to 43 per cent; sponge spicules reach up to 5 per cent. Pyrite is abundant in Core 9; fish debris although rare is always present, amounting to 1 per cent in Sample 10-7, 16-18 cm. The general impression that results from the examination of the coarse fraction is that deposition of these sediments occurred in a context of high pelagic productivity. Average median of the bulk and decalcified silt are almost identical to those of Unit 3, but the medians of the decalcified silt are less dispersed and there is also a slight increase $(0.18$ against -0.02$)$ of the correlation coefficient.

These observations indicate that the sedimentation rate increase that occurred at the lower-middle Eocene boundary resulted from increasing terrigenous supply and decreasing dissolution rates. The evolution occurred within a predominantly weak bottom current regime and the middle-upper Eocene hiatus at this site does not appear to have been preceded by increasing bottom current or dissolution rates.

\section{Sub-Unit 2A (Cores 4 to 2)}

The sub-unit consists of upper Eocene and lower Oligocene light greenish gray (5 GY 8/1) nannofossil chalk, that accumulated at the approximate rate of $0.7 \mathrm{~cm} / 1000 \mathrm{yr}$. The coarse fraction ( 2 to $7 \%$ ) is composed mostly of planktonic foraminifers $(75 \%)$ and radiolarians $(8 \%)$. The medians of the bulk silt fraction are high $(31$ and $28 \mu \mathrm{m})$ but those of the decalcified samples are low (19 and $18 \mu \mathrm{m})$. In view of the few samples available no hypothesis about the environmental conditions can be deduced. 
TABLE 3C

Lithology, Holes 402/402A

\begin{tabular}{|c|c|c|c|c|c|c|c|c|c|c|c|c|c|c|c|c|c|c|c|c|c|c|c|c|}
\hline $\begin{array}{c}\text { Sample } \\
\text { (Interval in } \mathrm{cm} \text { ) }\end{array}$ & QR & QRO QA & MIC OMIN & ROC & MUDA & PYR & GLAU & MAN & PFO & PTER & BFO & ECH & oST & LAM & BRY & $\mathrm{COR}$ & UNSP & RAD & DIA & SPIC & PLA & FISH & отн & TOTAL \\
\hline \multicolumn{25}{|c|}{ Number of Partickes } \\
\hline \multicolumn{25}{|l|}{ Hole 402} \\
\hline $1-1,30-32$ & 23 & 446 & 4 & 1 & 3 & 4 & 5 & 2 & 13 & 0 & 8 & 0 & 0 & 0 & 0 & 0 & 41 & 0 & 0 & 0 & 0 & 0 & 0 & 577 \\
\hline 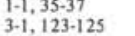 & ${ }_{25}^{44}$ & $\begin{array}{l}544 \\
524\end{array}$ & $\begin{array}{c}1 \\
17\end{array}$ & ${ }_{6}^{2}$ & $\frac{0}{2}$ & $\frac{2}{5}$ & ${ }_{0}^{4}$ & $\begin{array}{l}0 \\
0\end{array}$ & $\begin{array}{r}37 \\
161\end{array}$ & $\stackrel{0}{0}$ & $\begin{array}{r}5 \\
165\end{array}$ & ${ }_{3}^{0}$ & $\begin{array}{l}0 \\
0\end{array}$ & $\stackrel{0}{0}$ & ${ }_{0}^{0}$ & ${ }_{0}^{0}$ & $\begin{array}{r}25 \\
106\end{array}$ & i & 1 & $\begin{array}{l}0 \\
3\end{array}$ & ${ }_{0}^{0}$ & ${ }_{0}^{0}$ & ${ }_{0}^{0}$ & $\begin{array}{r}701 \\
1052\end{array}$ \\
\hline $3-1,127-129$ & 17 & 341 & 17 & 13 & $\frac{2}{2}$ & 7 & 7 & ${ }_{0}^{0}$ & $\begin{array}{l}161 \\
342\end{array}$ & $0_{0}^{0}$ & $\begin{array}{l}163 \\
156\end{array}$ & $0_{0}^{3}$ & $0_{0}^{0}$ & ${ }_{0}^{0}$ & 0 & ${ }_{0}^{0}$ & $\begin{array}{l}106 \\
127\end{array}$ & 0 & ${ }_{0}^{1}$ & 0 & $0_{0}^{0}$ & $0_{0}^{0}$ & ${ }_{0}^{0}$ & $\begin{array}{l}1052 \\
1050\end{array}$ \\
\hline $3-1,137-139$ & 13 & 349 & 3 & 13 & 0 & 3 & 9 & ${ }_{0}^{0}$ & 283 & 0 & 172 & 6 & 0 & 0 & 0 & 0 & 129 & 0 & ${ }_{0}^{0}$ & 0 & 0 & 0 & 0 & $\begin{array}{l}1050 \\
1003\end{array}$ \\
\hline $5-1,19 \cdot 23$ & 5 & 31 & 13 & 4 & i & 70 & 2 & 0 & 186 & 0 & 55 & i & 0 & 0 & 0 & 0 & 167 & 16 & $s$ & 266 & 1 & 0 & $0_{0}$ & 823 \\
\hline $5-3,133-137$ & 1 & 61 & 4 & 21 & 25 & 10 & 9 & 0 & 158 & 0 & 55 & i & 0 & 0 & 0 & 0 & 208 & 39 & 0 & 244 & 0 & 0 & 0 & 840 \\
\hline $\begin{array}{l}5 \cdot 3,145-147 \\
54.81-84\end{array}$ & i & 56 & $\frac{1}{5}$ & 9 & 6 & 27 & 13 & 0 & 183 & 0 & S1 & 1 & 1 & 0 & 1 & 0 & 140 & 32 & 0 & 141 & 2 & 2 & 0 & 677 \\
\hline $54,81-84$ & & 17 & 5 & 0 & & & & & 149 & & 57 & & 0 & & 0 & 0 & 135 & 49 & 0 & 181 & & 0 & & 612 \\
\hline \multicolumn{25}{|l|}{ Hole $402 \mathrm{~A}$} \\
\hline $14,27 \cdot 30$ & 1 & 44 & 8 & 0 & 42 & 12 & 6 & 0 & 214 & 0 & 59 & 5 & 0 & 0 & 0 & 0 & 163 & 23 & 2 & 125 & 0 & 1 & 8 & 718 \\
\hline $\begin{array}{l}14.33-37 \\
14.122 .127\end{array}$ & $\begin{array}{l}0 \\
0\end{array}$ & 44 & 3 & 0 & ${ }_{26}^{46}$ & 9 & 5 & 0 & 178 & 0 & 35 & 3 & 1 & 0 & 0 & 0 & 96 & 18 & 4 & 102 & 0 & 0 & 9 & 559 \\
\hline $\begin{array}{l}1-4,1222-127 \\
3 \cdot 2,147-149\end{array}$ & $\begin{array}{l}0 \\
1\end{array}$ & $\begin{array}{r}16 \\
101\end{array}$ & $\begin{array}{l}3 \\
6 \\
\end{array}$ & ${ }_{2}^{0}$ & ${ }_{12}^{26}$ & $\begin{array}{r}8 \\
33\end{array}$ & $\begin{array}{r}4 \\
15\end{array}$ & $\stackrel{0}{0}$ & $\begin{array}{l}116 \\
80\end{array}$ & ${ }_{0}^{2}$ & $\begin{array}{l}44 \\
47\end{array}$ & $\frac{1}{2}$ & $\stackrel{0}{0}$ & $\stackrel{0}{0}$ & $\stackrel{0}{0}$ & $\stackrel{0}{0}$ & $\begin{array}{l}101 \\
100\end{array}$ & 14 & 1 & 158 & 0 & 0 & $3_{17}^{3}$ & 499 \\
\hline $34,20-21$ & 1 & 80 & 2 & 1 & 61 & 15 & $\begin{array}{l}15 \\
19\end{array}$ & ${ }_{0}^{0}$ & $\begin{array}{l}80 \\
95\end{array}$ & $\begin{array}{l}0 \\
0\end{array}$ & 35 & $i_{1}^{2}$ & $\begin{array}{l}0 \\
0\end{array}$ & $\begin{array}{l}0 \\
0\end{array}$ & $\begin{array}{l}0 \\
0\end{array}$ & ${ }_{0}^{0}$ & $\begin{array}{l}100 \\
116\end{array}$ & $\begin{array}{l}35 \\
34\end{array}$ & $\frac{1}{2}$ & $\begin{array}{r}146 \\
61\end{array}$ & $\begin{array}{l}0 \\
0 \\
0\end{array}$ & $\begin{array}{l}0 \\
0\end{array}$ & $\begin{array}{l}17 \\
12\end{array}$ & $\begin{array}{l}600 \\
536\end{array}$ \\
\hline $4-1,77-79$ & 0 & 51 & 4 & 2 & 24 & 14 & 3 & 0 & 280 & 0 & 41 & 2 & 0 & 0 & 0 & 0 & 62 & 69 & 8 & 135 & 0 & 1 & 4 & 701 \\
\hline 4-2, 16-19 & ${ }_{0}^{0}$ & 12 & 2 & 0 & 29 & 8 & 0 & 0 & 185 & 0 & $\begin{array}{l}19 \\
33\end{array}$ & 0 & 1 & 0 & 0 & 0 & 43 & 35 & $?$ & 75 & 0 & 0 & 9 & 426 \\
\hline 4.3,13.17 & & & & & 38 & 40 & 8 & & 189 & 0 & 33 & 1 & 0 & 0 & 0 & 0 & 54 & 75 & 2 & 63 & & & 6 & 545 \\
\hline \multicolumn{25}{|c|}{ Particles Frequency } \\
\hline \multicolumn{25}{|l|}{ Hole 402} \\
\hline $1-1,30-32$ & 4.0 & $\begin{array}{ll}4.0 & 77.3\end{array}$ & 0.7 & 0.2 & 0.5 & 0.7 & 0.9 & 0.3 & 2.3 & 0.0 & 1.4 & 0.0 & 0.0 & 0.0 & 0.0 & 0.0 & 7.1 & 0.0 & 0.0 & 0.0 & 0.0 & 0.0 & 0.0 & \\
\hline $\begin{array}{l}1-1,35-37 \\
37,123-125\end{array}$ & 6.3 & $3.9 \quad 77.6$ & 0.1 & 0.3 & 0.0 & 0.3 & 0.6 & 0.0 & 5.3 & 0.0 & 0.7 & 0.0 & 0.0 & 0.0 & 0.0 & 0.0 & 3.6 & 0.0 & 0.1 & 0.0 & 0.0 & 0.0 & 0.0 & \\
\hline $\begin{array}{l}3-7,123-125 \\
3-1,127+129\end{array}$ & 2.4 & $\begin{array}{ll}2.3 & 49.8 \\
1.3 & 32.5\end{array}$ & 1.6 & 0.6 & 0.2 & 0.5 & 0.0 & 0.0 & 15.3 & ${ }^{0.0}$ & 15.7 & 0.3 & 0.0 & 0.0 & 0.0 & 0.0 & 10.1 & 0.1 & 0.1 & 0.3 & 0.0 & 0.0 & 0.0 & \\
\hline $3-1,137-139$ & 1.6 & $\begin{array}{l}32.3 \\
3.48\end{array}$ & 1.6 & 1.2 & 0.2 & 0.7 & 0.7 & 0.0 & 32.6 & 0.0 & 14.9 & 0.0 & 0.0 & 0.0 & 0.0 & 0.0 & 12.1 & 0.0 & 0.0 & 0.0 & 0.0 & 0.0 & 0.0 & \\
\hline $5-1,19 \cdot 23$ & 0.3 & $\begin{aligned} 34.8 \\
3.8\end{aligned}$ & 0.3 & 1.3 & 0.0 & 0.3 & 0.9 & 0.0 & 28.2 & $0_{00}^{0.0}$ & 17.1 & 0.6 & 0.0 & 0.0 & 0.0 & 0.0 & $\begin{array}{l}12.9 \\
203\end{array}$ & 0.0 & 0.0 & 0.0 & ${ }_{0.0}^{0.0}$ & 0.0 & 0.0 & \\
\hline $5 \cdot 3,133 \cdot 137$ & $\begin{array}{l}0.1 \\
0.1\end{array}$ & 7.3 & $\begin{array}{l}1.6 \\
0.5\end{array}$ & ${ }_{2.5}^{0.5}$ & $\begin{array}{l}0.1 \\
3,0\end{array}$ & $\begin{array}{l}8.5 \\
1.2\end{array}$ & 0.2 & ${ }_{0.0}^{0.0}$ & $\begin{array}{l}21.6 \\
18.8\end{array}$ & $\begin{array}{l}0.0 \\
0.0\end{array}$ & $\begin{array}{l}6.7 \\
6.5\end{array}$ & 0.1 & 0.0 & $\begin{array}{l}0.0 \\
0.0\end{array}$ & $\begin{array}{l}0.0 \\
0.0\end{array}$ & $\begin{array}{l}0.0 \\
0.0\end{array}$ & $\begin{array}{l}20.3 \\
24.8\end{array}$ & $\begin{array}{l}1.9 \\
4.6\end{array}$ & 00 & 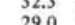 & 0.1 & 00 & 00 & \\
\hline & 0.1 & 8.3 & 01 & 3 & 3.0 & 1.2 & 1.1 & 0.0 & 10.6 & 0.0 & 年 & . & 0.0 & 0.0 & 促 & 0 & 24.8 & $\begin{array}{lll}4.6 & \end{array}$ & 00 & 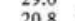 & 0.03 & 0.03 & 0.0 & \\
\hline $5-4,81-84$ & 0.2 & 2.8 & $\begin{array}{l}0.1 \\
0.8\end{array}$ & $\begin{array}{l}1.3 \\
0.0\end{array}$ & $\begin{array}{l}0.9 \\
1.3\end{array}$ & $\begin{array}{l}4.0 \\
0.7\end{array}$ & 0.5 & $\begin{array}{l}0.0 \\
0.0\end{array}$ & $\begin{array}{l}27.0 \\
24.3\end{array}$ & $\begin{array}{l}0.0 \\
0.0\end{array}$ & 9.3 & $\begin{array}{l}0.1 \\
0.2\end{array}$ & $\begin{array}{l}0.1 \\
0.0\end{array}$ & $\begin{array}{l}0.0 \\
0.0\end{array}$ & $\begin{array}{l}0.1 \\
0.0\end{array}$ & $\begin{array}{l}0.0 \\
0.0\end{array}$ & $\begin{array}{l}20.7 \\
22.1\end{array}$ & $\begin{array}{l}4.7 \\
8.0\end{array}$ & $\begin{array}{l}0.0 \\
0.0\end{array}$ & $\begin{array}{l}20.8 \\
29.6\end{array}$ & $\begin{array}{l}0.3 \\
0.0\end{array}$ & $\begin{array}{l}0.3 \\
0.0\end{array}$ & 0.0 & \\
\hline \multicolumn{25}{|l|}{ Hole $402 \mathrm{~A}$} \\
\hline $1-4,27-30$ & 0.1 & 6.1 & 0.6 & 0.0 & 5.8 & 1.7 & 0.8 & 0.0 & 29.8 & 0.0 & 8.2 & 0.7 & 0.0 & 0.0 & 0.0 & 0.0 & 22.7 & 3.2 & 0.3 & 17.4 & 0.0 & 0.1 & 1.1 & \\
\hline $1-4,33-37$ & 0.0 & 7.9 & 0.7 & 0.0 & 8.2 & 1.6 & 0.9 & 0.0 & 31.8 & 0.0 & 6.3 & 0.5 & 0.2 & 0.0 & 0.0 & 0.0 & 17.2 & 3.2 & 0.7 & 18.2 & 0.0 & 0.0 & 1.6 & \\
\hline $\begin{array}{l}14,122.127 \\
3-2,147-149\end{array}$ & $\begin{array}{l}0.0 \\
0.2\end{array}$ & $\begin{array}{rr}0.0 & 3.2 \\
0.2 & 16.8\end{array}$ & $\begin{array}{l}0.4 \\
0.2\end{array}$ & $\begin{array}{l}0.0 \\
0.3\end{array}$ & $\begin{array}{l}5.2 \\
20\end{array}$ & 1.6 & 0.8 & 0.0 & 23.2 & 0.4 & 8.8 & 0.2 & 0.0 & 0.0 & 0.0 & 0.0 & 20.2 & 2.8 & 0.2 & 31.7 & 0.0 & 0.0 & 0.6 & \\
\hline $3-4,20-21$ & 0.2 & $\begin{array}{ll}0.2 & 16.8 \\
0.2 & 14.9\end{array}$ & 0.2 & 0.2 & 2.0 & 2.8 & 2.35 & ${ }_{0.0}^{0.0}$ & $\begin{array}{l}13.3 \\
11.7\end{array}$ & 0.0 & 7.8 & 0.3 & 0.0 & 0.0 & 0.0 & 0.0 & 16.7 & $\begin{array}{l}5.8 \\
6.3\end{array}$ & 0.2 & 24.3 & 0.0 & 0.0 & 2.8 & \\
\hline $4-1,77-79$ & 0.0 & 7.3 & 0.6 & 0.3 & 3.4 & 2.0 & 0.4 & 0.0 & 39.9 & ${ }_{0.0}^{0.0}$ & $\begin{array}{l}6.3 \\
5.8\end{array}$ & $\begin{array}{l}0.2 \\
0.3\end{array}$ & $\begin{array}{l}0.0 \\
0.0\end{array}$ & $\begin{array}{l}0.0 \\
0.0\end{array}$ & $\begin{array}{l}0.0 \\
0.0\end{array}$ & $\begin{array}{l}0.0 \\
0.0\end{array}$ & $\begin{array}{r}21.6 \\
8.8\end{array}$ & $\begin{array}{l}6.3 \\
9.8\end{array}$ & 0.4 & $\begin{array}{l}11.4 \\
19.3\end{array}$ & $\begin{array}{l}0.0 \\
0.0\end{array}$ & $\begin{array}{l}0.0 \\
0.1\end{array}$ & $\begin{array}{l}2.2 \\
0.6\end{array}$ & \\
\hline \multirow{3}{*}{ 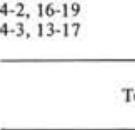 } & 0.0 & 2.8 & 0.5 & 0.0 & 6.8 & 1.9 & 0.0 & 0.0 & 43.4 & 0.0 & 4.5 & 0.0 & 0.2 & 0.0 & 0.0 & 0.0 & $\begin{array}{r}0.0 \\
10.1\end{array}$ & 8.2 & 1.6 & 17.6 & 0.0 & 0.0 & 2.1 & \\
\hline & 0.0 & 6.4 & 0.0 & 0.0 & 7.0 & 7.3 & 1.5 & 0.0 & 34.7 & 0.0 & 6.1 & 0.2 & 0.0 & 0.0 & 0.0 & 0.0 & 9.9 & 13.8 & 0.4 & 11.6 & 0.0 & 0.2 & 1.1 & \\
\hline & $\begin{array}{l}\text { Terrigenous } \\
(\%)\end{array}$ & $\begin{array}{c}\text { Authigenic } \\
(\%)\end{array}$ & $\begin{array}{c}\text { Calcareous } \\
(\%)\end{array}$ & $\begin{array}{c}\text { Siliceous } \\
(\%)\end{array}$ & $\begin{array}{c}\text { Miscellan } \\
(\%)\end{array}$ & ineous & & & & & & & & & & & & & & & & & & \\
\hline \multicolumn{25}{|l|}{ Hole 402} \\
\hline $1-1,30-32$ & 87.35 & 1.91 & 10.75 & 0.00 & 0.00 & & & & & & & & & & & & & & & & & & & \\
\hline $35 \cdot 37$ & 89.44 & 0.86 & 9. & 0.14 & 00 & & & & & & & & & & & & & & & & & & & \\
\hline $\begin{array}{l}3-1,123-125 \\
3-1,127-129\end{array}$ & $\begin{array}{l}57.70 \\
39.14\end{array}$ & 0.48 & $\begin{array}{l}41.35 \\
5950\end{array}$ & 0.48 & 0.00 & & & & & & & & & & & & & & & & & & & \\
\hline $\begin{array}{l}3-1,127-129 \\
3-1,137-139\end{array}$ & $\begin{array}{l}39.14 \\
39.98\end{array}$ & $\begin{array}{l}1.33 \\
1.20\end{array}$ & $\begin{array}{l}59.52 \\
58.82\end{array}$ & $\begin{array}{l}0.00 \\
0.00\end{array}$ & 0.00 & & & & & & & & & & & & & & & & & & & \\
\hline $\begin{array}{l}3-1,19-23 \\
5-139\end{array}$ & $\begin{array}{r}39.98 \\
6.56\end{array}$ & $\begin{array}{l}1.20 \\
8.75\end{array}$ & $\begin{array}{l}58.82 \\
49.70\end{array}$ & $\begin{array}{r}0.00 \\
34.87\end{array}$ & $\begin{array}{l}0.00 \\
0.12\end{array}$ & & & & & & & & & & & & & & & & & & & \\
\hline $5-3,133-137$ & 13.81 & 2.26 & 50.24 & 33.69 & 0.00 & & & & & & & & & & & & & & & & & & & \\
\hline $5-3,145-147$ & 12.26 & 5.91 & 55.54 & 25. & 0.39 & & & & & & & & & & & & & & & & & & & \\
\hline $5-4,81-84$ & 5.39 & 1.14 & 55.88 & 37.58 & 0.00 & & & & & & & & & & & & & & & & & & & \\
\hline \multicolumn{25}{|l|}{ Hole 402A } \\
\hline $14,27-30$ & 13.93 & 2.51 & 61.42 & 20.8 & 1.25 & & & & & & & & & & & & & & & & & & & \\
\hline & $\begin{array}{l}17.711 \\
9.42\end{array}$ & 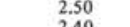 & & & & & & & & & & & & & & & & & & & & & & \\
\hline $\begin{array}{l}14,122-127 \\
3-2,147-149\end{array}$ & $\begin{array}{r}9.42 \\
20.67\end{array}$ & $\begin{array}{l}2.40 \\
8.00\end{array}$ & $\begin{array}{l}52 . \\
38\end{array}$ & 34 & 0 . & & & & & & & & & & & & & & & & & & & \\
\hline $3-4,20-21$ & 27.24 & 6.34 & $\begin{array}{l}30.08 \\
46.08\end{array}$ & $\begin{array}{l}30.33 \\
18.10\end{array}$ & $\begin{array}{l}2.83 \\
2.24\end{array}$ & & & & & & & & & & & & & & & & & & & \\
\hline $4-1,77-79$ & 11.70 & 2.43 & 54 & 30.24 & 0.71 & & & & & & & & & & & & & & & & & & & \\
\hline $\begin{array}{l}4-2,16-19 \\
4-3,13 \cdot 17\end{array}$ & $\begin{array}{l}10.33 \\
13.39\end{array}$ & $\begin{array}{l}1.88 \\
8.81\end{array}$ & $\begin{array}{l}58.22 \\
50.83\end{array}$ & $\begin{array}{l}27.46 \\
25.69\end{array}$ & $\begin{array}{l}2.11 \\
1.28\end{array}$ & & & & & & & & & & & & & & & & & & & \\
\hline & & & & & & & & & & & & & & & & & & & & & & & & \\
\hline
\end{tabular}


TABLE 4A

Lithology, Site 401

\begin{tabular}{|c|c|c|c|c|c|c|c|}
\hline $\begin{array}{c}\text { Sample } \\
(\text { Interval in } \mathrm{cm})\end{array}$ & Unit & $\begin{array}{c}\text { Accumulation } \\
\text { Rate }(\mathrm{mm} / 1000 \mathrm{yr})\end{array}$ & Age & Name & Color & Code & Structure \\
\hline $1-1,8-10$ & 1 & & Quaternary & Marly nanno-foram ooze & Yellowish gray & $5 Y 5 / 2$ & Coarse laminae, $\mathrm{mm}$ thick \\
\hline $1-1,50-52$ & 1 & & Quaternary & Calc. sandy mud & Olive-gray & $5 Y 5 / 2$ & \\
\hline $1-3,103-105$ & 1 & & Quaternary & Calcareous mud & Olive-gray & $5 \times 3 / 2$ & Finely laminated \\
\hline $2-1,40-42$ & $2 \mathrm{~A}$ & 7 & Early Oligocene & Nanno ooze & Light greenish gray & 5 GY $8 / 1$ & Homogeneous \\
\hline $4, \mathrm{CC}$ & $2 \mathrm{~A}$ & 7 & Late Eocene & Nanno chalk & Grayish yellow green & $5 \mathrm{GY} 7 / 2$ & Homogeneous \\
\hline $5-3,49-52$ & $2 \mathrm{~B}$ & 13 & Middle Eocene & Sil. marly nanno-foram & Grayish yellow green & $5 \mathrm{GY} 7 / 2$ & Laminated \\
\hline $6-2,43-45$ & $2 \mathrm{~B}$ & 13 & Middle Eocene & Sil. nanno chalk & Light greenish gray & 5 GY $8 / 1$ & Laminated \\
\hline $6-3,15-17$ & $2 \mathrm{~B}$ & 13 & Middle Eocene & Sil. marly nanno chalk & Light greenish gray & $5 \mathrm{GY} 7 / 1$ & Bioturbated \\
\hline $7-1,113-115$ & $2 \mathrm{~B}$ & 13 & Middle Eocene & Sil. marly nanno chalk & Pale olive & $10 \mathrm{GY} 6 / 2$ & Finely laminated \\
\hline $7-2,21-23$ & $2 \mathrm{~B}$ & 13 & Middle Eocene & Sil. nanno chalk & Pale greenish yellow & $10 \mathrm{GY} 8 / 2$ & Homogeneous \\
\hline $8-3,92-94$ & $2 \mathrm{~B}$ & 13 & Middle Eocene & Sil. nanno chalk & Greenish gray & $5 \mathrm{GY} 6 / 1$ & Homogeneous \\
\hline $8-3,106-109$ & $2 \mathrm{~B}$ & 13 & Middle Eocene & Sil. nanno chalk & Greenish gray & $5 \mathrm{GY} 7 / 1$ & Zoophycos burrow (?) \\
\hline $8-4,98-100$ & $2 \mathrm{~B}$ & 13 & Middle Eocene & Sil. marly nanno chalk & Yellowish gray & 5 GY $8 / 1$ & Laminated and bioturb. \\
\hline $9 \cdot 2,137-140$ & $2 \mathrm{~B}$ & 13 & Middle Eocene & Sil. marly nanno chalk & Greenish gray & 5 GY $8 / 1$ & Burrow? pyrite cluster \\
\hline $9-2,140-143$ & $2 \mathrm{~B}$ & 13 & Middle Eocene & Sil. marly nanno chalk & Greenish gray & $5 G Y 8 / 1$ & Bioturbated \\
\hline $10-7,16-18$ & $2 \mathrm{~B}$ & 13 & Middle Eocenc & Sil. marly nanno chalk & Greenish gray & $5 \mathrm{GY} 8 / 1$ & Bioturbated \\
\hline $11-3,69-72$ & $3 \AA$ & 5 & Early Eocene & Marly foram nanno chalk & Pinkish gray & 5 YR $8 / 1$ & Foram-rich laminae \\
\hline $11-3,78-79$ & $3 \mathrm{~A}$ & 5 & Early Eocene & Marly nanno chalk & Yellowish gray & $5 \mathrm{YR} 7 / 2$ & Foram-rich laminae \\
\hline $11-4,28-31$ & $3 \mathrm{~A}$ & 5 & Larly Eocene & Marly nanno chalk & Pinkish gray & $5 \mathrm{YR} 8 / 1$ & Laminated \\
\hline $12-4,15-17$ & $3 \mathrm{~A}$ & 5 & Early Eocene & Marly foram nanno chalk & Very pale orangw & $10 \mathrm{YR} 8 / 2$ & Foram-rich laminae \\
\hline $13-1,82-85$ & $3 A$ & 5 & Early Eocenc & Nanno chalk & Grayish orange & $10 Y R 7 / 4$ & Foram-rich laminae \\
\hline $13-4,126-128$ & $3 \mathrm{~A}$ & 5 & Early Eocene & Marly nanno chalk & Very pale orange & $10 \mathrm{YR} 8 / 2$ & Laminated \\
\hline $14-2,10-12$ & $3 B$ & 5 & Late Paleocene & Marly nanno chalk & Dark yellowish brown & $10 \mathrm{YR} 4 / 2$ & Homogeneous \\
\hline $14-3,124-127$ & $3 C$ & 5 & Late Paleocene & Marly nanno chalk & Grayish orange & $10 \times \mathrm{YR} 7 / 4$ & Foram-rich laminae \\
\hline $14-5,51-53$ & $3 \mathrm{C}$ & 5 & Late Paleocene & Sil. nanno chalk & Dark yellowish brown & $10 \mathrm{YR} 4 / 2$ & Graded \\
\hline $16-2,126-129$ & $3 \mathrm{C}$ & & Middle Paleocene & Nanno chalk & Yellowish gray & $5 Y 5 / 2$ & Burrow \\
\hline $17-1,37-40$ & $3 \mathrm{C}$ & & Early Paleocene & Nanno chalk & Very pale orange & $10 \mathrm{YR} 8 / 2$ & Silty laminae at bottom \\
\hline $17-3,20-22$ & $3 \mathrm{C}$ & & Early Paleocene & Nanno chalk & Grayish orange & $10 \mathrm{YR} 7 / 4$ & Laminated (?) \\
\hline $18-1,24-26$ & $3 \mathrm{C}$ & 2 & Maestrichtian & Nanno chalk & Grayish orange & $10 \mathrm{YR} 7 / 4$ & Laminated (?) \\
\hline $19-1,118-120$ & $3 \mathrm{C}$ & 2 & Campanian & Nanno chalk & Grayish orange & $10 \mathrm{YR} 7 / 4$ & Hard ground \\
\hline
\end{tabular}

\section{Unit 1}

The recovered Quaternary sediments correspond to the upper 9.5 meters of Hole 401 (Core 1); they consist, from top to bottom, of marly calcareous ooze, calcareous ooze and mud. Two graded beds occur in the uppermost 3.5 meters. Sample 1-3, 103-105 cm, is an olive-gray (5 Y 3/2) microlaminated calcareous mud. It is rich in quartz and clay minerals. The medians of the silt fraction are, respectively, 26 and $21 \mu \mathrm{m}$.

Samples 1-1, 8-10 $\mathrm{cm}$ and $1-1,50-52 \mathrm{~cm}$ belong to a section characterized by textural sorting and color changes (Figure 5). The upper one was sampled at the top of an upward fining sequence; it is a yellowish gray ( 5 Y 7/2) laminated marly nannofossil foraminifer ooze. The lower one constitutes the base (gradational) of the graded sequence. It is an olive-gray (5 Y $5 / 2$ ) calcareous sandy mud.

The coarse fraction $(22 \%)$ of these samples comprises dominantly planktonic foraminifers. In Sample 1-1, 50-52 $\mathrm{cm}$, it accounts for 40 per cent and is characterized by the presence of rounded (13\%) and reddish $(4 \%)$ quartz (limonite coated ?) suggesting a shallow water origin for at least part of the material.

Medians of the silt fraction are, respectively, 29 and 32 $\mu \mathrm{m}$ for the bulk and 18 and $23 \mu \mathrm{m}$ for the decalcified silt, indicating active bottom current; the gradational nature of the basal contact also favors a contourite rather than a turbiditic origin for this level.

The recovered section illustrates well the changing sedimentary regime that prevailed on the margin during
Quaternary time. Detailed study of Core 1, Section 1 shows that minor cycles related to variation of the terrigenous supply occurred during periods of high carbonate production.

\section{Discussion}

Our observations suggest that the deposition of Upper Cretaceous and Paleocene sediments occurred in a regime of relatively active bottom currents and low dissolution rates. It appears that the Maestrichtian-lower Paleocene as well as the lower-upper Paleocene hiatuses resulted probably from active bottom current and/or slope instability. During the late Paleocene (Sub-unit 3B), dissolution rates increased as was also noticed at Site 400 during the same period. The increase of the sedimentation rates that occurred during the middle Eocene resulted from the conjunction of increasing terrigenous supply and a possible decrease of the dissolution rate. It thus appears that the middle/upper Eocene hiatus was not preceded by increasing bottom current intensity or dissolution rate. Late Quaternary sediments are characterized by fluctuations of the carbonate/clay ratio that probably reflected climatic and eustatic changes.

SITE 400 (Figures 2, 5, 6, 7; Tables 5A, 5B, 5C, 6, Appendix C [in back pocket])

\section{Unit 3 and Sub-Unit 2D (Cores 61 to 56, 654 to $597 \mathrm{~m}$ )}

This section is composed of reddish yellow upper Campanian to lower Maestrichtian, marly nannofossil chalk, and white to bluish white Maestrichtian chalk. 
TABLE 4B

Lithology, Site 401

\begin{tabular}{|c|c|c|c|c|c|c|c|c|c|c|c|c|c|}
\hline $\begin{array}{c}\text { Sample } \\
(\text { Interval in } \mathrm{cm})\end{array}$ & $\mathrm{CaCO}_{3}$ & Foram & Nanno & Unsp. & Quartz & $\begin{array}{l}\text { Clay, } \\
\text { Other }\end{array}$ & Silica & $\begin{array}{c}\mathrm{Md} \\
(\mu \mathrm{m})\end{array}$ & $\begin{array}{c}\text { So } \\
\text { (bulk) }\end{array}$ & $\begin{array}{c}\text { Sk } \\
\text { (bulk) }\end{array}$ & $\begin{array}{c}1 \% \\
(\mu \mathrm{m})\end{array}$ & $\begin{array}{c}\text { Md }(\mu \mathrm{m}) \\
\text { Silt } \\
\text { (bulk) }\end{array}$ & $\begin{array}{c}\text { Md }(\mu \mathrm{m}) \\
\text { Silt } \\
\text { (residual) }\end{array}$ \\
\hline $1-1,8-10$ & 63 & 49 & 13 & & 12 & 25 & & 32 & 3.96 & 0.55 & 500 & 29 & 18 \\
\hline $1-1,50-52$ & 26 & 19 & 7 & & 33 & 41 & & 50 & 3.90 & 0.95 & 400 & & \\
\hline $1-3,103-105$ & 13 & & 13 & & 25 & 62 & & 16 & 2.58 & 0.60 & 180 & 26 & 21 \\
\hline $2-1,40-42$ & 76 & 70 & & 6 & 5 & 13 & 6 & 12 & 2.58 & 0.98 & 150 & 28 & 18 \\
\hline $4, \mathrm{CC}$ & 70 & 1 & 35 & 35 & 7 & 14 & 9 & 20 & 2.58 & 0.60 & 350 & 31 & 19 \\
\hline $5-3,49-52$ & 50 & 15 & 15 & 20 & 8 & 22 & 20 & 16 & 2.31 & 0.75 & 310 & 26 & 19 \\
\hline $6-2,43-45$ & 53 & 1 & 29 & 23 & 8 & 18 & 21 & 12 & 2.28 & 0.77 & 150 & 23 & 22 \\
\hline $5-3,53-56$ & 57 & 21 & 23 & 13 & 7 & 19 & 17 & 16 & 2.58 & 0.60 & 380 & 25 & 21 \\
\hline $6-3,15-17$ & 52 & & 30 & 22 & 8 & 22 & 16 & 12 & 2.28 & 0.77 & 130 & 25 & 19 \\
\hline $7-1,113-115$ & 52 & & 22 & 30 & 12 & 22 & 12 & 16 & 2.31 & 0.75 & 130 & 29 & 20 \\
\hline $7-2,21-23$ & 55 & & 30 & 25 & 6 & 16 & 21 & 16 & 2.31 & 0.75 & 170 & 28 & 21 \\
\hline $8-3,92-94$ & 64 & & 30 & 34 & 10 & 16 & 10 & 12 & 2.58 & 0.98 & 160 & 25 & 19 \\
\hline $8-3,106-109$ & 54 & 4 & 15 & 35 & 7 & 18 & 21 & 25 & 2.24 & 0.51 & 220 & 33 & 22 \\
\hline $8-4,98-100$ & 49 & & 30 & 19 & 9 & 26 & 16 & 16 & 2.31 & 0.75 & 220 & 28 & 19 \\
\hline $9-2,137-140$ & 49 & & 20 & 29 & 8 & 24 & 17 & 20 & 2.58 & 0.60 & 160 & 29 & 19 \\
\hline $9-2,140-143$ & 49 & & 26 & 23 & 7 & 25 & 15 & 10 & 2.58 & 1.20 & 220 & 22 & 19 \\
\hline $10-7,16-18$ & 54 & 5 & 21 & 28 & 7 & 24 & 15 & 12 & 2.58 & 0.98 & 280 & 26 & 19 \\
\hline $11-3,69-72$ & 46 & 10 & 20 & 16 & 7 & 47 & & 16 & 2.18 & 0.94 & 200 & 28 & 23 \\
\hline $11-3,78-79$ & 38 & 2 & 20 & 16 & 7 & 50 & & 10 & 2.04 & 1.50 & 260 & 22 & 18 \\
\hline $11-4,28-31$ & 51 & & 30 & 21 & 7 & 42 & & 12 & 2.58 & 0.98 & 250 & 27 & 23 \\
\hline $12-4,15-17$ & 50 & 10 & 16 & 24 & 4 & 46 & & 16 & 2.58 & 0.98 & 400 & 28 & 21 \\
\hline $13-1,82-85$ & 73 & & 25 & 48 & 5 & 22 & & 20 & 2.58 & 0.60 & 400 & 30 & 15 \\
\hline $13-4,126-128$ & 55 & & 21 & 34 & 9 & 36 & & 12 & 2.9 & 0.78 & 300 & 25 & 20 \\
\hline $14-2,10-12$ & 32 & & 16 & 16 & 14 & 54 & & 16 & 2.58 & 0.60 & 180 & 27 & 19 \\
\hline $14-3,124-127$ & 31 & & 15 & 16 & 5 & 59 & & 20 & 3.98 & 0.60 & 300 & 33 & 21 \\
\hline $14-5,51-53$ & 65 & & 50 & 15 & 3 & 22 & 10 & 12 & 2.58 & 0.98 & 200 & 23 & 18 \\
\hline $16-2,126-129$ & 77 & & 52 & 25 & 1 & 15 & 7 & 16 & 2.58 & 0.94 & 180 & 28 & 21 \\
\hline $17-1,37-40$ & 93 & & 70 & 23 & & 7 & & 6 & 2.05 & 1.69 & 200 & 23 & 18 \\
\hline $17-3,20-22$ & 86 & & 46 & 40 & & 14 & & 20 & 2.89 & 0.75 & 250 & 36 & 18 \\
\hline $18-1,24-26$ & 94 & & 70 & 24 & 3 & 3 & & 6 & 1.81 & 1.31 & 250 & 22 & 17 \\
\hline $19-1,118-120$ & 73 & & 50 & 23 & & 15 & & 20 & 12.91 & 15.0 & 10000 & 17 & 19 \\
\hline
\end{tabular}

Interbedded upper Paleocene calcareous mudstone, marly nannofossil chalk, and nannofossil chalk of grayish orange color (5 YR 7/2) comprise Sub-unit 2D. They accumulated at the rate of $0.5 \mathrm{~cm} / 1000 \mathrm{yr}$.

In the coarse fraction of the Paleocene sediments (Figure 6 ), siliceous components are abundant ( 79 to $90 \%$ ). Sample $57-2,32-34 \mathrm{~cm}$ contains abundant planktonic foraminifers, showing traces of dissolution; at this level calcareous spicules, fish debris, echinoid and coral fragments, bryozoans, ostracodes, large angular or rounded quartz, and glauconite also occur. It appears that this sediment is constituted of a mixture of an in-situ pelagic component (deposited under or near the foraminifer lysocline) and shallow water reworked sediments. In contrast, the coarse fraction of the upper Maestrichtian chalk is dominantly calcareous $(59 \%$ unspecified carbonate, $30 \%$ planktonic, and $8 \%$ benthic foraminifers) and indicates a low dissolution rate. The median average of the bulk $(24 \mu \mathrm{m})$ of upper Cretaceous to Paleocene samples is badly correlated, indicating that the environmental conditions during Late Cretaceous to Paleocene evolved from low to high dissolution rates, whereas the bottom current activity was moderate to low.

\section{Sub-Unit 2C (Cores 55 to 48 )}

Lower-middle Eocene siliceous mudstone comprises most of Sub-unit 2C; it accumulated at the rate of 1.3 $\mathrm{cm} / 1000 \mathrm{yr}$. Siliceous biogenous remains reach peak concentrations up to 40 per cent in gritty layers. $\mathrm{CaCO}_{3}$ exhibits a maximum value at the base of the unit and in Core 52 so that marly chalk generally prevails in these intervals (Plate 4). This part of the section is typified by variegated greenish gray, light brown, and grayish orange sediment. Evidence of slumping and small-scale faulting occurs throughout the section; a reworked Lower Cretaceous pebble is present in Sample 48-3, 0-10 cm (Plate 4).

In the coarse fraction, siliceous components range between 16 and 99 per cent and comprise mainly radiolarians. Spicules reach up to 40 per cent at the top of the unit (Sample 47-6, 98-100 cm) which is also characterized by a high percentage $(65 \%)$ of manganese debris. Carbonate components range between 1 and 17 per cent and consist mainly of unspecified debris; benthic and planktonic foraminifers are rare (6\% and $4 \%$, respectively). The benthic foraminifers at Sample 47-6, 98-100 cm are broken and probably reworked. Quartz is rare (up to $7 \%$ ) and manganese is abundant (7\%) in Sample 47-6, 98-100 $\mathrm{cm}$. It occurs in this sample as fine debris or as coating on radiolarian tests. Muddy aggregates are also abundant and appear to be most abundant in the vicinity of hard porcellanite layers which may be related to diagenic transformation. Dissolution apparently was low during early and middle Eocene. Also noteworthy is the occurrence of spicules and manganese debris in the uppermost middle 
TABLE 4C

Lithology, Site 401

\begin{tabular}{|c|c|c|c|c|c|c|c|c|c|c|c|c|c|c|c|c|c|c|c|c|c|c|c|c|c|}
\hline $\begin{array}{c}\text { Sample } \\
\text { (Interval in } \mathrm{cm} \text { ) }\end{array}$ & QR & QRO & QA & MIC & OMIN & ROC & MUDA & PYR & GLAU & MAN & PFO & PTER & $\mathrm{BFO}$ & $\mathrm{ECH}$ & OST & LAM & BRY & COR & UNSP & RAD & DIA & SPIC & PLA & FISH & $\mathrm{OTH}$ \\
\hline \multicolumn{26}{|c|}{ Number of Particles } \\
\hline $1-1,8-10$ & 6 & 15 & 30 & 4 & 2 & 0 & 2 & 0 & 0 & 0 & 527 & 0 & 7 & 0 & 0 & 0 & 0 & 0 & 4 & 3 & 0 & 1 & 0 & 0 & 1 \\
\hline $1-1,50-52$ & 32 & 94 & 399 & 15 & 6 & 9 & 4 & 3 & 5 & 0 & 125 & 0 & 4 & 0 & 0 & 0 & 0 & 0 & 21 & 0 & 0 & 1 & 2 & 0 & 1 \\
\hline $1-3,103-105$ & 35 & 54 & 421 & 167 & 6 & 8 & 11 & 16 & 5 & 0 & 32 & 0 & 2 & 0 & 0 & 0 & 0 & 0 & 32 & 0 & 0 & 0 & 2 & 0 & 3 \\
\hline $2-1,40-42$ & 0 & $\begin{array}{r}54 \\
0\end{array}$ & $\begin{array}{r}421 \\
9\end{array}$ & 10 & 2 & ${ }_{0}^{\circ}$ & 5 & 1 & 0 & 0 & 402 & 0 & 16 & 1 & 0 & 0 & 0 & 0 & 30 & 8 & 0 & 28 & 0 & 1 & 1 \\
\hline $4, \mathrm{CC}$ & 0 & 0 & 8 & 7 & 0 & 0 & 2 & 0 & 0 & 0 & 410 & 0 & 6 & 0 & 2 & 0 & 0 & 0 & 42 & 46 & 0 & 20 & 2 & 0 & 2 \\
\hline $5-3,49-52$ & 0 & 0 & 5 & 3 & 0 & 1 & 31 & 0 & 0 & 0 & $\begin{array}{l}410 \\
429\end{array}$ & 0 & 8 & 0 & 0 & 0 & 0 & 0 & $\begin{array}{l}42 \\
29\end{array}$ & 91 & 0 & 16 & 0 & 6 & 2 \\
\hline $5 \cdot 3,53-56$ & 0 & 2 & 7 & 0 & 1 & 1 & 2 & 0 & 0 & 0 & 495 & 0 & 17 & 0 & 0 & 0 & 0 & 0 & 5 & 66 & 0 & 16 & 0 & 2 & 1 \\
\hline $6-2,43-45$ & 1 & 0 & 2 & 0 & 0 & 1 & 35 & 0 & 0 & 0 & 371 & 0 & 17 & 0 & 0 & 0 & 0 & 0 & 35 & 124 & 0 & 26 & 4 & 2 & 4 \\
\hline $6-3,15-17$ & 0 & 0 & 3 & 1 & 0 & 1 & 18 & 0 & 0 & 0 & 447 & 0 & 7 & 0 & 0 & 0 & 0 & 0 & 45 & 163 & 0 & 18 & 0 & 3 & 1 \\
\hline $7-1,113-116$ & 0 & 0 & 2 & 0 & 0 & 39 & 0 & 1 & 0 & 0 & 210 & 0 & 11 & 0 & 0 & 0 & 0 & 0 & 30 & 151 & 0 & 12 & 0 & 2 & 7 \\
\hline $7-2,21-23$ & 0 & 0 & 4 & 0 & 0 & 0 & 30 & 0 & 0 & 0 & 341 & 0 & is & 0 & 0 & 0 & 0 & 0 & 30 & 134 & 0 & 8 & 0 & 0 & 3 \\
\hline $8-3,92-94$ & 0 & 0 & 3 & 0 & 1 & 0 & 5 & 0 & 0 & 0 & 330 & 0 & 10 & 0 & 0 & 0 & 0 & 0 & 16 & 182 & 1 & 29 & 0 & 2 & 5 \\
\hline $8-3,106-109$ & 0 & 0 & 1 & 1 & 0 & 0 & 19 & 0 & 0 & 0 & 209 & 0 & 5 & 0 & 0 & 0 & 0 & 0 & 29 & 233 & 0 & 16 & 3 & 4 & 1 \\
\hline $8-4,98-100$ & 0 & 0 & $i$ & 0 & 0 & 0 & 25 & 0 & 0 & 0 & 241 & 0 & 11 & 0 & 0 & 0 & 0 & 0 & 22 & 158 & 0 & 18 & 0 & 2 & 2 \\
\hline $9-2,137-140$ & 0 & 0 & $i$ & 0 & 0 & 0 & 4 & 29 & 0 & 0 & 229 & 0 & 4 & 1 & 1 & 0 & 0 & 0 & 17 & 271 & 0 & 20 & 0 & $i$ & 11 \\
\hline $9-2,140-143$ & 0 & 0 & $i$ & 0 & 0 & 0 & 6 & 0 & 0 & 0 & 283 & 0 & 13 & 0 & 0 & 0 & 0 & 0 & 19 & 148 & 2 & 10 & 0 & 2 & 8 \\
\hline $10-7,16-18$ & 0 & 0 & 0 & 0 & 0 & 0 & 4 & 1 & 0 & 0 & 348 & 0 & 11 & 0 & 0 & 0 & 0 & 0 & 13 & 138 & 0 & 22 & 0 & 6 & 17 \\
\hline $11-3,69-72$ & 0 & 1 & 0 & 0 & 0 & 0 & 10 & 0 & 0 & 0 & 517 & 0 & 10 & 0 & 0 & 0 & 0 & 0 & 11 & 0 & 0 & 0 & 0 & 2 & 3 \\
\hline $11-3,78-79$ & 0 & 0 & 0 & 0 & 0 & 0 & 29 & 0 & 0 & 0 & 389 & 0 & 10 & 0 & 0 & 0 & 0 & 0 & $\begin{array}{l}11 \\
16\end{array}$ & 0 & 0 & 1 & 0 & 0 & 1 \\
\hline $\mid 1-4,28-31$ & 0 & 0 & $i$ & 0 & 0 & 0 & 8 & 0 & 0 & 0 & 301 & 0 & 4 & 0 & 0 & 0 & 0 & 0 & 39 & 169 & 0 & 2 & 0 & 1 & 7 \\
\hline $12-4,15-17$ & 0 & 0 & i & 0 & 1 & 0 & 3 & 0 & 0 & 0 & 464 & 0 & 8 & 0 & 0 & 0 & 0 & 0 & 16 & 10 & 0 & 0 & 0 & 0 & 0 \\
\hline $13-1,82-85$ & 0 & 0 & 1 & 1 & 0 & 0 & 2 & 0 & 0 & 1 & 614 & 0 & 2 & 0 & 0 & 0 & 0 & 0 & 12 & 3 & 0 & 0 & 0 & 0 & 1 \\
\hline $13-4,126-128$ & 0 & 0 & 6 & 1 & 0 & 3 & 23 & 3 & 0 & 0 & 609 & 0 & 37 & 0 & 1 & 0 & 0 & 0 & 15 & 1 & 0 & 0 & 0 & 0 & 4 \\
\hline $14-2,10-12$ & 1 & 0 & 10 & 2 & 0 & 0 & 7 & 0 & 0 & 0 & 482 & 0 & 7 & 0 & i & 0 & 0 & 0 & 4 & 0 & 0 & 1 & 0 & 3 & i \\
\hline $14-3,124-127$ & 0 & 0 & 2 & $i$ & 0 & 0 & 1 & 0 & 0 & 0 & 519 & 0 & 5 & 0 & 0 & 0 & 0 & 0 & 21 & 0 & 0 & 0 & 0 & 1 & 2 \\
\hline $14-5,51-53$ & 0 & 0 & 2 & 9 & 0 & 1 & 27 & 0 & 0 & 0 & 505 & 0 & 5 & 0 & 1 & 0 & 0 & 0 & 26 & 13 & 0 & 49 & 0 & 2 & 8 \\
\hline $16-2,126-129$ & 0 & 0 & 0 & 1 & 0 & 0 & 44 & 0 & 0 & 0 & 340 & 0 & 3 & 0 & 0 & 0 & 0 & 0 & 23 & 98 & 0 & 29 & 0 & 0 & 4 \\
\hline $16-2,126-129$ & 0 & 0 & 1 & 1 & 0 & 0 & 43 & 0 & 0 & 0 & 453 & 0 & 4 & i & 2 & 0 & 0 & 0 & 27 & 50 & 0 & 22 & 0 & 0 & 2 \\
\hline $17-1,37-40$ & 0 & 0 & 0 & 0 & 0 & 0 & 15 & 0 & 0 & 2 & 433 & 0 & 4 & 0 & 0 & 1 & 0 & 0 & 26 & 0 & 0 & 1 & 0 & 0 & 0 \\
\hline $17-3,20-22$ & 0 & 0 & 0 & 1 & 0 & 0 & 1 & 0 & 0 & 0 & 568 & 0 & 6 & 0 & 2 & 0 & 0 & 0 & 8 & 0 & 0 & 0 & 0 & 0 & 1 \\
\hline $18-1,24-26$ & 0 & 0 & 0 & 1 & 0 & 0 & 13 & 0 & 0 & 1 & 420 & 0 & 12 & 0 & 0 & 0 & 0 & 0 & $\begin{array}{l}\circ \\
49\end{array}$ & 1 & 0 & 1 & 0 & 0 & 1 \\
\hline $19-1,118-120$ & 1 & 0 & 1 & 4 & 128 & 40 & 17 & 0 & 0 & 51 & 16 & 0 & 8 & 10 & 0 & 0 & 0 & 1 & 69 & 0 & 0 & 0 & 0 & 0 & 0 \\
\hline \multicolumn{26}{|c|}{ Particles Frequencies } \\
\hline $1-1,8-10$ & 1.0 & 2.5 & 5.0 & 0.7 & 0.3 & 0.0 & 0.3 & 0.0 & 0.0 & 0.0 & 87.5 & 0.0 & 1.2 & 0.0 & 0.0 & 0.0 & 0.0 & 0.0 & 0.7 & 0.5 & 0.0 & 0.2 & 0.0 & 0.0 & 0.2 \\
\hline $1-1,50-52$ & 4.4 & 13.0 & 55.3 & 2.1 & 0.8 & 1.2 & 0.6 & 0.4 & 0.7 & 0.0 & 17.3 & 0.0 & 0.6 & 0.0 & 0.0 & 0.0 & 0.0 & 0.0 & 2.9 & 0.0 & 0.0 & 0.1 & 0.3 & 0.0 & 0.1 \\
\hline $1-3,103-105$ & 4.4 & 6.8 & 53.0 & 21.0 & 0.8 & 1.0 & 1.4 & 2.0 & 0.6 & 0.0 & 4.0 & 0.0 & 0.3 & 0.0 & 0.0 & 0.0 & 0.0 & 0.0 & 4.0 & 0.0 & 0.0 & 0.0 & 0.3 & 0.0 & 0.4 \\
\hline $2-1,40-42$ & 0.0 & 0.0 & 1.8 & 1.9 & 0.4 & 0.0 & 1.0 & 0.2 & 0.0 & 0.0 & 78.2 & 0.0 & 3.1 & 0.2 & 0.0 & 0.0 & 0.0 & 0.0 & 5.8 & 1.6 & 0.0 & 5.4 & 0.0 & 0.2 & 0.2 \\
\hline $4, \mathrm{CC}$ & 0.0 & 0.0 & 1.5 & 1.3 & 0.0 & 0.0 & 0.4 & 0.0 & 0.0 & 0.0 & 75.0 & 0.0 & 1.1 & 0.0 & 0.4 & 0.0 & 0.0 & 0.0 & 7.7 & 8.4 & 0.0 & 3.7 & 0.4 & 0.0 & 0.4 \\
\hline $5 \cdot 3,49-52$ & 0.0 & 0.0 & 0.8 & 0.5 & 0.0 & 0.2 & 5.0 & 0.0 & 0.0 & 0.0 & 69.1 & 0.0 & 1.3 & 0.0 & 0.0 & 0.0 & 0.0 & 0.0 & 4.7 & $\begin{array}{r}0.4 \\
14.7\end{array}$ & 0.0 & 2.6 & 0.0 & 1.0 & 0.3 \\
\hline $5 \cdot 3,53-56$ & 0.0 & 0.3 & $\begin{array}{l}1.0 \\
1.1\end{array}$ & 0.0 & 0.2 & 0.2 & 0.3 & 0.0 & 0 & 0.0 & 80.5 & 0.0 & 2.8 & 0.0 & 0.0 & $\begin{array}{l}0.0 \\
0.0\end{array}$ & 0.0 & 0.0 & 0.8 & 10.7 & 0.0 & 2.6 & .0 & $\begin{array}{l}1.0 \\
0.3\end{array}$ & 0.2 \\
\hline $6-2,43-45$ & 0.2 & 0.0 & 0.3 & 0.0 & 0.0 & $\begin{array}{l}0.2 \\
0.2\end{array}$ & 5.6 & 0.0 & 0. & 0.0 & 59.6 & 0.0 & 2.7 & .0 & 0.0 & 0.0 & 0.0 & 0.0 & $\begin{array}{l}.08 \\
5.6\end{array}$ & 19.9 & .0 & $\begin{array}{l}2.0 \\
4.2\end{array}$ & 6 & $\begin{array}{l}0.3 \\
0.3\end{array}$ & 0.6 \\
\hline $6-3,15-17$ & 0.0 & 0.0 & 0. & 0. & 0. & 0.1 & 2.5 & 0.0 & 0. & 0.0 & 63.2 & 0.0 & 1.0 & 0.0 & 0.0 & 0. & 0.0 & 0 & 6.4 & 23.1 & 0.0 & 2.5 & 0 & 0.4 & 0.1 \\
\hline $7-1,113-116$ & 0.0 & 0.0 & 0.4 & 0.0 & 0. & 8.4 & 0.0 & 0. & 0.6 & 0 . & 45.2 & 0. & 2.4 & 0.0 & 0.0 & 0. & 0. & 0. & 6.5 & 32.5 & 0.0 & 2.6 & 0.0 & 0.4 & 1.5 \\
\hline $7-2,21-2$ & 0. & 0.0 & 0.7 & 0.0 & 0 . & 0. & 5.3 & 0.0 & 0. & 0.0 & 60.4 & 0. & 2.7 & 0.0 & 0. & 0. & 0 & 0 . & 5.3 & 23.7 & 0.0 & 1.4 & 0.0 & 0.0 & 0.5 \\
\hline $8-3,92-5$ & 0. & 0. & 0.5 & 0.0 & 0. & 0. & 0.9 & 0.0 & 0. & 0.0 & 56.5 & 0. & 1.7 & 0.0 & 0. & 0. & 0. & 0. & 2.7 & 31.2 & 0.2 & 5.0 & 0.0 & 0.3 & 0.9 \\
\hline $8-3,106-109$ & 0. & 0. & 0.2 & 0.2 & 0. & 0. & 3.6 & 0.0 & 0. & 0.0 & 40.1 & 0. & 1.0 & 0.0 & 0. & 0 . & 0. & 0. & 5.6 & 44.7 & 0.0 & 3.1 & 0.6 & 0.8 & 0.2 \\
\hline $8-4,98$ & 0.0 & 0. & 0.4 & 0.0 & 0.0 & 0.0 & 5.2 & 0.0 & 0. & 0.0 & 50.1 & 0. & 2.3 & 0.0 & 0. & 0. & 0. & 0. & 4.6 & 32.8 & 0.0 & 3.7 & 0.0 & 0.4 & 0.4 \\
\hline $9-2,137-140$ & 0.0 & 0.0 & 0.2 & 0.0 & 0.0 & 0.0 & 0.7 & 4.9 & 0.0 & 0.0 & 38.9 & 0.0 & 0.7 & 0.2 & 0.2 & 0.0 & 0.0 & 0.0 & 2.9 & 46.0 & 0.0 & 3.4 & 0.0 & 0.2 & 1.9 \\
\hline $9-2,140-143$ & 0.0 & 0.0 & 0.2 & 0.0 & 0.0 & 0.0 & 1.2 & 0.0 & 0.0 & 0.0 & 57.5 & 0.0 & 2.6 & 0.0 & 0.0 & 0.0 & 0.0 & 0.0 & 3.9 & 30.1 & 0.4 & 2.0 & 0.0 & 0.4 & 1.6 \\
\hline $10-7,16-18$ & 0.0 & 0.0 & 0.0 & 0.0 & 0.0 & 0.0 & 0.7 & 0.2 & 0.0 & 0.0 & 62.1 & 0.0 & 2.0 & 0.0 & 0.0 & 0.0 & 0.0 & 0.0 & 2.3 & 24.6 & 0.0 & 3.9 & 0.0 & 1.1 & 3.0 \\
\hline $11-3,69-72$ & 0.0 & 0.2 & 0.0 & 0.0 & 0.0 & 0.0 & 1.8 & 0.0 & 0.0 & 0.0 & 93.3 & 0.0 & 1.8 & 0.0 & 0.0 & 0.0 & 0.0 & 0.0 & 2.0 & 0.0 & 0.0 & 0.0 & 0.0 & 0.4 & 0.5 \\
\hline
\end{tabular}




\begin{tabular}{|c|c|c|c|c|c|c|c|c|c|c|c|c|c|c|c|c|c|c|c|c|c|c|c|c|c|}
\hline $11-3,78-79$ & 0.0 & 0.0 & 0.0 & 0.0 & 0.0 & 0.0 & 6.5 & 0.0 & 0.0 & 0.0 & 87.2 & 0.0 & 2.2 & 0.0 & 0.0 & 0.0 & 0.0 & 0.0 & 3.6 & 0.0 & 0.0 & 0.2 & 0.0 & 0.0 & 0.2 \\
\hline $11-4,28-31$ & 0.0 & 0.0 & 0.2 & 0.0 & 0.0 & 0.0 & 1.5 & 0.0 & 0.0 & 0.0 & 56.6 & 0.0 & 0.8 & 0.0 & 0.0 & 0.0 & 0.0 & 0.0 & 7.3 & 31.8 & 0.0 & 0.4 & 0.0 & 0.2 & 1.3 \\
\hline $12-4,15-17$ & 0.0 & 0.0 & 0.2 & 0.0 & 0.2 & 0.0 & 0.6 & 0.0 & 0.0 & 0.0 & 92.2 & 0.0 & 1.6 & 0.0 & 0.0 & 0.0 & 0.0 & 0.0 & 3.2 & 2.0 & 0.0 & 0.0 & 0.0 & 0.0 & 0.0 \\
\hline $13-1,82-85$ & 0.0 & 0.0 & 0.2 & 0.2 & 0.0 & 0.0 & 0.3 & 0.0 & 0.0 & 0.2 & 96.4 & 0.0 & 0.3 & 0.0 & 0.0 & 0.0 & 0.0 & 0.0 & 1.9 & 0.5 & 0.0 & 0.0 & 0.0 & 0.0 & 0.2 \\
\hline $13-4,126-128$ & 0.0 & 0.0 & 0.9 & 0.1 & 0.0 & 0.4 & 3.3 & 0.4 & 0.0 & 0.0 & 86.6 & 0.0 & 5.3 & 0.0 & 0.1 & 0.0 & 0.0 & 0.0 & 2.1 & 0.1 & 0.0 & 0.0 & 0.0 & 0.0 & 0.6 \\
\hline $14-2,10-12$ & 0.2 & 0.0 & 1.9 & 0.4 & 0.0 & 0.0 & 1.3 & 0.0 & 0.0 & 0.0 & 92.9 & 0.0 & 1.3 & 0.0 & 0.2 & 0.0 & 0.0 & 0.0 & 0.8 & 0.0 & 0.0 & 0.2 & 0.0 & 0.6 & 0.2 \\
\hline $14-3,124-127$ & 0.0 & 0.0 & 0.4 & 0.2 & 0.0 & 0.0 & 0.2 & 0.0 & 0.0 & 0.0 & 94.0 & 0.0 & 0.9 & 0.0 & 0.0 & 0.0 & 0.0 & 0.0 & 3.8 & 0.0 & 0.0 & 0.0 & 0.0 & 0.2 & 0.4 \\
\hline $14-5,51-53$ & 0.0 & 0.0 & 0.3 & 1.4 & 0.0 & 0.2 & 4.2 & 0.0 & 0.0 & 0.0 & 77.9 & 0.0 & 0.8 & 0.0 & 0.2 & 0.0 & 0.0 & 0.0 & 4.0 & 2.0 & 0.0 & 7.6 & 0.0 & 0.3 & 1.2 \\
\hline $16-2,126-129$ & 0.0 & 0.0 & 0.0 & 0.2 & 0.0 & 0.0 & 8.1 & 0.0 & 0.0 & 0.0 & 62.7 & 0.0 & 0.6 & 0.0 & 0.0 & 0.0 & 0.0 & 0.0 & 4.2 & 18.1 & 0.0 & 5.4 & 0.0 & 0.0 & 0.7 \\
\hline $16-2,126-129$ & 0.0 & 0.0 & 0.2 & 0.2 & 0.0 & 0.0 & 7.1 & 0.0 & 0.0 & 0.0 & 74.8 & 0.0 & 0.7 & 0.2 & 0.3 & 0.0 & 0.0 & 0.0 & 4.5 & 8.3 & 0.0 & 3.6 & 0.0 & 0.0 & 0.3 \\
\hline $17 \cdot 1,37-40$ & 0.0 & 0.0 & 0.0 & 0.0 & 0.0 & 0.0 & 3.1 & 0.0 & 0.0 & 0.4 & 89.8 & 0.0 & 0.8 & 0.0 & 0.0 & 0.2 & 0.0 & 0.0 & 5.4 & 0.0 & 0.0 & 0.2 & 0.0 & 0.0 & 0.0 \\
\hline $17-3,20-22$ & 0.0 & 0.0 & 0.0 & 0.2 & 0.0 & 0.0 & 0.2 & 0.0 & 0.0 & 0.0 & 96.8 & 0.0 & 1.0 & 0.0 & 0.3 & 0.0 & 0.0 & 0.0 & 1.4 & 0.0 & 0.0 & 0.0 & 0.0 & 0.0 & 0.0 \\
\hline $18-1,24-26$ & 0.0 & 0.0 & 0.0 & 0.2 & 0.0 & 0.0 & 2.6 & 0.0 & 0.0 & 0.2 & 84.2 & 0.0 & 2.4 & 0.0 & 0.0 & 0.0 & 0.0 & 0.0 & 9.8 & 0.2 & 0.0 & 0.2 & 0.0 & 0.0 & 0.2 \\
\hline $19-1,118-120$ & 0.3 & 0.0 & 0.3 & 1.2 & 37.0 & 11.6 & 4.9 & 0.0 & 0.0 & 14.7 & 4.6 & 0.0 & 2.3 & 2.9 & 0.0 & 0.0 & 0.0 & 0.3 & 19.9 & 0.0 & 0.0 & 0.0 & 0.0 & 0.0 & 0.0 \\
\hline
\end{tabular}

\begin{tabular}{|c|c|c|c|c|c|}
\hline & $\begin{array}{l}\text { Terrigenous } \\
(\%)\end{array}$ & $\begin{array}{l}\text { Authigenic } \\
(\%)\end{array}$ & $\begin{array}{c}\text { Calcareous } \\
(\%)\end{array}$ & $\begin{array}{c}\text { Siliceous } \\
(\%)\end{array}$ & $\begin{array}{c}\text { Miscellaneous } \\
(\%)\end{array}$ \\
\hline $1-1,8-10$ & 9.80 & 0.00 & 89.37 & 0.66 & 0.17 \\
\hline $1-1,50-52$ & 77.53 & 1.11 & 20.80 & 0.14 & 0.42 \\
\hline $1.3,103-105$ & 88.41 & 264 & 8.31 & 0.00 & 0.63 \\
\hline $2-1,40-42$ & 5.06 & 0.19 & 87.35 & 7.00 & 0.39 \\
\hline 4. CC & 3.11 & 0.00 & 84.10 & 12.07 & 0.73 \\
\hline $5-3,49-52$ & $\begin{array}{l}6.44 \\
6.44\end{array}$ & 0.00 & 75.04 & 17.23 & 1.29 \\
\hline $5-3,53-56$ & 2.11 & 0.00 & 84.07 & 13.33 & 0.49 \\
\hline $6-2,43-45$ & 6.27 & 0.00 & $\begin{array}{l}68.01 \\
68.01\end{array}$ & 24.12 & 1.61 \\
\hline $6-3,15-17$ & 3.25 & 0.00 & 70.58 & 25.60 & $\begin{array}{l}1.01 \\
0.57\end{array}$ \\
\hline $7-1,113-116$ & 8.82 & 0.22 & 53.98 & 35.05 & 1.94 \\
\hline $7-2,21-23$ & 6.02 & 0.00 & 68.32 & 25.13 & 0.53 \\
\hline $8-3,92-94$ & 1.54 & 0.00 & 60.96 & 36.30 & 1.20 \\
\hline $8-3,106-109$ & 4.03 & 0.00 & 46.64 & 47.79 & 1.54 \\
\hline $8-4,98-100$ & 5.61 & 0.00 & 56.96 & 36.59 & 0.83 \\
\hline $9-2,137-140$ & 0.85 & 4.92 & 42.78 & 49.41 & 2.04 \\
\hline $9-2,140-143$ & 1.42 & 0.00 & 64.02 & 32.52 & 2.03 \\
\hline $10-7,16-18$ & 0.71 & 0.18 & 66.43 & 28.57 & 4.11 \\
\hline $11-3,69-72$ & 1.99 & $\begin{array}{l}0.18 \\
0.00\end{array}$ & $\begin{array}{l}60.43 \\
97.11\end{array}$ & $\begin{array}{r}28.51 \\
0.00\end{array}$ & $\begin{array}{l}4.11 \\
0.90\end{array}$ \\
\hline $11-3,78-79$ & 6.50 & 0.00 & 93.05 & 0.22 & 0.22 \\
\hline $11-4,28-31$ & $\begin{array}{l}1.69 \\
1.6\end{array}$ & 0.00 & 64.66 & 32.14 & 1.50 \\
\hline $12-4,15-17$ & 0.99 & 0.00 & 97.02 & $\begin{array}{r}1.99 \\
1.99\end{array}$ & 0.00 \\
\hline $13-1,82-85$ & 0.63 & 0.16 & 98.59 & 0.47 & 0.16 \\
\hline $13-4,126-128$ & $\begin{array}{l}8 \quad 4.69 \\
\end{array}$ & 0.43 & 94.17 & 0.14 & 0.57 \\
\hline $14-2,10-12$ & 3.85 & 0.00 & 95.18 & 0.19 & 0.77 \\
\hline $14-3,124-127$ & 0.72 & 0.00 & 98.73 & 0.00 & 0.54 \\
\hline $14-5,51-53$ & 6.02 & 0.00 & 82.87 & 9.57 & 1.54 \\
\hline $16-2,126-129$ & 8.30 & 0.00 & 67.53 & 23.43 & 0.74 \\
\hline $16-2,126-129$ & $\begin{array}{l}8.30 \\
7.43\end{array}$ & $\begin{array}{l}0.00 \\
0.00\end{array}$ & 80.36 & $\begin{array}{l}23.43 \\
11.88\end{array}$ & $\begin{array}{l}0.14 \\
0.33\end{array}$ \\
\hline $17-1,37-40$ & 3.11 & 0.41 & 96.27 & 0.21 & 0.00 \\
\hline $17-3,20-22$ & 0.34 & 0.00 & 99.49 & 0.00 & 0.17 \\
\hline $18-1,24-26$ & 2.81 & 0.20 & 96.39 & 0.40 & 0.20 \\
\hline $19-1,118-120$ & $\begin{array}{r}2.61 \\
55.20\end{array}$ & $\begin{array}{l}0.20 \\
14.74\end{array}$ & 30.06 & $\begin{array}{l}0.40 \\
0.00\end{array}$ & $\begin{array}{l}0.20 \\
0.00\end{array}$ \\
\hline
\end{tabular}

Note: $Q R=$ reddish quartz, $Q R O=$ rounded quartz, $Q A=$ angulous quartz, $M I C=$ mica, $\mathrm{OMIN}=$ other minerals, $\mathrm{ROC}=$ rock fragments, $\mathrm{MUDA}=$ muddy aggregates; Authigenic: $\mathrm{PYR}=$ pyrite, $\mathrm{GLAU}=$ glauconite, $\mathrm{MAN}=$ manganese; Calcar -

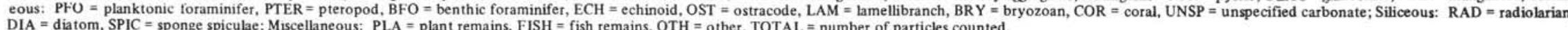




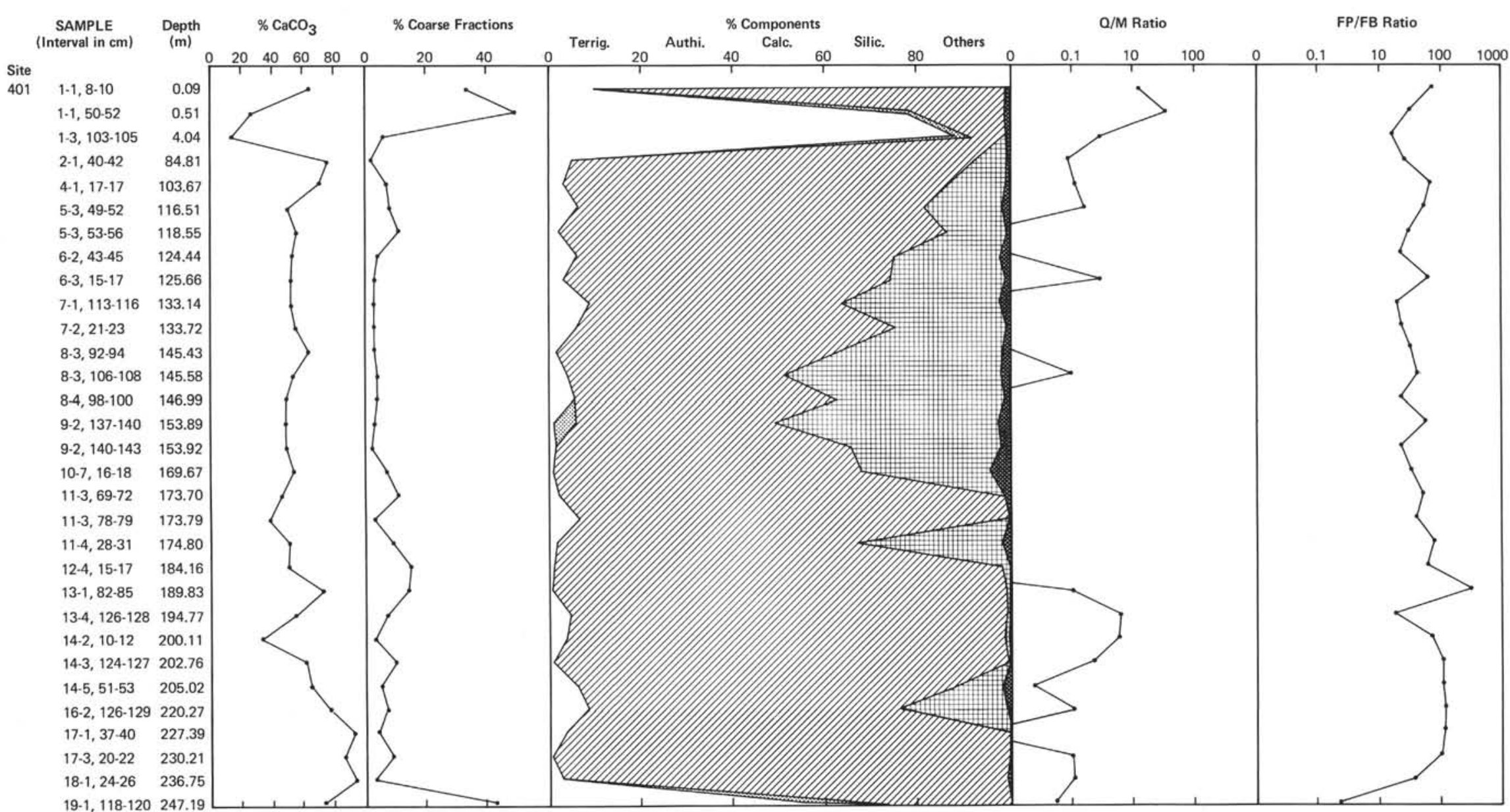

Figure 4. Coarse fraction, Site 401. 
LITHOLOGY

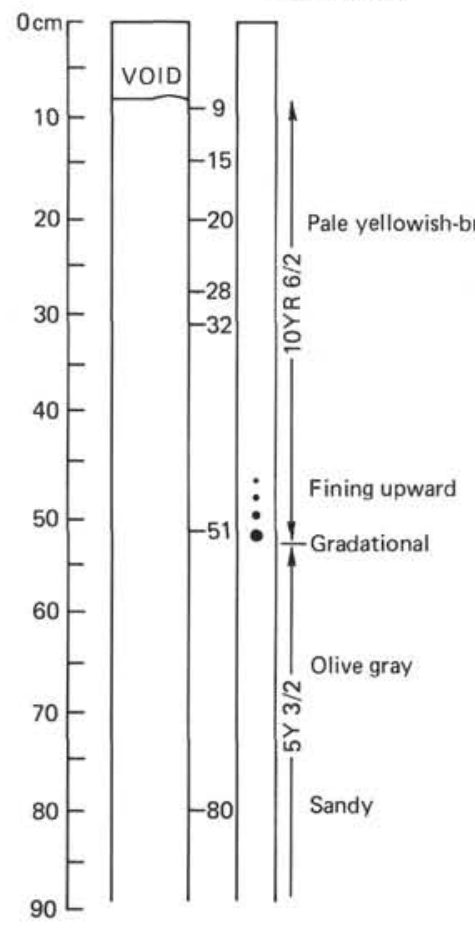

TEXTURE

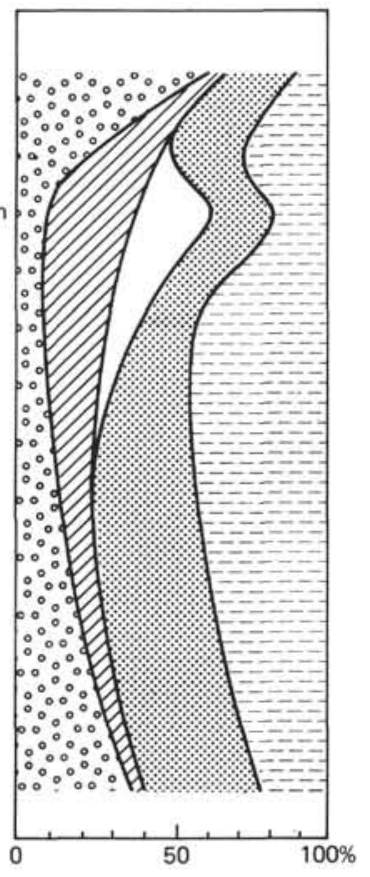

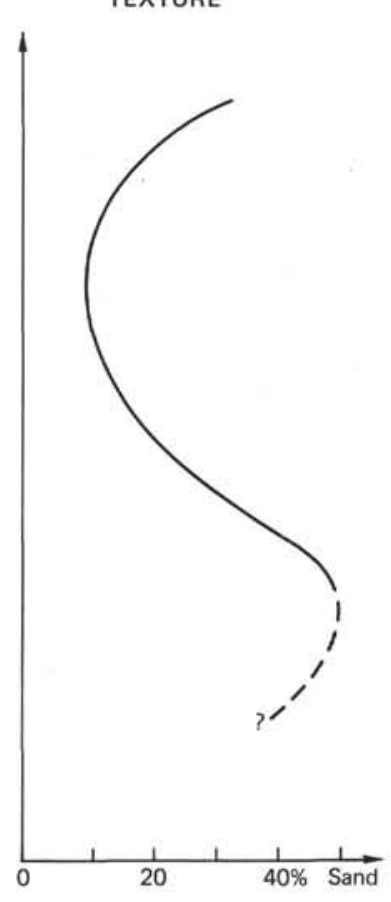

Figure 5. Lithology Hole 401, Core 1, Section 1.

Eocene (Sample 49-4, 64-66 cm). Median averages of the bulk and decalcified silt fraction of the lower Eocene sediment are in the same range $(23 \mu \mathrm{m})$; the correlation coefficient increases notably $(0.51)$, thus suggesting enhancement of the hydrodynamical control of the particle size of the sediment. Textural parameters of the middle Eocene sediments are quite similar to those of the lower Eocene. The correlation coefficient increases slightly $(0.6)$ thus pointing to a possible strengthening of the bottom current, further indicated by concentration of sponge spicules in gritty layers. This enhancement of the bottom current intensity preceded the late Eocene hiatus which may thus be explained (at least in part) by sediment erosion.

\section{Sub-Unit 2B (Cores 47 to 42 )}

This sub-unit comprises lower to upper Oligocene siliceous marly nannofossil chalk. The base of the sub-unit is characterized by an abrupt color change from dominantly grayish orange to yellowish gray. The sediment accumulated at the low rate of $0.35 \mathrm{~cm} / 1000 \mathrm{yr}$. Silty layers containing up to 30 per cent foraminifers and 35 per cent sponge spicules occur throughout; the layers range up to 3 $\mathrm{cm}$ in thickness and are often laminated, cross-laminated, and graded (Plate 4). Their base is often sharp, but their upper boundary is gradational. In Core 43 , where coarse layers are common and spaced at intervals of 10 to $20 \mathrm{~cm}$, evidence of probable syndepositional slump occurs (Plate $4)$.
In the coarse fraction, the terrigenous component is generally low (4 to $23 \%$ ) except in the lowermost Oligocene marly chalk where it reaches 46 to 68 per cent including mostly angular and few $(2 \%)$ reddish subrounded quartz. The carbonate component ranges between 18 and 85 per cent; it includes benthic foraminifers ( 8 to $60 \%$ ) unspecified debris ( 7 to $48 \%$ ) (unspecified debris results probably from the fragmentation of foraminifers) and planktonic foraminifers; noteworthy is the absence of agglutinated foraminifers. The dissolution level though difficult to evaluate, must have been at least moderate. The siliceous component ranges between 3 and 68 per cent and averages about 35 per cent; it comprises 16 to 69 per cent sponge spicules and 3 per cent radiolarians. Frequencies of quartz and spicules are positively correlated, but both are negatively correlated to the benthic foraminifer frequencies. It appears that periods of high terrigenous supply and strong bottom current, favoring sponge development, may have alternated with periods of current weakening and lesser terrigenous supply more propitious to the existence of benthic foraminifers.

The average of the silt medians of both bulk and residual fraction increases notably ( 36 and $26 \mu \mathrm{m}$, respectively) but the correlation between them is poor $(-0.09)$. As previously deduced from the coarse fraction observations, this may be consequent upon fluctuation of the hydrodynamic (and hydrologic) conditions. It thus appears that the sediment of Sub-unit 2B was deposited under active, though fluctuating, bottom current conditions, and 
TABLE 5A

Lithology, Holes 400/400A

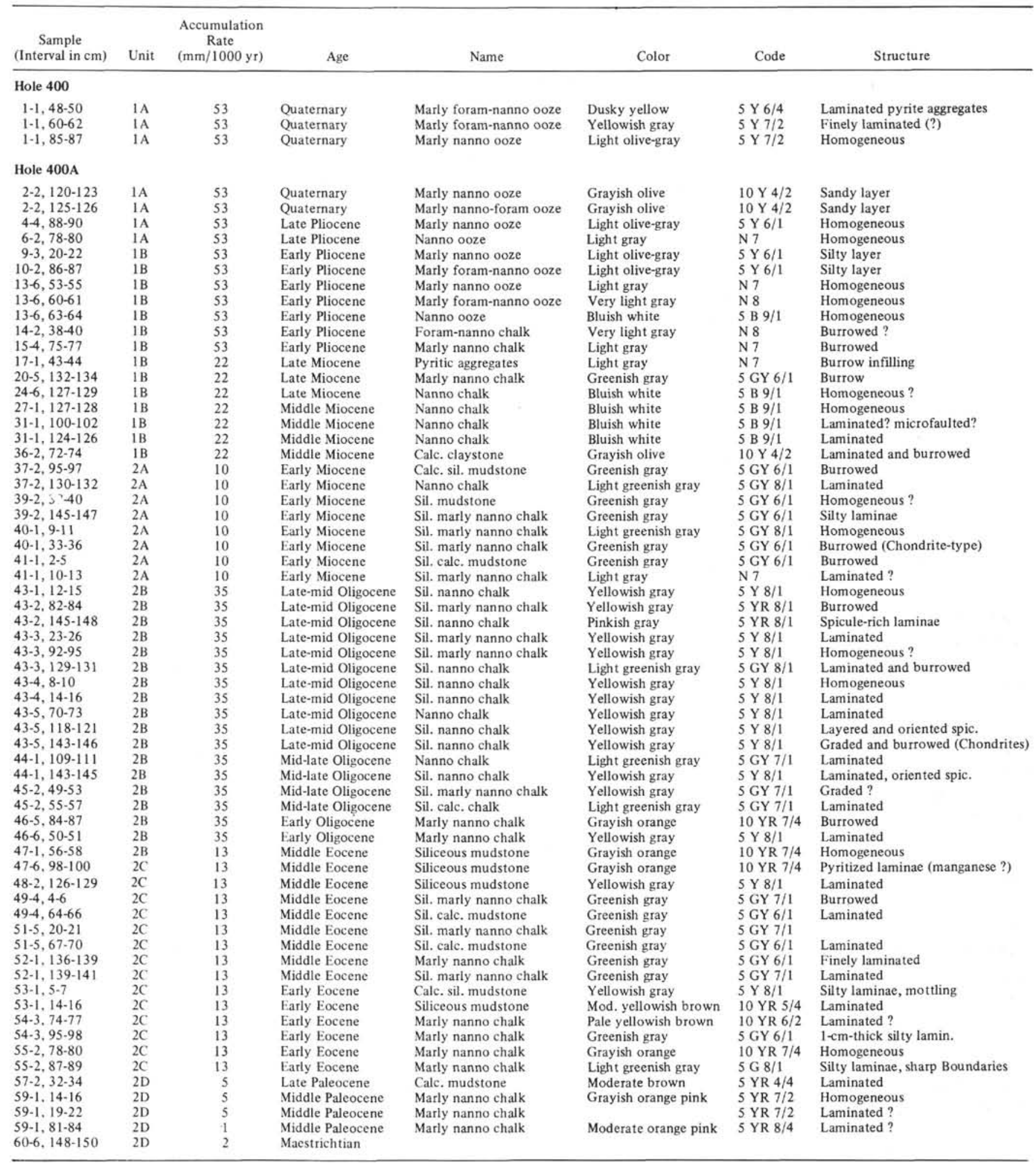


TABLE 5B

Lithology, Holes 400/400A

\begin{tabular}{|c|c|c|c|c|c|c|c|c|c|c|c|c|c|}
\hline $\begin{array}{c}\text { Sample } \\
\text { (Interval in cm) }\end{array}$ & $\mathrm{CaCO}_{3}$ & Foraminifer & Nannofossil & Unsp. & Quartz & $\begin{array}{l}\text { Clay, } \\
\text { Other }\end{array}$ & Silica & $\begin{array}{l}\text { Md } \\
(\mu \mathrm{m})\end{array}$ & $\begin{array}{c}\text { So } \\
\text { (bulk) }\end{array}$ & $\begin{array}{c}\text { Sk } \\
\text { (bulk) }\end{array}$ & $\begin{array}{l}1 \% \\
(\mu \mathrm{m})\end{array}$ & $\begin{array}{l}\text { Md }(\mu \mathrm{m}) \\
\text { Silt } \\
\text { (bulk) }\end{array}$ & $\begin{array}{l}\text { Md }(\mu \mathrm{m}) \\
\text { Silt } \\
\text { (residual) }\end{array}$ \\
\hline \multicolumn{14}{|l|}{ Hole 400} \\
\hline $\begin{array}{l}1-1,48-50 \\
1-1,60-62 \\
1-1,85-87\end{array}$ & $\begin{array}{l}56 \\
54 \\
38\end{array}$ & $\begin{array}{r}10 \\
35 \\
7\end{array}$ & $\begin{array}{l}30 \\
15 \\
25\end{array}$ & $\begin{array}{r}16 \\
4 \\
6\end{array}$ & $\begin{array}{r}16 \\
8 \\
5\end{array}$ & $\begin{array}{l}21 \\
37 \\
55\end{array}$ & & $\begin{array}{l}13 \\
16 \\
10\end{array}$ & $\begin{array}{l}2.04 \\
2.04 \\
2.29\end{array}$ & $\begin{array}{l}0.61 \\
0.59 \\
0.76\end{array}$ & $\begin{array}{l}200 \\
200 \\
200\end{array}$ & $\begin{array}{l}20 \\
23 \\
18\end{array}$ & $\begin{array}{l}33 \\
18\end{array}$ \\
\hline \multicolumn{14}{|l|}{ Hole 400A } \\
\hline $\begin{array}{c}2-2,120-123 \\
2-2,125-126 \\
4-4,88-90 \\
6-2,78-80 \\
9-3,20-22 \\
10-2,86-87 \\
13-6,53-55 \\
13-6,60-61 \\
13-6,63-64 \\
14-2,38-40 \\
15-4,75-77 \\
17-1,43-44\end{array}$ & $\begin{array}{l}31 \\
41 \\
43 \\
73 \\
49 \\
44 \\
54 \\
61 \\
78 \\
72 \\
60\end{array}$ & $\begin{array}{r}27 \\
30 \\
2 \\
5 \\
10 \\
10 \\
5 \\
10 \\
3 \\
20\end{array}$ & $\begin{array}{r}3 \\
10 \\
30 \\
50 \\
22 \\
10 \\
35 \\
30 \\
50 \\
40 \\
45\end{array}$ & $\begin{array}{r}1 \\
1 \\
11 \\
18 \\
17 \\
24 \\
14 \\
21 \\
25 \\
12 \\
15\end{array}$ & $\begin{array}{r}16 \\
20 \\
29 \\
7 \\
10 \\
11 \\
7 \\
5 \\
3 \\
4 \\
6\end{array}$ & $\begin{array}{l}53 \\
39 \\
25 \\
20 \\
36 \\
30 \\
39 \\
34 \\
19 \\
18 \\
34\end{array}$ & $\begin{array}{l}4 \\
5\end{array}$ & $\begin{array}{r}40 \\
25 \\
6 \\
13 \\
13 \\
25 \\
13 \\
16 \\
16 \\
13 \\
8\end{array}$ & $\begin{array}{l}2.42 \\
2.43 \\
2.31 \\
2.56 \\
2.9 \\
1.58 \\
2.28 \\
1.79 \\
2.0 \\
2.88 \\
2.29\end{array}$ & $\begin{array}{l}0.94 \\
1.20 \\
1.33 \\
0.61 \\
0.78 \\
1.02 \\
0.77 \\
1.25 \\
1.0 \\
0.77 \\
1.19\end{array}$ & $\begin{array}{r}220 \\
450 \\
56 \\
270 \\
140 \\
1000 \\
70 \\
70 \\
76 \\
200 \\
50\end{array}$ & $\begin{array}{l}25 \\
24 \\
21 \\
22 \\
24 \\
27 \\
25 \\
21 \\
23 \\
23 \\
23\end{array}$ & $\begin{array}{l}25 \\
20 \\
19 \\
18 \\
28 \\
26 \\
22 \\
19 \\
17 \\
20 \\
18\end{array}$ \\
\hline $20-5,132-134$ & 50 & & 40 & 10 & 7 & 41 & 2 & 5 & 2.31 & 2.08 & 48 & 21 & 22 \\
\hline $24-6,127-129$ & 75 & & 60 & 15 & 4 & 17 & 4 & 5 & 2.04 & 1.63 & 52 & 22 & 13 \\
\hline $27-1,127-128$ & 88 & & 80 & 8 & 2 & 10 & & 16 & 1.41 & 1.22 & 50 & 18 & 16 \\
\hline $31-1,100-102$ & 89 & & 80 & 9 & 2 & 9 & & 8 & 2.05 & 0.95 & 65 & 21 & 28 \\
\hline $31-1,124-126$ & 72 & & 62 & 10 & 6 & 20 & 2 & 8 & 2.05 & 0.95 & 54 & 22 & 17 \\
\hline $36-2,72-74$ & 15 & & 10 & 5 & 22 & 64 & & 8 & 2.05 & 0.95 & 42 & 18 & 22 \\
\hline $37-2,95-97$ & 21 & & 15 & 6 & 10 & 40 & 25 & 8 & 2.29 & 1.19 & 48 & 20 & 23 \\
\hline $37-2,130-132$ & 67 & & 55 & 12 & 5 & 22 & 6 & 10 & 2.90 & 1.22 & 105 & 28 & 27 \\
\hline $39-2,38-40$ & 5 & & 5 & & 16 & 69 & 10 & 10 & 2.28 & 1.20 & 50 & 22 & 22 \\
\hline $39-2,145-147$ & 45 & & 20 & 25 & 4 & 31 & 10 & 32 & 1.26 & 0.98 & 110 & 33 & 22 \\
\hline $40-1,9-11$ & 48 & & 40 & 8 & 7 & 30 & 15 & 8 & 1.83 & 1.20 & 50 & 20 & 22 \\
\hline $40-1,33-36$ & 39 & & 30 & 9 & 9 & 42 & 10 & 8 & 2.29 & 1.19 & 54 & 23 & 25 \\
\hline $41-1,2-5$ & 28 & & 20 & 8 & 9 & 43 & 20 & 10 & 2.04 & 0.96 & 60 & 20 & 23 \\
\hline $41-1,10-13$ & 58 & & 45 & 13 & 7 & 25 & 10 & 25 & 2.0 & 0.64 & 90 & 33 & 18 \\
\hline $43-1,13-15$ & 60 & & 50 & 10 & 4 & 25 & 15 & 40 & 1.58 & 0.63 & 150 & 45 & 17 \\
\hline $43-2,82-84$ & 58 & & 40 & $18(?)$ & 4 & 28 & 10 & 25 & 2.58 & 0.38 & 64 & 36 & 25 \\
\hline $43-2,145-148$ & 66 & & 50 & 16 & & 15 & 15 & 16 & 2.58 & 0.60 & 54 & 34 & \\
\hline $43-3,23-26$ & 50 & 2 & 23 & 25 & 3 & 27 & 20 & 32 & 2.24 & 0.49 & - & 43 & 50 \\
\hline $43-3,92-95$ & 53 & & 30 & 22 & 10 & 23 & 15 & 13 & 2.28 & 0.77 & 65 & 23 & \\
\hline $43-3,129-131$ & 65 & & 40 & 25 & 5 & 18 & 12 & 32 & 1.77 & 0.78 & 60 & 40 & 29 \\
\hline $43-4,8-10$ & 65 & & 30 & 35 & 5 & 17 & 13 & 40 & 1.77 & 0.50 & 70 & 45 & 28 \\
\hline $43-4,14-16$ & 65 & 4 & 40 & 21 & 3 & 12 & 20 & 40 & 1.77 & 0.50 & 125 & 39 & 26 \\
\hline $43-5,70-73$ & 73 & & 60 & 13 & 4 & 19 & 4 & 32 & 2.50 & 0.39 & 63 & 38 & 24 \\
\hline $43-5,118-121$ & 71 & & 60 & 11 & 3 & 10 & 16 & & & & & 37 & 21 \\
\hline $43-5,143-146$ & 59 & & 40 & 19 & 7 & 14 & 20 & 25 & 2.24 & 0.51 & 80 & 33 & 26 \\
\hline $44-1,109-111$ & 66 & & 10 & 56 & 7 & 22 & 5 & 25 & 2.58 & 0.38 & & 37 & 27 \\
\hline $44-1,143-145$ & 66 & & 10 & 56 & 2 & 7 & 25 & & & & & 37 & \\
\hline $45-2,49-53$ & 57 & & 40 & 17 & 7 & 23 & 13 & 16 & 2.28 & 0.47 & 70 & 24 & 24 \\
\hline $45-2,55-57$ & 84 & & 5 & 79 & 3 & 3 & 10 & & & & & 29 & 44 \\
\hline $46-5,84-87$ & 60 & & 45 & 15 & 5 & 30 & 5 & 16 & 2.31 & 0.75 & 70 & 30 & 22 \\
\hline $46-6,50-51$ & 63 & & 40 & 23 & 8 & 27 & 2 & 12 & 2.58 & 0.98 & 60 & 27 & 19 \\
\hline $47-1,56-58$ & 8 & & 5 & 3 & 10 & 52 & 27 & 10 & 2.29 & 0.76 & 60 & 22 & 19 \\
\hline $47-6,98-100$ & 7 & & 7 & & 13 & 40 & 39 & 16 & 2.58 & 0.60 & 70 & 30 & 24 \\
\hline $48-2,126-129$ & 11 & & 11 & & 11 & 47 & 30 & 12 & 2.28 & 0.77 & 100 & 25 & 26 \\
\hline $49-4,4-6$ & 30 & & 25 & 5 & 7 & 50 & 13 & 12 & 2.28 & 0.77 & 120 & 25 & 24 \\
\hline $49-4,64-66$ & 13 & & 10 & 3 & 8 & 69 & 10 & & & & & 20 & 21 \\
\hline $51-5,20-21$ & 33 & & 10 & 23 & 5 & 42 & 20 & & & & & 22 & 24 \\
\hline $51-5,67-70$ & 28 & & 20 & 8 & 10 & 52 & 17 & 10 & 2.56 & 0.95 & 200 & 24 & 22 \\
\hline $52-1,136-139$ & 46 & & 36 & 10 & 7 & 43 & 4 & 8 & 2.29 & 1.19 & 140 & 22 & 21 \\
\hline $52-1,139-141$ & 43 & & 30 & 13 & 5 & 37 & 15 & & & & & 20 & 21 \\
\hline $53-1,5-7$ & 19 & & 6 & 13 & 5 & 54 & 12 & 16 & 2.58 & 0.60 & 300 & 24 & 23 \\
\hline $53-1,14-16$ & 8 & & 3 & 5 & 4 & 53 & 25 & 12 & 2.58 & 0.98 & 180 & 24 & 23 \\
\hline $54-3,74-77$ & 36 & & 20 & 16 & 6 & 58 & & & & & & 24 & 33 \\
\hline $54-3,95-98$ & 37 & & 20 & 17 & 6 & 53 & & & & & & 24 & 20 \\
\hline $55-2,78-80$ & 54 & & 44 & 10 & 3 & 43 & & 10 & 2.56 & 0.95 & 200 & 22 & 25 \\
\hline $55-2,87-89$ & 48 & & 35 & 13 & 5 & 44 & & 8 & 2.29 & 1.19 & 140 & 20 & 17 \\
\hline $57-2,32-34$ & 24 & & 20 & 4 & 13 & 63 & & 8 & 2.29 & 1.19 & 60 & 21 & 27 \\
\hline $59-1,14-16$ & 56 & & 30 & 26 & 3 & 36 & & 12 & 2.28 & 0.77 & 200 & 26 & 25 \\
\hline $59-1,19-22$ & 66 & & 45 & 21 & 3 & 41 & & 12 & 2.56 & 0.61 & 130 & 24 & 23 \\
\hline $59-1,81-84$ & 60 & & 30 & 30 & 2 & 33 & & 16 & 2.31 & 0.75 & 170 & 25 & 24 \\
\hline $60-6,148-150$ & 87 & & 70 & 17 & & 11 & 2 & 8 & 2.04 & 1.05 & 50 & 23 & 19 \\
\hline
\end{tabular}


Lithology, Holes 400/400A

\begin{tabular}{|c|c|c|c|c|c|c|c|c|c|c|c|c|c|c|c|c|c|c|c|c|c|c|c|c|c|c|}
\hline $\begin{array}{c}\text { Sample } \\
\text { (Interval in cm) }\end{array}$ & $\mathrm{QR}$ & QRO & $\mathrm{QA}$ & MIC & OMIN & ROC & MUDA & PYR & GLAU & MAN & PFO & PTER & BFO & ECH & OST & LAM & BRY & COR & UNSP & RAD & DIA & SPIC & PLA & FISH & OTH & TOTAL \\
\hline Hole 400 & & & & & & & & & & & Num & ner of $\mathrm{Pa}$ & rticles & & & & & & & & & & & & & \\
\hline $1-1,48 \cdot 50$ & 0 & 1 & 8 & 4 & 3 & 1 & 6 & 71 & 0 & 0 & 536 & 0 & 10 & 0 & 0 & 0 & 0 & 0 & 4 & 16 & 4 & 0 & 2 & 0 & 2 & 668 \\
\hline \multicolumn{27}{|l|}{ Hole $400 \mathrm{~A}$} \\
\hline $1-1,60-62$ & 1 & 2 & 5 & 3 & 0 & 0 & 4 & 0 & 2 & 0 & 541 & 0 & 10 & 0 & 1 & 0 & 0 & 0 & 1 & 8 & 0 & 0 & 0 & 0 & 3 & 581 \\
\hline $1-1,85-87$ & $r_{23}^{5}$ & 9 & $\begin{array}{r}50 \\
307\end{array}$ & 36 & ${ }_{0}^{0}$ & $\frac{1}{2}$ & 14 & 0 & 4 & 0 & 474 & 0 & 10 & 0 & 0 & 0 & 0 & 0 & 3 & 5 & 0 & 0 & 3 & 0 & 0 & 614 \\
\hline $2-2,120-123$ & 23 & $\frac{1}{5}$ & 327 & 27 & 1 & ${ }_{4}^{3}$ & 0 & 1 & 11 & 0 & 160 & 0 & 32 & 0 & 0 & 0 & 0 & 0 & 38 & 0 & 0 & 0 & 1 & 0 & 2 & 627 \\
\hline $\begin{array}{l}2-2,125-126 \\
4-488-89\end{array}$ & $?$ & $\begin{array}{l}5 \\
0\end{array}$ & $\begin{array}{l}187 \\
23\end{array}$ & 13 & $\begin{array}{l}1 \\
2\end{array}$ & 4 & $\begin{array}{r}12 \\
8\end{array}$ & ${ }_{21}^{0}$ & $\begin{array}{l}5 \\
0\end{array}$ & ${ }_{0}^{0}$ & 390 & 0 & 19 & 0 & 0 & 2 & 0 & 0 & 4 & 0 & 0 & 0 & 1 & 1 & 2 & 653 \\
\hline $\begin{array}{l}4-4,88-89 \\
6-2,78-80\end{array}$ & $\begin{array}{l}0 \\
0 \\
0\end{array}$ & $\begin{array}{l}0 \\
0\end{array}$ & $\begin{array}{r}23 \\
3\end{array}$ & $\stackrel{7}{9}$ & ${ }_{0}^{2}$ & $\begin{array}{l}1 \\
0\end{array}$ & $\begin{array}{l}8 \\
0\end{array}$ & $\begin{array}{r}21 \\
2\end{array}$ & $\begin{array}{l}0 \\
0\end{array}$ & $\begin{array}{l}0 \\
0\end{array}$ & $\begin{array}{l}487 \\
562\end{array}$ & $\begin{array}{l}0 \\
0\end{array}$ & $\begin{array}{l}66 \\
13\end{array}$ & $\begin{array}{l}1 \\
0\end{array}$ & $\stackrel{0}{0}$ & $\begin{array}{l}0 \\
0\end{array}$ & $\stackrel{0}{0}$ & $\begin{array}{l}0 \\
0\end{array}$ & ${ }_{2}^{0}$ & $\stackrel{0}{0}$ & $\begin{array}{l}0 \\
0\end{array}$ & $\frac{1}{0}$ & 0 & 2 & 1 & 620 \\
\hline $9-3,20-22$ & 1 & 2 & 140 & 7 & 0 & 1 & 2 & 0 & 10 & 0 & $\begin{array}{l}362 \\
230\end{array}$ & 0 & 119 & 2 & 0 & $\begin{array}{l}0 \\
0\end{array}$ & $\begin{array}{l}0 \\
0\end{array}$ & $\begin{array}{l}0 \\
0\end{array}$ & 62 & ${ }_{0}^{0}$ & $\begin{array}{l}0 \\
0\end{array}$ & $\begin{array}{l}0 \\
0\end{array}$ & ${ }_{0}^{0}$ & 3 & 0 & 594 \\
\hline $10-2,86-87$ & 37 & 4 & 170 & 9 & 0 & i & 6 & 7 & 10 & 0 & 91 & 0 & 92 & 3 & 0 & 0 & 0 & 0 & $\begin{array}{l}62 \\
46\end{array}$ & 0 & $\begin{array}{l}0 \\
0\end{array}$ & $\begin{array}{l}0 \\
2\end{array}$ & $\begin{array}{l}0 \\
0\end{array}$ & $\begin{array}{l}0 \\
1\end{array}$ & ${ }_{8}^{8}$ & $\begin{array}{l}584 \\
487\end{array}$ \\
\hline $13-3,53-55$ & 0 & 1 & 21 & 2 & 0 & 0 & 21 & 16 & 0 & 0 & 461 & 0 & 18 & 1 & 0 & 0 & 0 & 0 & 0 & 0 & 0 & 0 & $\begin{array}{l}0 \\
0\end{array}$ & $\begin{array}{l}1 \\
2\end{array}$ & $\begin{array}{l}8 \\
2\end{array}$ & $\begin{array}{l}487 \\
545\end{array}$ \\
\hline $13-6,60-61$ & 1 & 2 & 12 & 2 & 0 & 0 & 49 & 3 & 0 & 0 & 440 & 0 & 19 & 0 & 0 & 0 & 0 & 0 & 24 & 0 & 0 & 0 & 0 & 3 & ${ }_{3}^{2}$ & $\begin{array}{l}558 \\
558\end{array}$ \\
\hline $13-6,63-64$ & 0 & 0 & 9 & 1 & 1 & 2 & 29 & 2 & 0 & 0 & 311 & & 27 & 0 & 0 & 0 & 0 & 0 & 61 & 0 & 0 & 5 & 0 & 2 & 1 & 451 \\
\hline $\begin{array}{l}14-2,38-40 \\
15-4,75-77\end{array}$ & ${ }_{0}^{1}$ & 0 & $\begin{array}{r}8 \\
14\end{array}$ & ${ }_{0}^{0}$ & 0 & $\begin{array}{l}0 \\
0\end{array}$ & 127 & $\begin{array}{r}0 \\
54\end{array}$ & $\begin{array}{l}0 \\
0\end{array}$ & ${ }_{0}^{0}$ & $\begin{array}{l}482 \\
243\end{array}$ & $\begin{array}{l}0 \\
0\end{array}$ & 11 & ${ }_{2}^{0}$ & $\stackrel{0}{0}$ & $0_{0}^{0}$ & $\begin{array}{l}0 \\
0\end{array}$ & ${ }_{0}^{0}$ & 4 & 0 & 0 & 1 & 0 & 1 & 3 & 518 \\
\hline $\begin{array}{l}15-4,7.75-77 \\
17-1,43-44\end{array}$ & $\begin{array}{l}0 \\
0\end{array}$ & $\begin{array}{l}1 \\
0\end{array}$ & $\begin{array}{l}14 \\
46\end{array}$ & $\begin{array}{l}0 \\
0\end{array}$ & $\begin{array}{l}1 \\
4\end{array}$ & $\begin{array}{l}0 \\
0\end{array}$ & $\begin{array}{l}127 \\
141\end{array}$ & $\begin{array}{l}54 \\
80\end{array}$ & $\begin{array}{l}0 \\
1\end{array}$ & $\begin{array}{l}0 \\
0\end{array}$ & $\begin{array}{r}243 \\
39\end{array}$ & $\begin{array}{l}0 \\
0\end{array}$ & $\begin{array}{l}71 \\
44\end{array}$ & ${ }_{0}^{2}$ & $\begin{array}{l}0 \\
0\end{array}$ & $\begin{array}{l}0 \\
0\end{array}$ & $\begin{array}{l}0 \\
0\end{array}$ & $\begin{array}{l}0 \\
0\end{array}$ & ${ }_{25}^{42}$ & $\begin{array}{l}1 \\
0\end{array}$ & $\begin{array}{l}0 \\
0\end{array}$ & 4 & ${ }_{0}^{0}$ & 13 & 3 & 576 \\
\hline $20-5,132-134$ & 1 & 1 & 107 & 3 & 0 & 0 & 163 & 3 & 3 & 0 & 82 & 5 & 130 & 0 & 0 & 0 & $\begin{array}{l}0 \\
0\end{array}$ & $\begin{array}{l}0 \\
0\end{array}$ & $\begin{array}{l}25 \\
76\end{array}$ & $\begin{array}{l}0 \\
0\end{array}$ & $\begin{array}{l}0 \\
0\end{array}$ & $\frac{1}{7}$ & ${ }_{0}^{0}$ & 26 & 1 & 386 \\
\hline $24-6,127-129$ & 0 & 0 & 1 & 0 & 1 & 0 & 21 & 0 & 0 & 0 & 102 & 319 & 40 & 3 & 0 & 0 & 0 & 0 & $\begin{array}{l}76 \\
24\end{array}$ & $\begin{array}{l}0 \\
0\end{array}$ & $\begin{array}{l}0 \\
0\end{array}$ & ${ }_{3}^{7}$ & $\begin{array}{l}0 \\
0\end{array}$ & $\begin{array}{l}26 \\
10\end{array}$ & ${ }_{2}^{7}$ & $\begin{array}{l}614 \\
526\end{array}$ \\
\hline $27-1,127-128$ & 0 & 0 & 10 & 1 & 1 & 0 & 56 & 0 & 1 & 0 & 230 & 2 & 54 & 0 & 0 & 0 & 0 & 0 & 120 & 0 & 0 & 2 & 2 & $\begin{array}{r}10 \\
7\end{array}$ & $\frac{2}{4}$ & $\begin{array}{l}526 \\
490\end{array}$ \\
\hline $31-1,100-102$ & ${ }_{0}^{0}$ & 0 & 0 & 0 & ${ }_{0}^{0}$ & 0 & 21 & 0 & 0 & 0 & 390 & 147 & 10 & 0 & 0 & 0 & 0 & 0 & 28 & 3 & 0 & ii & $i$ & 4 & 5 & 620 \\
\hline & $\frac{0}{2}$ & 0 & $10^{3}$ & $\begin{array}{l}0 \\
6\end{array}$ & $\stackrel{0}{2}_{2}^{2}$ & 0 & $\begin{array}{l}32 \\
38\end{array}$ & $\begin{array}{l}1 \\
5\end{array}$ & $\begin{array}{c}0 \\
5\end{array}$ & $\stackrel{0}{0}$ & $\begin{array}{r}205 \\
83\end{array}$ & 128 & $\begin{array}{l}48 \\
56\end{array}$ & 10 & $0_{0}^{0}$ & ${ }_{0}^{0}$ & 0 & 0 & 30 & 11 & 0 & 18 & 0 & 1 & 2 & 489 \\
\hline $\begin{array}{l}36-2,72-74 \\
37-2,95-97\end{array}$ & ${ }_{0}^{2}$ & $\begin{array}{l}1 \\
0\end{array}$ & $\begin{array}{r}170 \\
10\end{array}$ & $\begin{array}{l}6 \\
2\end{array}$ & ${ }_{0}^{2}$ & $l_{0}^{1}$ & $\begin{array}{r}38 \\
253\end{array}$ & $\begin{array}{l}5 \\
1\end{array}$ & $\begin{array}{l}5 \\
1\end{array}$ & $\begin{array}{l}0 \\
0\end{array}$ & $\begin{array}{l}83 \\
15\end{array}$ & 9 & $\begin{array}{l}56 \\
55\end{array}$ & ${ }_{2}^{3}$ & $\begin{array}{l}0 \\
0\end{array}$ & $\begin{array}{l}0 \\
0\end{array}$ & $\begin{array}{l}0 \\
0\end{array}$ & $\begin{array}{l}0 \\
0\end{array}$ & 129 & $\begin{array}{r}1 \\
49\end{array}$ & ${ }_{0}^{0}$ & $23^{3}$ & 1 & 44 & 8 & $\begin{array}{l}567 \\
775\end{array}$ \\
\hline $37-2,130-132$ & 0 & 0 & 20 & 1 & 0 & 0 & $\begin{aligned} 253 \\
2\end{aligned}$ & 0 & 1 & $\begin{array}{l}0 \\
0\end{array}$ & $\begin{array}{l}15 \\
71\end{array}$ & ${ }_{0}^{2}$ & 348 & ${ }_{1}^{2}$ & $\begin{array}{l}0 \\
0\end{array}$ & $\begin{array}{l}0 \\
0\end{array}$ & $\begin{array}{l}0 \\
0\end{array}$ & $\begin{array}{l}0 \\
0\end{array}$ & $\begin{array}{l}36 \\
57\end{array}$ & $\begin{array}{r}49 \\
3\end{array}$ & ${ }_{0}^{0}$ & $\begin{array}{l}281 \\
17\end{array}$ & $\begin{array}{l}0 \\
0\end{array}$ & $\begin{array}{c}41 \\
1\end{array}$ & 4 & $\begin{array}{l}752 \\
593\end{array}$ \\
\hline $39-2,145-147$ & 0 & 0 & 19 & 9 & 1 & 2 & 6 & 0 & 1 & 0 & 67 & 0 & 111 & 0 & 0 & 0 & 0 & 0 & 213 & 1 & $\begin{array}{l}0 \\
0\end{array}$ & $\begin{array}{r}17 \\
132\end{array}$ & $\begin{array}{l}0 \\
0\end{array}$ & $\begin{array}{l}1 \\
0\end{array}$ & 1 & \\
\hline $40-1,9-11$ & 0 & 1 & 126 & 1 & 2 & 0 & 84 & 0 & 11 & 0 & 6 & 0 & 41 & 1 & 0 & 0 & 0 & 0 & 0 & 13 & 0 & 248 & 0 & 7 & ${ }_{3}^{1}$ & $\begin{array}{l}563 \\
544\end{array}$ \\
\hline $40-1,33-36$ & 0 & 0 & 10 & 1 & 0 & 0 & 54 & 0 & 0 & 2 & 24 & 0 & 81 & 1 & 0 & 0 & 0 & 0 & 91 & 54 & 0 & 128 & 0 & 19 & 1 & 466 \\
\hline $\begin{array}{l}41-1,2-5 \\
41-1,0-13\end{array}$ & ${ }_{0}^{0}$ & $\begin{array}{l}0 \\
0\end{array}$ & 6 & 3 & $\stackrel{0}{0}$ & $\begin{array}{l}0 \\
0\end{array}$ & 117 & $\begin{array}{l}0 \\
0\end{array}$ & 0 & 0 & 4 & 0 & 35 & 1 & ${ }_{0}^{0}$ & 0 & 0 & $\stackrel{0}{0}$ & 46 & 83 & 2 & 190 & 0 & 19 & 5 & 511 \\
\hline $\begin{array}{l}41-1,10-13 \\
43-1,10-12\end{array}$ & $\begin{array}{l}0 \\
0\end{array}$ & $\begin{array}{l}0 \\
0\end{array}$ & $\begin{array}{l}11 \\
10\end{array}$ & ${ }_{1}^{3}$ & $\begin{array}{l}0 \\
1\end{array}$ & ${ }_{0}^{0}$ & 99 & $\begin{array}{l}0 \\
0\end{array}$ & 1 & $\begin{array}{l}0 \\
0\end{array}$ & $\begin{array}{l}84 \\
65\end{array}$ & $\begin{array}{l}0 \\
0\end{array}$ & $\begin{array}{l}273 \\
292\end{array}$ & $\begin{array}{l}0 \\
1\end{array}$ & $\stackrel{0}{0}$ & $\begin{array}{l}0 \\
0\end{array}$ & $\begin{array}{l}0 \\
0\end{array}$ & $\begin{array}{l}0 \\
0\end{array}$ & $\begin{array}{l}47 \\
85\end{array}$ & 17 & 0 & $\begin{array}{r}80 \\
126\end{array}$ & ${ }_{0}^{0}$ & 1 & ${ }_{4}^{2}$ & $\begin{array}{l}528 \\
606\end{array}$ \\
\hline $43-2,82-84$ & 0 & 0 & 18 & 0 & 1 & $\begin{array}{l}0 \\
0\end{array}$ & $\begin{array}{l}13 \\
36\end{array}$ & 0 & 0 & 0 & $\begin{array}{l}63 \\
18\end{array}$ & $\begin{array}{l}0 \\
0\end{array}$ & $\begin{array}{l}292 \\
300\end{array}$ & $\begin{array}{l}1 \\
0\end{array}$ & $\begin{array}{l}0 \\
0\end{array}$ & ${ }_{0}^{0}$ & $\begin{array}{l}0 \\
0\end{array}$ & $\begin{array}{l}0 \\
0\end{array}$ & $\begin{array}{l}85 \\
76\end{array}$ & $\begin{array}{l}4 \\
5\end{array}$ & $\frac{1}{1}$ & $\begin{array}{l}126 \\
100\end{array}$ & $\begin{array}{l}0 \\
0\end{array}$ & $\frac{2}{3}$ & 4 & 606 \\
\hline $43-2,145 \cdot 148$ & 0 & 0 & 8 & 3 & 2 & 0 & 37 & 0 & 0 & 0 & 36 & 0 & 281 & 0 & 0 & 0 & $\begin{array}{l}0 \\
0\end{array}$ & $\begin{array}{l}0 \\
0\end{array}$ & $\begin{array}{l}76 \\
54\end{array}$ & $\begin{array}{l}5 \\
8\end{array}$ & $l_{0}^{1}$ & $\begin{array}{l}100 \\
245\end{array}$ & $\begin{array}{l}0 \\
0\end{array}$ & ${ }_{1}^{3}$ & ${ }_{2}^{7}$ & $\begin{array}{l}565 \\
677\end{array}$ \\
\hline $43-3,23-26$ & 0 & 0 & 21 & 3 & 0 & 0 & 6 & 0 & 0 & 0 & 21 & 0 & 307 & 1 & 0 & 0 & 0 & 0 & 101 & 3 & 0 & 45 & 0 & $i$ & $0_{0}^{2}$ & $\begin{array}{l}677 \\
509\end{array}$ \\
\hline $43-3,92-95$ & 0 & 1 & 48 & 2 & 1 & 1 & 23 & 0 & 0 & 0 & 5 & 0 & 105 & 0 & 0 & 0 & 0 & 0 & 35 & 13 & 0 & 285 & 1 & 3 & $\begin{array}{l}0 \\
0\end{array}$ & $\begin{array}{l}509 \\
523\end{array}$ \\
\hline $43-3,129-131$ & 0 & 0 & 2 & 1 & 2 & 0 & 24 & 0 & 0 & 0 & 24 & 0 & 204 & 0 & 0 & 0 & 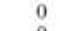 & 0 & 44 & 3 & 0 & 164 & 0 & 4 & 0 & 472 \\
\hline $43-4,8-10$ & 0 & 0 & 39 & 5 & 2 & 0 & 8 & 0 & 0 & ${ }_{0}^{0}$ & 6 & 0 & 92 & ${ }_{0}^{0}$ & 0 & 0 & 0 & 0 & 75 & 3 & 0 & 263 & 0 & 4 & 2 & 499 \\
\hline $\begin{array}{l}43-4,14 \cdot 16 \\
43-5,70-73\end{array}$ & $\begin{array}{l}0 \\
0\end{array}$ & $\begin{array}{l}0 \\
0\end{array}$ & $\begin{array}{l}13 \\
36\end{array}$ & ${ }_{2}^{2}$ & $\frac{1}{2}$ & $\begin{array}{l}0 \\
0\end{array}$ & 37 & ${ }_{0}^{0}$ & $\begin{array}{l}0 \\
0\end{array}$ & $\begin{array}{l}0 \\
0\end{array}$ & $\begin{array}{r}14 \\
2\end{array}$ & $\begin{array}{l}0 \\
0 \\
0\end{array}$ & $\begin{array}{l}262 \\
81\end{array}$ & $\begin{array}{l}0 \\
0\end{array}$ & ${ }_{0}^{0}$ & $\begin{array}{l}0 \\
0\end{array}$ & $\begin{array}{l}0 \\
0\end{array}$ & $\begin{array}{l}0 \\
0 \\
0\end{array}$ & $\begin{array}{l}51 \\
78\end{array}$ & 28 & ${ }_{0}^{0}$ & $\begin{array}{r}86 \\
230\end{array}$ & $\begin{array}{l}0 \\
0\end{array}$ & $\begin{array}{l}0 \\
3\end{array}$ & 0 & $\begin{array}{l}435 \\
488\end{array}$ \\
\hline $\begin{array}{l}43-5,70.73 \\
43-5,118-121\end{array}$ & $\begin{array}{l}0 \\
0\end{array}$ & $\begin{array}{l}0 \\
0\end{array}$ & $\begin{array}{r}36 \\
4\end{array}$ & $\frac{2}{3}$ & ${ }_{1}^{2}$ & $\begin{array}{l}0 \\
0\end{array}$ & $\begin{array}{r}37 \\
101\end{array}$ & $\begin{array}{l}0 \\
0\end{array}$ & $\begin{array}{l}0 \\
0\end{array}$ & $\begin{array}{l}0 \\
0\end{array}$ & ${ }_{8}^{2}$ & $\begin{array}{l}0 \\
0\end{array}$ & $\begin{array}{r}81 \\
157\end{array}$ & $\begin{array}{l}0 \\
0\end{array}$ & $\begin{array}{l}0 \\
0\end{array}$ & $\begin{array}{l}0 \\
0\end{array}$ & $\begin{array}{l}0 \\
0\end{array}$ & $\begin{array}{l}0 \\
0\end{array}$ & $\begin{array}{r}78 \\
121\end{array}$ & $\begin{array}{r}16 \\
0\end{array}$ & $\begin{array}{l}0 \\
0\end{array}$ & $\begin{array}{l}230 \\
79\end{array}$ & $\begin{array}{l}0 \\
0\end{array}$ & ${ }_{0}^{3}$ & 1 & 488 \\
\hline $43-5,143-146$ & 2 & 1 & 74 & 1 & 4 & 0 & 5 & 0 & 4 & 0 & 57 & 0 & 78 & 0 & $\begin{array}{l}0 \\
0\end{array}$ & $\begin{array}{l}0 \\
0\end{array}$ & $\begin{array}{l}0 \\
0\end{array}$ & $\begin{array}{l}0 \\
0\end{array}$ & $\begin{array}{r}121 \\
66\end{array}$ & & $\begin{array}{l}0 \\
0\end{array}$ & $\begin{array}{r}79 \\
223\end{array}$ & $\begin{array}{l}0 \\
1\end{array}$ & 0 & $\begin{array}{l}0 \\
4\end{array}$ & $\begin{array}{l}474 \\
528\end{array}$ \\
\hline $44-1,109-111$ & 0 & 0 & 4 & i & 1 & 0 & 21 & 0 & 0 & 0 & 21 & 0 & 93 & 1 & 0 & 0 & 0 & 0 & 141 & $i$ & 0 & 189 & 0 & 1 & 4 & $\begin{array}{l}528 \\
479\end{array}$ \\
\hline $44-1,143-145$ & 0 & 0 & 13 & 2 & 0 & 0 & 77 & 0 & 0 & 0 & 3 & 0 & 64 & 0 & 0 & 0 & 0 & 0 & 217 & 2 & 0 & 75 & 0 & 0 & 4 & $\begin{array}{l}479 \\
457\end{array}$ \\
\hline & 1 & 0 & 34 & 3 & 4 & 2 & 1 & 0 & 1 & 0 & 10 & 0 & 47 & 0 & 0 & 0 & 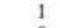 & 0 & 58 & 3 & 0. & 391 & 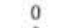 & 3 & 3 & $\begin{array}{l}451 \\
571\end{array}$ \\
\hline $45-2,55-57$ & 0 & 1 & 8 & 5 & 0 & 0 & 45 & 0 & 1 & 0 & 9 & 0 & 57 & 0 & 0 & 0 & 0 & 0 & 194 & 1 & 0 & 169 & 0 & 0 & 3 & 493 \\
\hline $\begin{array}{l}46-5,84-87 \\
46-6,50-51\end{array}$ & 8 & 4 & $\begin{array}{l}317 \\
190\end{array}$ & 3 & ${ }_{1}^{2}$ & $\begin{array}{l}0 \\
0\end{array}$ & 39 & ${ }_{0}^{0}$ & 13 & $\begin{array}{l}0 \\
0\end{array}$ & 1 & $\begin{array}{l}0 \\
0\end{array}$ & 41 & 8 & 0 & 0 & 0 & ${ }_{0}^{0}$ & 108 & 2 & 0 & 52 & 0 & 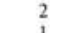 & 1 & 495 \\
\hline $\begin{array}{l}46-6,50-51 \\
47-1,56-58\end{array}$ & $\begin{array}{l}3 \\
2\end{array}$ & $\begin{array}{l}1 \\
2\end{array}$ & $\begin{array}{r}190 \\
90\end{array}$ & ${ }_{0}^{3}$ & $\begin{array}{l}1 \\
3\end{array}$ & $\begin{array}{l}0 \\
0\end{array}$ & ${ }^{3}$ & $\begin{array}{l}0 \\
1\end{array}$ & $\begin{array}{r}13 \\
3\end{array}$ & $\begin{array}{l}0 \\
0\end{array}$ & 1 & $\begin{array}{l}0 \\
0 \\
0\end{array}$ & 16 & $\begin{array}{l}8 \\
0\end{array}$ & $\begin{array}{l}0 \\
0\end{array}$ & $\begin{array}{l}0 \\
0\end{array}$ & 0 & $\begin{array}{l}0 \\
0\end{array}$ & $\begin{array}{r}108 \\
20\end{array}$ & $\begin{array}{l}0 \\
56\end{array}$ & $\begin{array}{l}0 \\
0\end{array}$ & 9 & 0 & $\begin{array}{l}1 \\
0\end{array}$ & 15 & $\begin{array}{l}513 \\
557\end{array}$ \\
\hline $\begin{array}{l}4-1,56-38 \\
47-6,98-100\end{array}$ & 0 & 0 & 15 & 0 & 0 & 0 & $\begin{array}{l}64 \\
14\end{array}$ & $\begin{array}{l}1 \\
0\end{array}$ & $\begin{array}{l}3 \\
0\end{array}$ & $\begin{array}{r}0 \\
35\end{array}$ & $\begin{array}{l}1 \\
0\end{array}$ & $\begin{array}{l}0 \\
0\end{array}$ & $\begin{array}{r}16 \\
6\end{array}$ & $\begin{array}{l}0 \\
0\end{array}$ & $\begin{array}{l}0 \\
0\end{array}$ & ${ }_{0}^{0}$ & $\begin{array}{l}0 \\
0\end{array}$ & $\begin{array}{l}0 \\
0\end{array}$ & $\begin{array}{l}20 \\
20\end{array}$ & $\begin{array}{l}256 \\
229\end{array}$ & $\begin{array}{l}0 \\
0\end{array}$ & 89 & ${ }_{0}^{0}$ & 0 & ${ }_{1}^{10}$ & $\begin{array}{l}557 \\
541\end{array}$ \\
\hline 6-129 & 3 & 3 & 37 & 1 & 4 & 0 & 3 & 0 & 3 & 0 & 0 & 0 & $\begin{array}{l}0 \\
4\end{array}$ & 0 & 0 & 0 & 0 & 0 & $\begin{array}{r}20 \\
4\end{array}$ & $\begin{array}{l}229 \\
376\end{array}$ & $\begin{array}{l}0 \\
0\end{array}$ & $\begin{array}{r}221 \\
69\end{array}$ & 0 & $\begin{array}{l}0 \\
1\end{array}$ & $\begin{array}{l}1 \\
5\end{array}$ & $\begin{array}{l}541 \\
513\end{array}$ \\
\hline 49 & 0 & 0 & 2 & 1 & 0 & 0 & 29 & 0 & 0 & 0 & 12 & 0 & 14 & 0 & 0 & 0 & 0 & 0 & 47 & 314 & 0 & 39 & 0 & 0 & 4 & $\begin{array}{l}313 \\
462\end{array}$ \\
\hline & 0 & 1 & 9 & 1 & 2 & 0 & 119 & 0 & 0 & 1 & 6 & 0 & 19 & 0 & 0 & 0 & 0 & 0 & 56 & 2 & 0 & 31 & 0 & 0 & 3 & 485 \\
\hline 5 & 0 & 0 & 11 & 2 & 0 & 0 & 324 & 0 & 0 & 0 & 1 & $\begin{array}{l}0 \\
0\end{array}$ & II & ${ }_{0}^{0}$ & ${ }_{0}^{0}$ & 0 & 0 & 0 & 33 & & 0 & . & 0 & 2 & 2 & 479 \\
\hline $\begin{array}{l}51-5,67-70 \\
52-1,136-139\end{array}$ & $\begin{array}{l}0 \\
0\end{array}$ & $\begin{array}{l}0 \\
0 \\
0\end{array}$ & $\frac{5}{3}$ & 0 & ${ }_{0}^{3}$ & $\begin{array}{l}0 \\
0\end{array}$ & $\begin{array}{l}20 \\
10\end{array}$ & $\begin{array}{l}0 \\
0\end{array}$ & $\begin{array}{l}0 \\
0\end{array}$ & $\begin{array}{l}0 \\
0\end{array}$ & 10 & $\begin{array}{l}0 \\
0\end{array}$ & $\begin{array}{ll}11 \\
8\end{array}$ & $0_{0}^{0}$ & ${ }_{0}^{0}$ & $\begin{array}{l}0 \\
0\end{array}$ & 0 & ${ }_{0}^{0}$ & 14 & 484 & $0_{0}^{0}$ & 44 & 0 & 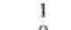 & 1 & 593 \\
\hline $\begin{array}{l}52-1,136-139 \\
52-1,139-141\end{array}$ & $\begin{array}{l}0 \\
0\end{array}$ & $\begin{array}{l}0 \\
0\end{array}$ & $\begin{array}{l}3 \\
6\end{array}$ & i & $\begin{array}{l}0 \\
1\end{array}$ & $\begin{array}{l}0 \\
0\end{array}$ & $\begin{array}{r}10 \\
240\end{array}$ & $\begin{array}{l}0 \\
0\end{array}$ & $\begin{array}{l}0 \\
1\end{array}$ & ${ }_{0}^{0}$ & $\begin{array}{r}8 \\
13\end{array}$ & $\begin{array}{l}0 \\
0 \\
0\end{array}$ & 22 & $\begin{array}{l}0 \\
0\end{array}$ & $\begin{array}{l}0 \\
0\end{array}$ & $\begin{array}{l}0 \\
0\end{array}$ & ${ }_{0}^{0}$ & ${ }_{0}^{0}$ & 8 & $\begin{array}{l}483 \\
267\end{array}$ & ${ }_{0}^{0}$ & $\begin{array}{l}17 \\
22\end{array}$ & 0 & $0_{0}^{0}$ & ${ }_{4}^{0}$ & 538 \\
\hline $\begin{array}{l}52-1,1,5 \\
53-1,5\end{array}$ & $\begin{array}{l}0 \\
1\end{array}$ & $\begin{array}{l}0 \\
1\end{array}$ & 25 & $i$ & $\begin{array}{l}1 \\
0\end{array}$ & $\begin{array}{l}0 \\
1\end{array}$ & $\begin{array}{r}240 \\
31\end{array}$ & $\begin{array}{l}0 \\
0\end{array}$ & 1 & $\begin{array}{l}0 \\
0\end{array}$ & $\begin{array}{l}13 \\
23\end{array}$ & $\begin{array}{l}0 \\
0\end{array}$ & $\begin{array}{r}22 \\
4\end{array}$ & $\begin{array}{l}0 \\
0\end{array}$ & $\begin{array}{l}0 \\
0\end{array}$ & $\begin{array}{l}0 \\
0\end{array}$ & $\begin{array}{l}0 \\
0\end{array}$ & $\begin{array}{l}0 \\
0\end{array}$ & $\begin{array}{l}41 \\
14\end{array}$ & $\begin{array}{l}267 \\
417\end{array}$ & $\begin{array}{l}0 \\
0\end{array}$ & $\begin{array}{l}22 \\
16\end{array}$ & $\begin{array}{l}0 \\
0\end{array}$ & $\begin{array}{l}0 \\
0\end{array}$ & 4 & $\begin{array}{l}618 \\
538\end{array}$ \\
\hline & 0 & 0 & 2 & 0 & 1 & 0 & 31 & 0 & $\begin{array}{l}1 \\
2\end{array}$ & ${ }_{0}^{0}$ & 1 & 0 & 0 & 0 & 0 & 0 & 0 & 0 & $\begin{array}{r}14 \\
0\end{array}$ & & $\begin{array}{l}0 \\
0\end{array}$ & 8 & 0 & 0 & 0 & $\begin{array}{l}338 \\
445\end{array}$ \\
\hline 5 & 0 & 0 & 1 & 0 & 2 & 0 & 228 & 0 & 0 & 0 & 3 & 0 & 0 & 0 & 0 & 0 & 0 & 0 & 2 & & 0 & 6 & 0 & 0 & 0 & 412 \\
\hline & 0 & 0 & 2 & 1 & 1 & 0 & 145 & 0 & 0 & 0 & 1 & 0 & 0 & 0 & 0 & 0 & 0 & 0 & 0 & & 0 & 3 & 0 & 0 & 0 & 470 \\
\hline $\begin{array}{l}55-2,7 \\
55,2,8\end{array}$ & 0 & 0 & 3 & 0 & 5 & 0 & 6 & 0 & 0 & 0 & 74 & ${ }_{0}^{0}$ & 6 & 0 & 0 & 0 & 0 & 0 & 0 & 371 & 0 & 0 & 0 & 1 & 2 & 468 \\
\hline $55-2,87$ & 0 & 0 & $\frac{1}{4}$ & $\frac{1}{5}$ & 1 & ${ }_{0}^{0}$ & 0 & $0_{0}^{0}$ & 0 & 0 & $\begin{array}{r}44 \\
447\end{array}$ & 0 & ${ }_{18}^{3}$ & 0 & ${ }_{2}^{0}$ & 0 & 0 & 0 & 6 & 492 & 0 & 1 & 0 & 0 & 3 & 552 \\
\hline $\begin{array}{l}57 \\
59 \\
59\end{array}$ & $\begin{array}{l}0 \\
0\end{array}$ & $\begin{array}{l}0 \\
0\end{array}$ & ${ }_{1}^{4}$ & $\begin{array}{l}5 \\
1\end{array}$ & 1 & $\begin{array}{l}0 \\
0\end{array}$ & $\begin{array}{l}32 \\
10\end{array}$ & $\begin{array}{l}0 \\
0\end{array}$ & $2_{0}^{2}$ & 0 & $\begin{array}{r}447 \\
25\end{array}$ & ${ }_{0}^{0}$ & $\begin{array}{r}18 \\
2\end{array}$ & ${ }_{0}^{1}$ & ${ }_{0}^{2}$ & $\begin{array}{l}0 \\
0\end{array}$ & $\begin{array}{l}0 \\
0\end{array}$ & $0_{0}^{0}$ & 5 & $\begin{array}{r}0 \\
373\end{array}$ & $0_{0}^{0}$ & 0 & ${ }_{0}^{0}$ & 6 & 0 & 523 \\
\hline $\begin{array}{l}59-1,14 \\
59-1,19\end{array}$ & $\begin{array}{l}0 \\
0\end{array}$ & $\begin{array}{l}0 \\
0\end{array}$ & $\frac{1}{1}$ & $\begin{array}{l}1 \\
1\end{array}$ & $\begin{array}{l}1 \\
2\end{array}$ & $\begin{array}{l}0 \\
0\end{array}$ & $\begin{array}{l}100 \\
21\end{array}$ & ${ }_{0}^{0}$ & $\begin{array}{l}0 \\
0\end{array}$ & $\stackrel{0}{0}$ & $\begin{array}{l}25 \\
11\end{array}$ & $\begin{array}{l}0 \\
0\end{array}$ & ${ }_{13}^{2}$ & $\begin{array}{l}0 \\
1\end{array}$ & $\begin{array}{l}0 \\
0\end{array}$ & $\begin{array}{l}0 \\
0\end{array}$ & $\begin{array}{l}0 \\
0\end{array}$ & $\begin{array}{l}0 \\
0\end{array}$ & $\begin{array}{l}0 \\
0\end{array}$ & $\begin{array}{l}373 \\
386\end{array}$ & ${ }_{0}^{0}$ & ${ }_{0}^{0}$ & $\begin{array}{l}0 \\
0 \\
0\end{array}$ & ${ }_{2}^{3}$ & $\begin{array}{l}0 \\
0\end{array}$ & $\begin{array}{l}416 \\
438\end{array}$ \\
\hline $59-1,81-84$ & 0 & 0 & 4 & 0 & 1 & 0 & 27 & 0 & 0 & $\begin{array}{l}0 \\
0\end{array}$ & ${ }_{1}^{1}$ & 0 & 6 & 0 & 0 & 0 & 0 & 0 & 2 & 351 & $\begin{array}{l}0 \\
0\end{array}$ & $\begin{array}{l}0 \\
1\end{array}$ & 0 & $\begin{array}{l}2 \\
1\end{array}$ & 20 & $\begin{array}{l}438 \\
414\end{array}$ \\
\hline $60-6,148-150$ & 0 & 0 & 3 & 1 & 3 & 0 & 10 & 0 & 0 & 0 & 152 & 0 & 40 & 0 & 0 & 0 & 0 & 0 & 304 & 4 & 0 & 0 & 0 & 0 & 1 & 518 \\
\hline
\end{tabular}


TABLE 5C - Continued

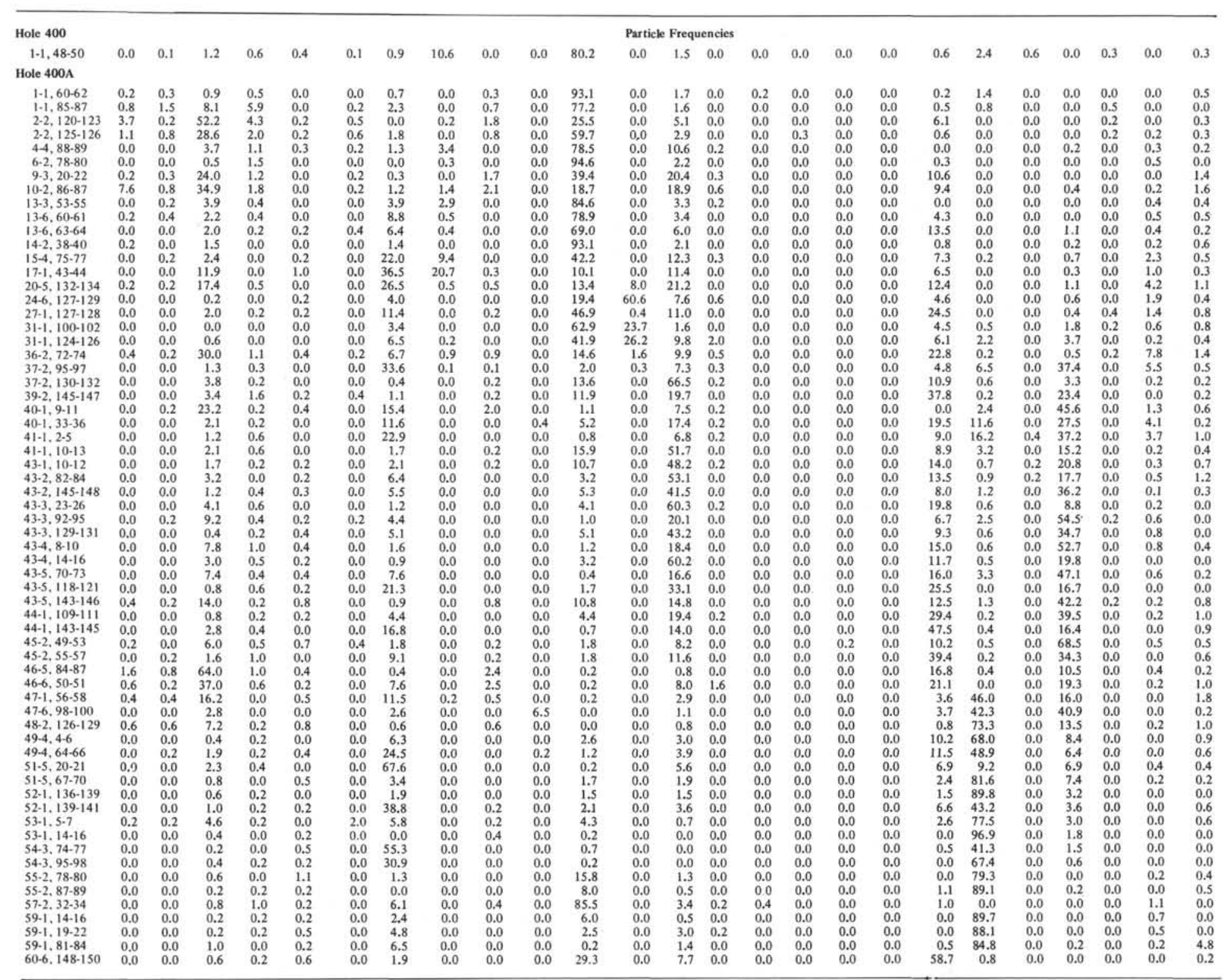


TABLE 5C - Continued

Sample
(Interval in
(n) Hole 400 Terrigenous

Authigenic Calcareous Siliceous Miscellaneous

$1-1,48-50$

\begin{tabular}{llll} 
(\%) & (\%) & (5) & (\%) \\
\hline & 82.34 & 2.99 & 0.60
\end{tabular}

$\begin{array}{llll}0.34 & 95.18 & 1.38 & 0.52 \\ 0.65 & 79.32 & 0.81 & 0.49 \\ 1.91 & 36.68 & 0.00 & 0.48\end{array}$

\begin{tabular}{rr}
2.58 \\
$-1.85-87$ & 18.73 \\
\hline & -1.93
\end{tabular}

$2-2,120-123$
$2-2,125-126$

$4-4,88-89$

$9-3,20-22$
$10-2,86-87$

13-3, $53-55$

$13-6,60-61$
$13-6,63-64$

$15-4,75-77$

$17-1,43-44$

$24-6,127-129$

$27-1,127-128$
$31-1,100-102$

31-1, 124-12

$37-2,95-97$
$37-2,130-132$

$39-2,145-147$

$40-1,33-36$

$41-1,2-5$

$41-1,10-13$
$43-1,10-12$

43-2, $145-148$

$43-3,23-26$
$43-3,-92.95$

$43-3,129-13$

$434,8-10$

$434,14-16$

43-5. 70-73

43-5, $143-146$

44-1, 109-111

45-2, 49-53

$46-5,84-87$

$46-6,50-51$
$47-1,56-58$

$47-6,98-100$
$48-2,126-129$

$49-4,4-6$

$49-4,64-66$
$51-5,20-21$

$52-1,136-139$

$52-1,139-141$

$53-1,5-7,14$

$53-1,14-16$
$54-3,74-77$

$54-3,95-98$
$55-2,78-80$

$55-2,87-89$
$57-2,32-34$

$59-1,14-16$

$59-1,19-22$
$59-1,81-84$

$\begin{array}{ll}63.55 & 0.00 \\ 89.35 & 0.16\end{array}$

$\begin{array}{lll}9.14 & 0.00 & 0.51 \\ 0.72 & 0.00 & 1.37 \\ 4.64 & 0.41 & 1.85\end{array}$

$2.02 \quad 0.34$

$\begin{array}{rr}46.61 & 3.49 \\ 8.26 & 2.94 \\ 11.83 & 0.54\end{array}$

$\begin{array}{ll}9.31 & 0.44 \\ 3 & 0.09\end{array}$

24.83

0.44
0.00
9.38

$\begin{array}{ll}88.07 & 0.00 \\ 86.56 & 0.00\end{array}$

88.47
95.95

0.00
1.11
0.19
0.87

1.08
0.67

44.79
4.37
13.88
3.39

0.98
0.00

47.72
92.78

92.78
82.86
92.74

1.14
0.57

\begin{tabular}{ll}
.14 & 5.37 \\
.57 & 2.28 \\
\hline
\end{tabular}

Note: Terrigenous: $Q R=$ reddish quartz, $Q R O=$ rounded quartz, $Q A=$ angulous quartz
$M I C=$ mica, OMIN $=$ other minerals, $R O C=$ rock fragments, $M U D A=$ muddy

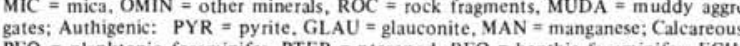

PFO = planktonic foraminifer, PTER = pteropod, BFO = benthic foraminifer, ECH

echinoid, OST = ostracode, $\mathrm{LAM}=$ lamellibranch, $B R Y=$ bryozoan, $C O R=$ coral
UNSP $=$ unspecified carbonate: Siliceous: $R A D=$ tadiolarian, DIA $=$ diatom, SPIC

sponge spiculae; Miscellaneous: PLA = plant remains, $\mathrm{FISH}=$ fish remains, $\mathrm{OTH}$ 
TABLE 6

Summary Of Textural Data

\begin{tabular}{|c|c|c|c|c|c|c|c|c|c|c|c|c|c|c|c|c|c|c|c|c|c|}
\hline \multirow[b]{2}{*}{ Period } & \multicolumn{5}{|c|}{ SITE 400} & \multirow[b]{2}{*}{$\begin{array}{l}\text { Cur- } \\
\text { rent }\end{array}$} & \multirow[b]{2}{*}{$\begin{array}{l}\text { Disso- } \\
\text { lution }\end{array}$} & \multicolumn{7}{|c|}{ SITE 401} & \multicolumn{7}{|c|}{ SITE 402} \\
\hline & $\mathrm{Md}_{1}$ & $\mathrm{Md}_{2}$ & $\mathrm{v}_{1}$ & $\mathrm{v}_{2}$ & I & & & $\mathrm{Md}_{1}$ & $\mathrm{Md}_{2}$ & $\mathrm{v}_{1}$ & $\mathrm{v}_{2}$ & $r$ & $\begin{array}{l}\text { Cur- } \\
\text { rent }\end{array}$ & $\begin{array}{l}\text { Disso- } \\
\text { lution }\end{array}$ & $\mathrm{Md}_{1}$ & $\mathrm{Md}_{2}$ & $\mathrm{v}_{1} \mathrm{Q}$ & $\mathrm{v}_{2}$ & I & $\begin{array}{l}\text { Cur- } \\
\text { rent }\end{array}$ & $\begin{array}{l}\text { Disso- } \\
\text { lution }\end{array}$ \\
\hline $\begin{array}{l}\text { Lower } \\
\text { Pliocene }\end{array}$ & 24 & 21 & 8 & 19 & 0.65 & High & Low & & & & & & & & & & & & & & \\
\hline $\begin{array}{l}\text { Mid-late } \\
\text { Miocene }\end{array}$ & 20 & 20 & 9 & 27 & -0.11 & Low & High & & & & & & & & & & & & & & \\
\hline Early Miocene & 25 & 23 & 23 & 11 & -0.31 & $\begin{array}{l}\text { Moder- } \\
\text { ate }\end{array}$ & $\begin{array}{l}\text { High } \\
\text { to } \\
\text { moder- } \\
\text { ate }\end{array}$ & & & & & & & & & & & & & & \\
\hline Oligocene & 36 & 26 & 19 & 25 & -0.09 & $\begin{array}{l}\text { Very } \\
\text { high } \\
\text { to } \\
\text { moder- } \\
\text { ate }\end{array}$ & $\begin{array}{l}\text { Moder- } \\
\text { ate }\end{array}$ & & & & & & & & & & & & & & \\
\hline Late Eocene & & & & & & & & & & & & & & & 22 & 18 & 4 & 5 & 0.06 & $\begin{array}{c}\text { Low } \\
\text { moder- } \\
\text { ate }\end{array}$ & $\begin{array}{l}\text { Moder- } \\
\text { ate } \\
\text { Low }\end{array}$ \\
\hline $\begin{array}{l}\text { Middle } \\
\text { Eocene }\end{array}$ & 23 & 24 & 13 & 10 & 0.60 & $\begin{array}{l}\text { Moder- } \\
\text { ate } \\
\text { to } \\
\text { high }\end{array}$ & Low & 27 & 20 & 11 & 6 & 0.18 & Low & Low & & & & & & & \\
\hline $\begin{array}{l}\text { Early } \\
\text { Eocene }\end{array}$ & 23 & 24 & 7 & 23 & 0.51 & $\begin{array}{l}\text { Moder- } \\
\text { ate } \\
\text { to } \\
\text { high }\end{array}$ & $\begin{array}{l}\text { Moder- } \\
\text { ate }\end{array}$ & 27 & 20 & 11 & 15 & -0.02 & Low & $\begin{array}{l}\text { Moder- } \\
\text { ate }\end{array}$ & & & & & & & \\
\hline $\begin{array}{l}\text { Paleocene } \\
\text { to Upper } \\
\text { Cretaceous }\end{array}$ & 24 & 24 & 8 & 13 & -0.11 & $\begin{array}{l}\text { Low } \\
\text { to } \\
\text { moder- } \\
\text { ate }\end{array}$ & $\begin{array}{l}\text { High } \\
\text { to } \\
\text { low }\end{array}$ & 28 & 19 & 19 & 9 & 0.26 & High & $\begin{array}{c}\text { High } \\
\text { to } \\
\text { low }\end{array}$ & & & & & & & \\
\hline
\end{tabular}

Note: $\mathrm{Md}_{1}=$ average median of the total silt fraction: $\mathrm{Md}_{2}=$ average median of the decalcified silt fraction: $\mathrm{V}_{1}, \mathrm{~V}_{2}=$ variation coefficient of total and decalcified silt: median $\left(V=\frac{S x}{x} \times 100\right) r=$ coefficient of correlation.

the dissolution rates were moderate. This accounts for the low sedimentation rates characteristic of the interval.

\section{Sub-Unit 2A (Cores 41 to 37)}

This unit comprises dominantly lower Miocene marly chalk interbedded with nannofossil chalk, calcareous claystone, siliceous nannofossil chalk and siliceous mudstone; these sediments accumulated at the rate of 1 $\mathrm{cm} / 1000 \mathrm{yr}$. Intense bioturbation prevails throughout the sub-unit. Sponge spicules constitute 5 to 20 per cent of the total sediment and reach up to 40 per cent in laminated, few to 5 -cm-thick lenses.

In the coarse fraction siliceous components range between 10 and 48 per cent and consist mostly of sponge spicules $(15-46 \%)$ and radiolarians $(0-16 \%)$. The carbonate component varies highly $(9-91 \%)$ and consists of benthic and planktonic foraminifers and unspecified debris. The abundance of the benthic foraminifers and unspecified debris, the relative scarcity of the planktonic foraminifers, the presence of abundant broken tests, and the evolution of the $\mathrm{PF} / \mathrm{BF}$ ratio suggest that active dissolution prevailed. This is confirmed by the frequency of fish debris that reaches up to 6 per cent. The terrigenous component is relatively low. Quartz averages about 2 per cent, muddy aggregates of undefined nature range from 0 to 33 per cent.
Median values of the silt are relatively high ( 25 and 23 $\mu \mathrm{m})$, thus suggesting that a relatively active bottom current regime continued to prevail, but the correlation coefficient is negative $(-0.31)$, thus suggesting that factors other than hydrodynamic (dissolution?) control the texture of the bulk silt fraction.

These observations indicate that Sub-unit 2A sediments were deposited under a relatively active bottom current regime and high dissolution rate. The 2-m.y. hiatus may be explained to have resulted from one or both influences.

\section{Unit 1}

\section{Sub-Unit 1B (Hole 400A, Cores 36 to 7)}

Middle Miocene to upper Pliocene light bluish gray to bluish white nannofossil chalk and ooze comprise most of Sub-unit 1B. Intercalated through most of the section are greenish gray marly nannofossil ooze and chalk beds, on the order of 20 to $30 \mathrm{~cm}$ thick; they occur at intervals ranging from 1 to 3 meters (Plate 3). There is an upward decrease of the $\mathrm{CaCO}_{3}$ content with maximum concentration in the middle Miocene. Siliceous sponge spicules occur sporadically in the section. Moderate to intense bioturbation occurs throughout; trace fossils identified through much of the section include Zoophycos, Teichichnus, halo, composite and rind burrows, that are often characterized by 


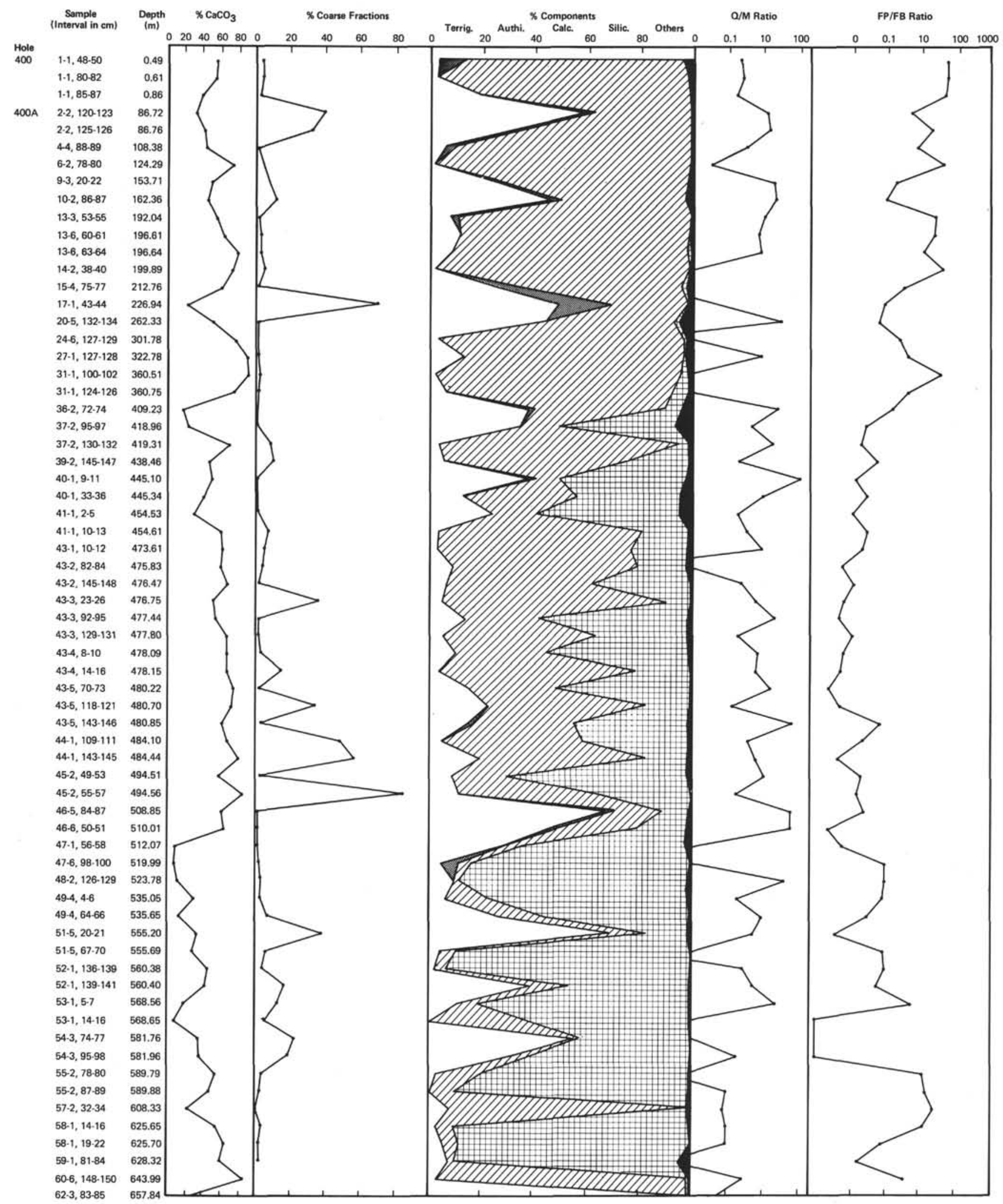

Figure 6. Coarse fraction, Site 400. 


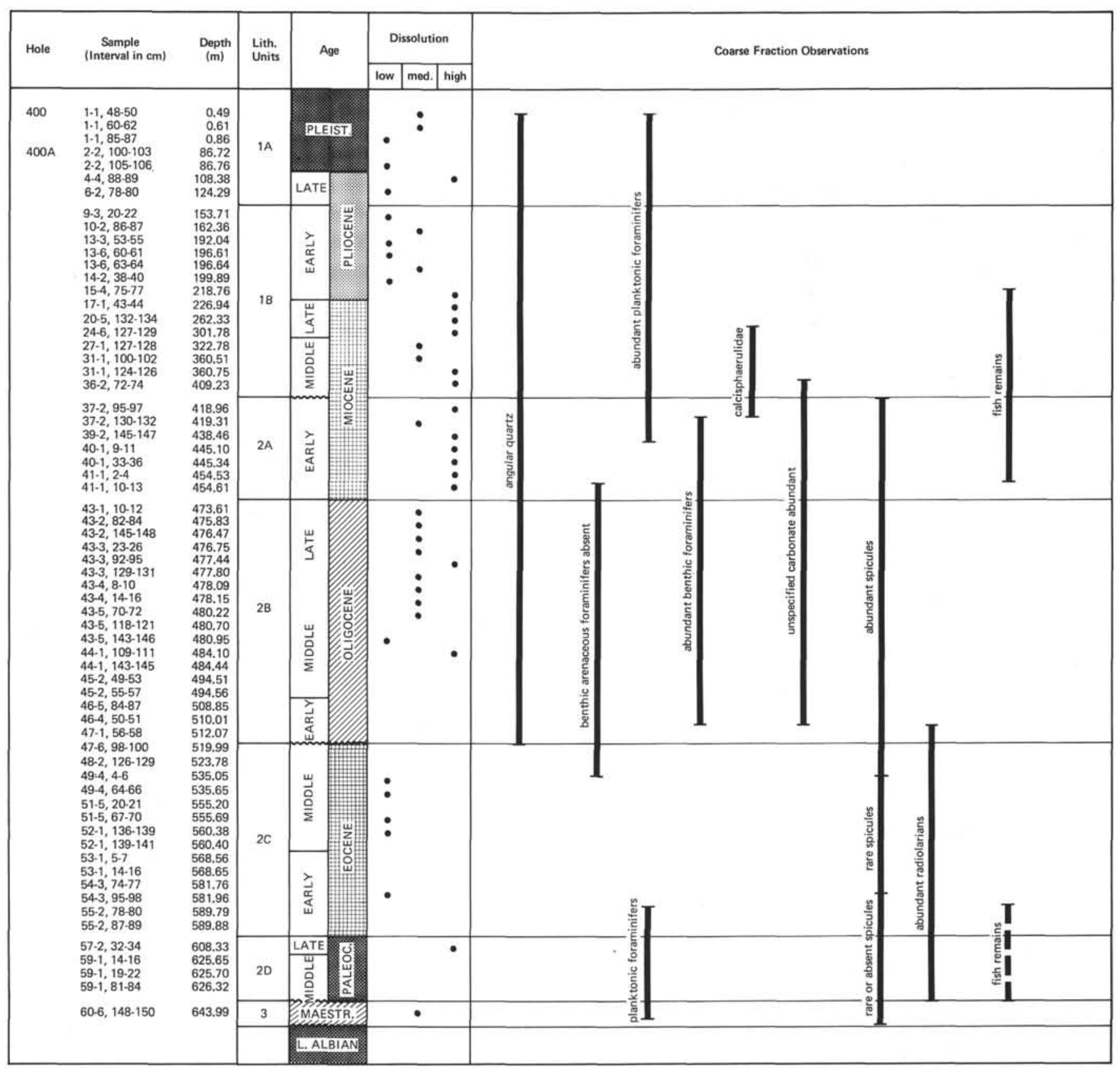

Figure 7. Evolution of coarse fraction, Site 400.

pyrite concentrations, and abundant fecal pellets. The sequence accumulated at rates ranging from $2.2 \mathrm{~cm} / 1000 \mathrm{yr}$ in middle-late Miocene to $5 \mathrm{~cm} / 1000 \mathrm{yr}$ during the Pliocene.

The carbonate content of the coarse fraction provides evidence of high dissolution rates during middle and late Miocene (low PF-BF ratio). Noteworthy is the occurrence of abundant algal cysts (Rögl and Hochuli, 1976) in Cores 20 to 36 .

Textural parameters of the silt fractions $(20 \mu \mathrm{m})$ indicate that a predominantly weak bottom regime prevailed during middle-late Miocene. Increasing median values (24 and 21 $\mu \mathrm{m})$ and a positive correlation coefficient $(0.65)$ indicate a marked increase of the bottom current regime in the early Pliocene.
Predominantly weak bottom current conditions prevailed during middle-late Miocene, and low PF-BF ratios indicate a high dissolution rate. In contrast, lower Pliocene sediments were deposited under a predominantly active bottom current regime while carbonate dissolution decreased. The sedimentation rate increase that occurred at the Miocene/Pliocene boundary may have been favored by lowered dissolution and increasing terrigenous supply.

\section{Sub-Unit 1A (Hole 400A, Cores 6 to 1)}

This sub-unit consists of alternating upper Pliocene to Quaternary nannofossil ooze and marly ooze deposited at the rate of $5.4 \mathrm{~cm} / 1000 \mathrm{yr}$. It is distinguished from Sub-unit IB on the basis of lower $\mathrm{CaCO}_{3}$ content. The nannofossil 
ooze occurs in beds on the order of 0.5 to 1 meter thick, separated by 25 to $50 \mathrm{~cm}$ thick (or less) beds of marly ooze. In many cases the marly zones are color banded at a still finer scale $(5-10 \mathrm{~cm})$ (Plate 3$)$. These changes correspond approximately to periods of time on the order to 20,000 years that may be related to climatic cyclicity.

The uppermost sediments recovered at Site 400 consist of alternating late Quaternary nannofossil ooze and diatomaceous nannofossil ooze. Samples 4-4, 88-90 cm and $6-2,78-80 \mathrm{~cm}$ are, respectively, late Pliocene homogeneous light olive-gray (5 Y 6/1) marly nannofossil ooze and light gray (N 7) nannofossil ooze. The textural parameters (21-19 and $22-18 \mu \mathrm{m})$, respectively, suggest low bottom current conditions. Samples 400A-2-2, 122-123 cm and 2-2, $125-126 \mathrm{~cm}$ belong to an interval of coarse silt; they are marly ooze, the coarse fractions ( 35 to $40 \%$ ) of which comprise angular quartz (28 to $52 \%)$ and planktonic foraminifers ( 25 to $59 \%$ ). The high proportion of these two components suggests erosion (or non-deposition) of the finer size grades. The median of the silt fraction $(25-25 \mu \mathrm{m}$ or 24-20 $\mu \mathrm{m}$ ) also suggests a relatively active current regime. These levels may be interpreted as contourite-type deposits. Sample 1-1, 48-50 cm is a dusky yellow (5 Y 6/4) marly foraminifer nannofossil ooze that constitutes the base of a $20-\mathrm{cm}$-thick sequence of greenish gray to grayish orange nannofossil ooze. According to the shipboard report, it could comprise as much as 10 per cent organic carbon; diatoms account for up to 5 per cent and the coarse fraction consists of 80 per cent planktonic foraminifers, 2 per cent radiolarian, and 10 per cent pyrite. According to its textural parameters, this sediment was deposited under relatively mild currents. The high terrigenous, diatom, and organic matter content indicates that it may have resulted from a high productivity episode. Samples 400-1-1, 60-62 cm and 400-1-1, 85-87 cm are marly ooze deposited also under low bottom current conditions.

In summary, the sediments of Core 1 were deposited during a period of weak bottom current activity, reduced dissolution, and occasional high productivity rates.

\section{Discussion (Figure 2; Table 6)}

A pelagic sedimentary regime, characterized by low dissolution rates, prevailed during the late Maestrichtian, thus the 10-m.y. early Paleocene hiatus does not appear to be a consequence of a high dissolution episode. In the Paleocene, bottom current activity remained low to moderate, while the dissolution rate increased. During lower and middle Eocene, an enhancement of the bottom current intensity occurred, while dissolution decreased. The increase of the sedimentation rate from $0.5 \mathrm{~cm} / 1000 \mathrm{yr}$ in late Paleocene to $1.3 \mathrm{~cm} / 1000 \mathrm{yr}$ in early-Eocene is a consequence of decreasing dissolution rates and increasing terrigenous supply and productivity. The late Eocene hiatus appears to have been the consequence of erosion by bottom currents. The abrupt lithological change that occurs at the base of the lower Oligocene probably reflects a major event of the evolution of the northeast Atlantic. An active (though highly fluctuating) bottom current regime and moderate dissolution rate prevailed during this time. Evidence for the occurrence of turbidity currents at that time is not obvious, but the possibility cannot be ruled out in the present state of study.

During early Miocene, bottom current intensities were predominantly high, and interbedded claystones point to the occurrence of episodic rise of the CCD. The intra-lower Miocene may have resulted as a consequence of the conjunction of these two influences. Bottom current intensities decreased during middle-late Miocene, while high dissolution prevailed. The increase of the sedimentation rate from $1 \mathrm{~cm} / 1000 \mathrm{yr}$ in the early Miocene to 2.2 $\mathrm{cm} / 1000 \mathrm{yr}$ in middle Miocene, in spite of the high dissolution rates, implies increasing productivity and terrigenous supply. At the onset of the early Pliocene bottom current intensity increased notably, but decreasing dissolution and increasing terrigenous supply lead to a marked increase of the sedimentation rate $(2.2$ to $5.3 \mathrm{~cm} / 1000 \mathrm{yr})$.

\section{STRATIGRAPHIC EVOLUTION}

\section{Upper Cretaceous}

The lowermost Upper Cretaceous hiatus that occurs at the three sites is a prominent feature of the margin stratigraphy. At Sites 401 and 402, it happened during the transition from a neritic (Pastouret et al., 1974; Pastouret and Auffret, 1976) to a pelagic sedimentary regime, in a period of rising sea level (Cenomanian transgression) and low terrigenous supply from a continental area of low relief. At Site 400, the origin of the hiatus is questionable; possibly slope instability was one of the involved factors. Hiatuses in the lowermost Upper Cretaceous are common in the North Atlantic (Hollister, Ewing, et al., 1972; Rona, 1973; Auffret and Pastouret, in press). The occurrence of this hiatus in varied physiographic situations and its wide extension suggest that it may signify a large-scale oceanic event. It is well known that major changes affected the sea-floor-spreading regime in the North Atlantic during the Albian, including onset of sea-floor spreading in the Bay of Biscay (Williams, 1975; Olivet et al., 1976a). These could have affected the hydrologic and physiographic realm and led to non-deposition. The modalities of such an alteration of the sedimentary regime are still poorly understood.

At Site 401 , the Campanian and Maestrichtian grayish orange laminated nannofossil chalks accumulated at a very slow rate $(0.2 \mathrm{~cm} / 1000 \mathrm{yr})$; dissolution rates were low, while active bottom current dominantly prevailed. At Site 400, reddish yellow marly nannofossil chalk and white to grayish orange marly calcareous chalk of late Maestrichtian age occur above the hiatus. Low dissolution rates and low bottom current activity prevailed at this site during this time. At Site 402, no Upper Cretaceous sediment has been recovered. The reduced thickness of the recovered Upper Cretaceous sections is also confirmed by the rarity of Upper Cretaceous rocks dredged from the margin. Where they do occur, they consist of condensated section, such as the phosphatic crust superimposed on granite outcrops at about 3000 meters depth on the Goban Spur (Pautot et al., 1976; Auffret et al., this volume).

The much reduced thickness of the uppermost Cretaceous sections from continental margins often has been reported (Rona, 1973; Worsley, 1974). The latter author suggests that 
a global climate deterioration may have been responsible for the sudden extinction of many biologic groups. Colder surface water $\left(14^{\circ} \mathrm{C}\right)$ during this time has been reported by Létolle et al. (this volume). Also noteworthy is the fact that accumulation of sediment during Campanian and Maestrichtian was contemporaneous with the beginning of the regression; this in turn may have favored an increase of terrigenous and biogenic supplies.

\section{Paleocene}

At Sites 401 and 400 , increasing surface water productivity characterized the margin environment at the onset of the Cenozoic, but the sediments at Site 400 appear to have been submitted to more active dissolution and to a less active bottom current regime than those at Site 401. This suggests that the foraminifer lysocline level was at a water depth somewhere between those of the two sites (a situation not so much different from today's; Auffret and Pastouret, 1977). Nevertheless, the average late Paleocene carbonate sedimentation rates (Figure 2) were almost identical at Sites 400 and 401 . Important paleogeographic events that affected the northeast Atlantic occurred during the Paleocene (Greenland-Iceland separation, Montadert et al., 1977). Increasing terrigenous supplies, and the occurrence of attapulgite and sepiolite suggest a warm and humid climate; warm surface waters are also reported by Létolle et al. (this volume). These observations suggest that, in the uppermost Cretaceous and Paleocene time, formation of cold bottom water was initiated in the northern area, while circulation of warm surface water from a southern origin was activated, thus starting the process of commotion in the ocean (Berggren and Hollister, 1977).

\section{Eocene}

Lower and middle Eocene sediments were recovered at Site 400. At Site 401, lower, middle, and the uppermost Eocene are present; in contrast, at Site 402, upper Eocene only was recovered, superimposed on Albian shallow water limestone. Thus, the upper Eocene hiatus appears to have had its maximum development in the deeper parts of the margin.

Figure 2 shows the parallel evolution of the carbonate, silica, clay, and quartz sedimentation rates at Sites 400 and 401 during early and middle Eocene time. Silica sedimentation increased markedly at both sites during this period. During late Eocene time, carbonate sedimentation rates were slightly higher at Site 402 than at Site 401 , whereas the silica accumulation rates were about four times greater in the former, thus pointing to the possible influence of a near-shore area.

The sedimentary regime during early middle Eocene was characterized by a more active bottom current regime at Site 400 than at Site 401 , a situation opposite to that during the Paleocene. During middle Eocene dissolution decreased slightly which favored increase of the sedimentation rate observed at Site 401, supplemented also by increasing terrigenous and biogenic siliceous supplies. The increase in terrigenous supply could in turn be related to tectonic activity in the Pyrenean.

The strengthening of the bottom water circulation during the Eocene has been attributed by Berggren and Hollister (1977) to the onset of the overflow of the Norwegian Sea bottom water above the Iceland-Faeroe Ridge. However, according to the result of Leg 38 (Talwani, Udintsev, et al., 1976) this ridge subsided substantially below sea level only in middle Miocene time. Laughton, Berggren, et al. (1972) interpreted the upper Eocene hiatus to be a result of the Pyrenean orogeny but, as in the lowermost Upper Cretaceous, it may have a more general significance. Effectively, Olivet et al. (1976b) pointed to the occurrence of a middle to upper Eocene acoustic unconformity in a large area off Iberia and in the Bay of Biscay. Moreover, according to Sheridan (1976) a break in the sedimentary regime occurred also on the western margin of North Atlantic as a consequence of a change in sea-floor-spreading regime in the North Atlantic. Hence it seems highly probable that the late Eocene hiatus in the Bay of Biscay is related to an event of oceanic rather than regional scale.

\section{Oligocene}

Important changes occur in the Oligocene section, particularly at Site 400 where greenish gray siliceous marly nannofossil chalk overlies the dominantly orange pink Eocene chalk. In addition, there is a marked change in the silica component wherein sponge spicules became predominant instead of radiolarians, and the dissolution rate notably increased. At Site 400 , episodes of high bottom current intensity probably were responsible for the deposition of the coarse layers that consist mostly of sponge spicules. Dissolution at this site was moderate; at Site 401 it seems to have been mainly low during the same period. The high bottom current intensity and moderate dissolution rate resulted in the low sedimentation rate $(0.35 \mathrm{~cm} / 1000 \mathrm{yr})$ observed at Site 400 . This evolution is concomitant with a severe cooling of surface and bottom water masses $\left(11^{\circ}\right.$ and $7.5^{\circ} \mathrm{C}$, respectively, Letolle, this volume). This is the time of the settling of the psychrosphere (Benson, 1975; Ducasse and Peypouquet, this volume); a geochemical break is also observed at this level (Debrabant et al., this volume). This global cooling of the hydrosphere is contemporaneous with the onset of the formation of sea ice in the Southern Hemisphere 38 m.y. ago, near the Eocene/Oligocene boundary, and of a general drop of the CCD in the equatorial zones (Kennett, 1977). It is very probable that the abrupt change in the lithology during the early Oligocene in the Bay of Biscay is a consequence of a northward extension of the influence of the Antarctic Bottom Water Current.

\section{Miocene}

Lower Miocene is present at Site 400. It comprises interbedded nannofossil chalk, claystone, siliceous marly chalk, and siliceous mudstone. The accumulation rate increased notably compared to the Oligocene $(1.0 \mathrm{~cm} / 1000$ yr). High dissolution rates are deduced from observation of the coarse fraction; bottom currents, although fluctuating, were still active. The lower Miocene regime thus appears to have been a continuation of that of the Oligocene, with the exception of the enhancement of calcium carbonate dissolution. The intra-lower Miocene hiatus hence may be related to high dissolution rates and/or strengthening of the bottom currents. Tectonic instability at the Oligocene/Miocene boundary has been reported from the Aquitaine Basin (Dupouy-Camet, 1952), but at the 
lower-middle Miocene boundary (14 m.y. Langhian) the southern margin of the European plate was affected by an important tectonic phase; Olivet et al., 1976b) attribute to this event an acoustic reflector unconformity in the Bay of Biscay. A hiatus also has been observed in early Miocene at Site 118 (Laughton, Berggren, et al., 1972).

The high terrigenous supply and productivity rates that are characteristic of Miocene are probably a consequence of high erosion rates on the uplifted continental area; middle and late Miocene are characterized by a further increase in the accumulation rates $(2.2 \mathrm{~cm} / 1000 \mathrm{yr})$. In middle Miocene, contribution of the silica component to the accumulation rate decreased slightly. This was a time of predominantly pelagic carbonate regime, weak bottom currents and moderate to high dissolution rates, which agrees well with the CCD rise proposed by several authors (Heath, 1969; Berger, 1974; Berger and Winterer, 1974; van Andel et al., 1975). Furthermore, this high dissolution episode occurred at a time of predominantly low bottom current regime and possibly low water mass turnover. During the late Miocene, the terrigenous supply distinctly increased, possibly as a consequence of the worldwide regression related to the development of the Antarctic ice sheet (Kennett, 1977). Isolation of the Mediterranean basin is probably therein linked (Berggren and Hollister, 1977). Increased sedimentation on the upper slope, reported by Peypouquet (1977) in the Aquitaine basin, indicate a prograding margin (and a tectonic uplift ?) resulting in the transition from epibathyal $(600-800 \mathrm{~m})$ to a littoral environment during the course of the Miocene.

\section{Pliocene}

At Site 400 the lower Pliocene section consists of nannofossil ooze and marly nannofossil ooze that were deposited under the control of active bottom currents and decreasing dissolution. These two observations are consistent with an increasing turnover rate of the water masses. As a consequence of higher terrigenous supply and of lesser dissolution the sedimentation rate increased from 1 to $5.3 \mathrm{~cm} / 1000 \mathrm{yr}$. The upper Pliocene nannofossil ooze and marly nannofossil ooze are interbedded at approximately 1 meter. These rhythmic changes represent an approximate duration of 20,000 years. The alternation may be a consequence of climate oscillation, from cold dry periods (nannofossil ooze) to temperate and humid (marly nannofossil ooze) times. The appearance of this cyclic change during the late Pliocene corresponds with the onset of the glaciation in the Northern Hemisphere, 2.5 to 3 m.y. ago (Berggren and Hollister, 1977; Kennett, 1977).

\section{Quaternary}

As a consequence of poor core recovery, and of the varying conditions that are characteristic of this period, the following remarks are made only as example of some of the sedimentary regime that prevailed on the margin during Quaternary time.

Calcareous mud and marly ooze were sampled at Site 402. An active bottom current regime appears to have been prevailing during their deposition. It appears that the sedimentary structures observed (graded beds, coarse laminae) could be the result of transport by oceanic bottom currents, the role of turbidity currents being subordinate. Marly calcareous ooze, calcareous ooze, and calcareous mud have been also recovered at Site 401; they appear also to have been deposited under a relatively active bottom current regime and do not imply turbidity current activity.

Interbedded Quaternary nannofossil ooze, marly ooze, and calcareous mud sampled at Site 400 appears to have been controlled by bottom current of fluctuating intensity. No evidence of turbidity current activity has been observed.

It thus appears that the role of turbidity currents was subordinate at the three sites. Although further study is to confirm this point, it is clear that their physiographic location may explain such a paucity. Sites 402 and 401 are both on a sedimentary apron, and Site 400 seems to have been protected by a topographic barrier from turbidity currents flowing down the Blackmud canyon.

\section{Summary}

The sedimentary evolution of the Armorican margin is characterized by two cycles wherein a period characterized by low sedimentation rates (Upper Cretaceous to lower Paleocene, and Oligocene) was followed by a period of increasing sedimentation rates (upper Paleocene-middle Eocene, Miocene to Quaternary). The duration of the period of reduced accumulation rates or hiatuses are, respectively, about $40 \mathrm{~m} . \mathrm{y}$. and $22 \mathrm{~m} . \mathrm{y}$. The two periods of active deposition were, respectively, about 10 and $22 \mathrm{~m} . \mathrm{y}$. long. The durations of the two cycles are, respectively, 50 and 44 m.y. The first one may be related to the thermal subsidence of the margin (Sleep, 1971) and is in good agreement with the onset of spreading in the Bay of Biscay in Albian time.

The second phase of reduced accumulation rate (or hiatus) in the late Eocene-Oligocene is apparently related to a strengthening of the bottom water circulation that may be related to the initiation of the flow of the Antarctic Bottom Current. Changing paleogeography during the Miocene in relation to Alpine orogeny and the onset of the overflow regime over the Iceland-Scotland Ridge resulted in a new episode of high sedimentation rates.

\section{CONCLUSION}

In this chapter, we have used our analytical results (lithology and textures) to reconstruct the sedimentary evolution at the three sites drilled on the Armorican margin. It appears to us that the textural properties of the sediment (and more particularly their silt fraction) may record the fluctuation of bottom currents, and is consequently of primary importance for reconstruction of past oceanographic conditions. It is evident, however, that it is only by the confrontation of all data, including sedimentation rates, sedimentary structures, bulk mineralogy, composition of the coarse fraction, etc., that one can decipher the signification of the recovered sediments. In the course of this study, numerous limiting factors have arisen, such as the statistical significance of the textural data, and the influence of the lithological composition on the textural parameters. Although the agreement of our conclusions with those derived from other methods is generally good, we conclude that study on a finer scale should be undertaken in order to avoid this bias. From this study, important aspects of the evolution of the sedimentary regime are: 
1) a low sedimentation rate in the uppermost Cretaceous related to a possible climate deterioration and high sea level;

2) settling of an active bottom current circulation during the early Eocene at Site 400, concomitant with a clear thermal stratification of the water masses;

3) highly fluctuating bottom current regime during the Oligocene, spicule-rich "contourites", being emplaced during periods of active bottom current;

4) important changes in the sedimentary regime at the early-middle Miocene boundary characterized by decreasing bottom currents and an abrupt increase in the sedimentation rates (in spite of high carbonate dissolution);

5) active bottom current, decreasing dissolution and marked increase of the sedimentation rate in the early Pliocene.

The lowermost Upper Cretaceous hiatus and the upper Eocene hiatus are probably the result of the hydrologic and physiographic environment on an oceanic scale.

\section{ACKNOWLEDGMENTS}

We thank those scientists and technicians who have been working at sea or in shore-based laboratories, on DSDP-IPOD Leg 48 material, and particularly Hervé Chamley and Jörn Thiede for their critical reading of a first draft of this work. The compilation of a great number of lithological data has been possible through assistance provided by D. Courtay and A. Rue from S.E.O. (COB). Suzanne Marques and René Kerbrat have been in charge of the technician procedure and analyses. To all people who collaborated in this work we express our grateful acknowledgment for their kind and efficient support.

\section{REFERENCES}

Auffret, G.A. and Pastouret, L., 1977. Sur les modalités de la sédimentation profonde actuelle dans le golfe de Gascogne, Bull. Soc. Géol. France, v. 19, p. 243-248.

, in press. Evolution sédimentaire comparée des marges armoricaine et de Blake-Bahama depuis le Crétacé supérieur, d'après les résultats des Legs 48 and 44 du Glomar Challenger, Bull. Soc. Géol. France.

Auffret, G.A., Pastouret, L., and Kerbrat, R., 1975. Dynamique sédimentaire au bas de la marge continentale armoricaine; exemple de la ride Aegis, Actes IX, Congrès de Séd., Nice, thème 6 .

Benson, W.H., 1975. The origin of the Psychrosphere as recorded in changes of deep-sea ostracodes assemblages, Lethaia, v. 8 , p. 69-83.

Berger, W.H., 1974. Deep-sea sedimentation. In Burk, C.A. and Drake, C.L. (Eds.), The geology of the continental margin: Berlin, Heidelberg, New York (Springer-Verlag), p. 213-241.

Berger, W.H. and Winterer, E.L., 1974. Plate stratigraphy and the fluctuating carbonate line, Spec. Publ.Int. Assoc. Sedimentol., v. 1, p. 11 .

Berggren, W.A. and Hollister, C.D., 1977. Plate tectonics and paleocirculation-commotion in the ocean, Tectonophysics, v. 38 , p. 11-48.

Berthois, L. and Auffret, G.A., 1965. Application d'un compteur électronique à la mesure des vitesses de chute des particules fines, C.R. Acad. Sci. Paris, v.261, p. 5165-5167.

Dupouy-Camet, J., 1952. Recherches structurales sur les accidents triasiques du sud-ouest de l'Aquitaine, Bull. Carte Géol. Fr., no. 233 .

Elwood, B.B. and Ledbetter, M.T., 1977. Antarctic bottom water fluctuations in the Vema Channel: effects of velocity changes in particle alignment and size, Earth Planet. Sci. Lett., v.35, p. 189.

Güssov, V., 1976. Sequence concepts in petroleum engineering, Geotimes, v. 21, p. 16-17.

Heath, G.R., 1969. Carbonate sedimentation in the abyssal equatorial Pacific during the past 50 million years, Geol. Soc. Am. Bull., v. 80 , p. 689.

Herman, Y., 1972. Origin of deep-sea cherts in the North Atlantic, Nature, v. 238 , p. 392-393

Hollister, C.D., Ewing, J.I. et al., 1972. Initial Reports of the Deep Sea Drilling Project, v.11: Washington (U.S. Government Printing Office).

Hollister, C.D. and Heezen, B.C., 1972. Geologic effects of ocean bottom currents. In Gordon, A.L. (Ed.), Studies in physical oceanography. A tribute to G. Wüst: New York (Gordon and Breach), p. 37-66.

Kennett, J.P., 1977. Cenozoic evolution of antarctic glaciation, the circum-Antarctic ocean and their impact on global oceanography, J. Geophys. Res., v. 82, p. 3825.

Laughton, A.S., Berggren, W.A. et al., 1972. Initial Reports of the Deep Sea Drilling Project, v.12: Washington (U.S. Government Printing Office).

Lisitzin, A.P., 1972. Sedimentation in the world ocean, Soc. Econ. Pal. Min. Spec. Publ. no. 17, p.1-218.

Montadert, L., Roberts, D.G., Auffret, G.A., Bock, W., Dupeuble, P.A., Hailwood, E.A., Harrison, W., Kagami, H., Lunsden, D.N., Müller, C., Schnitker, D., Thompson, R.W., Thompson, T.L., and Timofeev, P.P., 1977. Rifting and subsidence on passive continental margins in the northeast Atlantic, Nature, v. 268, p.305-308.

Olivet, J.L., Pastouret, L., Auzende, J.M., and Auffret, G.A., 1976a. Armorican margin evolution in relation with Bay of Biscay genesis, Paper presented at Colloque I.U.S.G.S. Durham, April.

Olivet, J.L., Bonnin, J., Auzende, J.M., 1976b. Manifestation des phases de compression tertiaire dans l'Atlantique nord-est, Paper presented at fourth reunion Sci. Terre, Paris, April 1976.

Pastouret, L. and Auffret, G.A., 1976. Observations sur les microfaciès des roches sédimentaires prélevées sur la marge armoricaine, Rev. Inst. Français du Pétrole, v. 31, p. 401-425.

Pastouret, L., Masse, J.P., Philip, J., and Auffret, G.A., 1974. Sur la présence d'Aptien inférieur à faciès urgonien sur la marge continentale armoricaine, conséquences paléogéographiques, C.R. Acad. Sci., Paris, v.278, p. 2011-2014.

Pastouret, L., Auffret, G.A., and Chamley, H., 1978. Microfacies of some sediments from the northwestern Atlantic: paleooceanographic implications (Leg 44 DSDP). In Benson, W.E., Sheridan, R.E., et al., Initial Reports of the Deep Sea Drilling Project, v. 44: Washington (U.S. Government Printing Office), p. 477-502.

Pautot, G., Renard, V., Auffret, G.A., Pastouret, L. and de Charpal, O., 1976. A granite cliff deep in the NorthAtlantic, Nature, v.263, p. 669-672.

Peypouquet, J.P., 1977. Les Ostracodes et la connaissance del paléo-milieux profonds. Application au Cénozoïque de 1'Atlantique nord-oriental, Thèse de Doctoral d'Etat, Bordeaux.

Rivière, A., 1952. Expression analytique générale de la granulométrie des sédiments meubles. Indices caractéristiques et interprétation géologique: Notion de faciès granulométrique, Bull. Soc. Géol. France, v. 6, p. 155-167.

Rögl, F. and Hochuli, P., 1976. The occurrence of Bolboforma, a probable algal cyst, in the Antarctic Miocene of DSDP Leg 35. In Craddock, C., Hollister, C.D. et al., Initial Reports of the Deep Sea Drilling Project, v.35: Washington (U.S. Government Printing Office), p. 713-719. 
Rona, P.A., 1973. Worldwide unconformities in marine sediments related to eustatic changes of sea level, Nature Phys. Sci., v. 244 , p. $25-26$

Sheridan, R.E., 1976. Sedimentary basins of the Atlantic margin of North America, Tectonophysics, v. 36, p. 113-132.

Sleep, N.H., 1971. Thermal effects of the formation of Atlantic continental margin by continental break up, Geophys. J. Roy. Astron. Soc., v. 24, p. 325-350.

Talwani, M.K., Udintsev, G., et al., 1976. Initial Reports of the Deep Sea Drilling Project, v.38: Washington (U.S. Government Printing Office). van Andel, T.H., Heath, G.R., and Moore, T.C., Jr., 1975. Cenozoic history and paleo-oceanography of the central equatorial Pacific Ocean, Geol. Soc. Am. Mem. 143, p. 134.

Williams, C.A., 1975. Sea-floor spreading in the Bay of Biscay and its relationship to the North Atlantic, Earth Planet. Sci. Lett., v. 24, p. 440-456.

Worsley, T., 1974. The Cretaceous Tertiary boundary events in the ocean. In Hay, W.W. (Ed.), Studies in paleooceanography: Soc. Econ. Paleontol. Min. Spec. Publ. 20, p. 94-120. 
APPENDIX A

Site 402

HOLES 402-402A

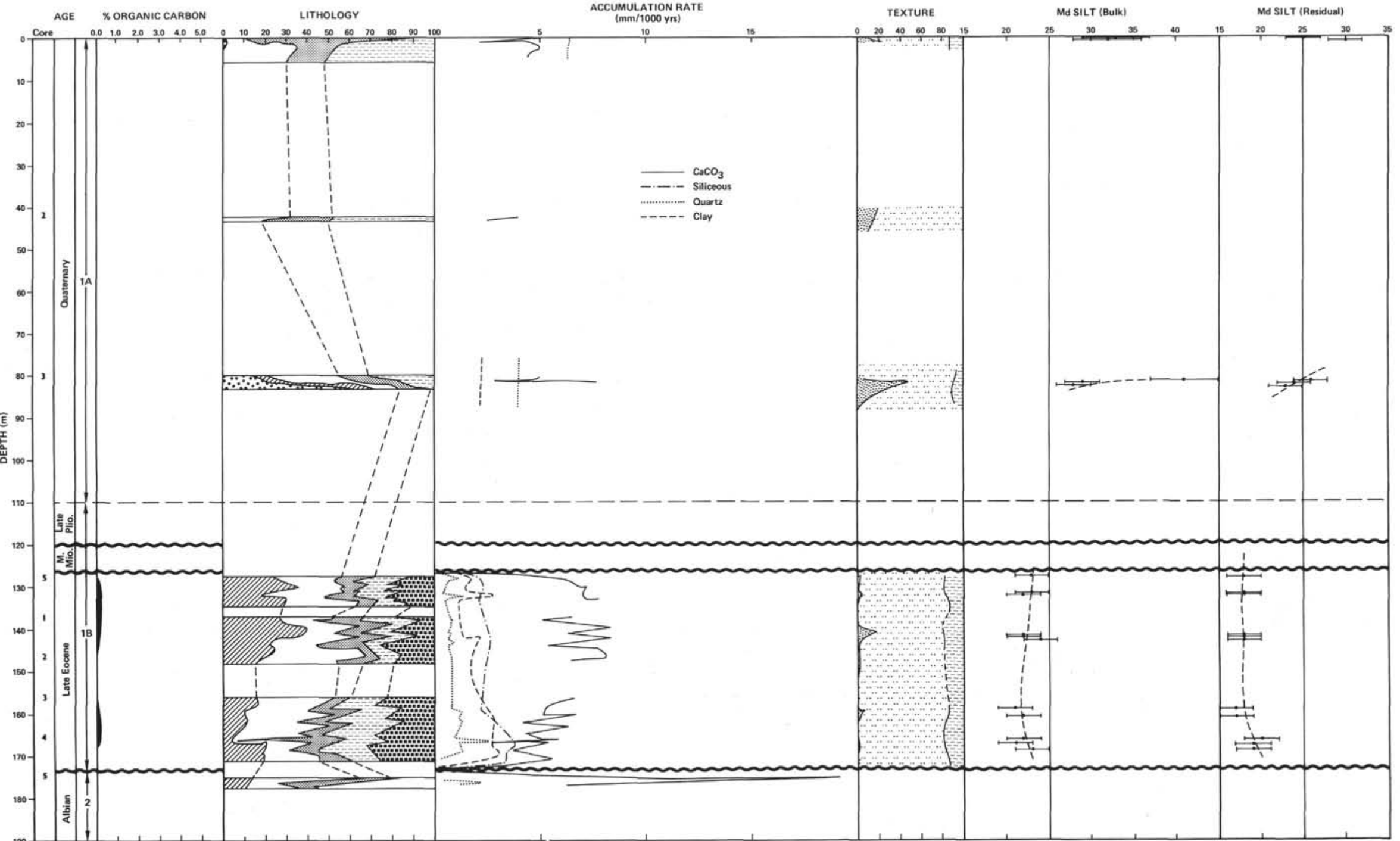

\begin{tabular}{|c|c|}
\hline Foraminiter & $\square$ Ouartz \\
\hline Nannotost & $\begin{array}{ll}\text { Clay and othe } \\
\text { fine material }\end{array}$ \\
\hline & \\
\hline
\end{tabular}




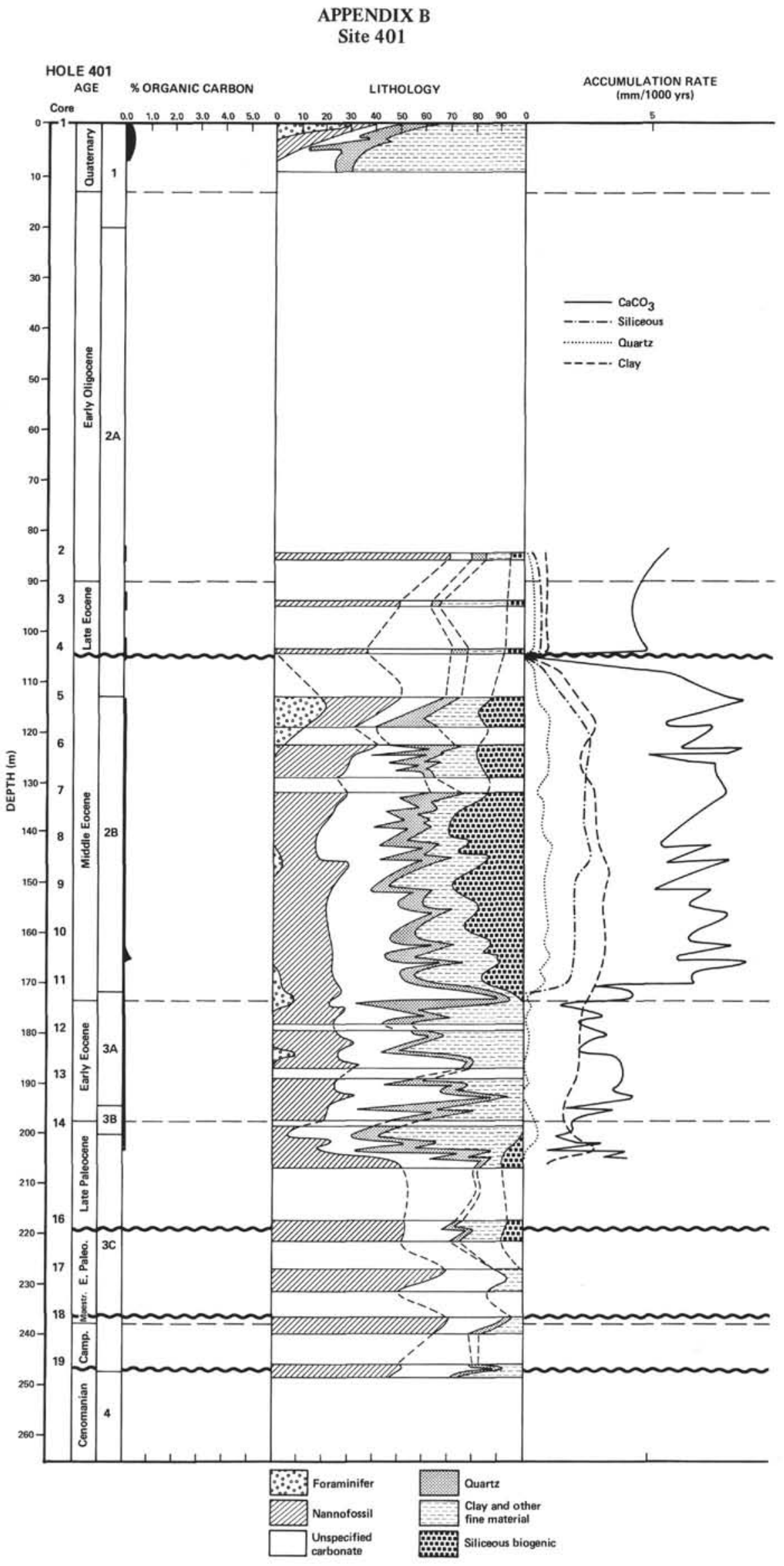




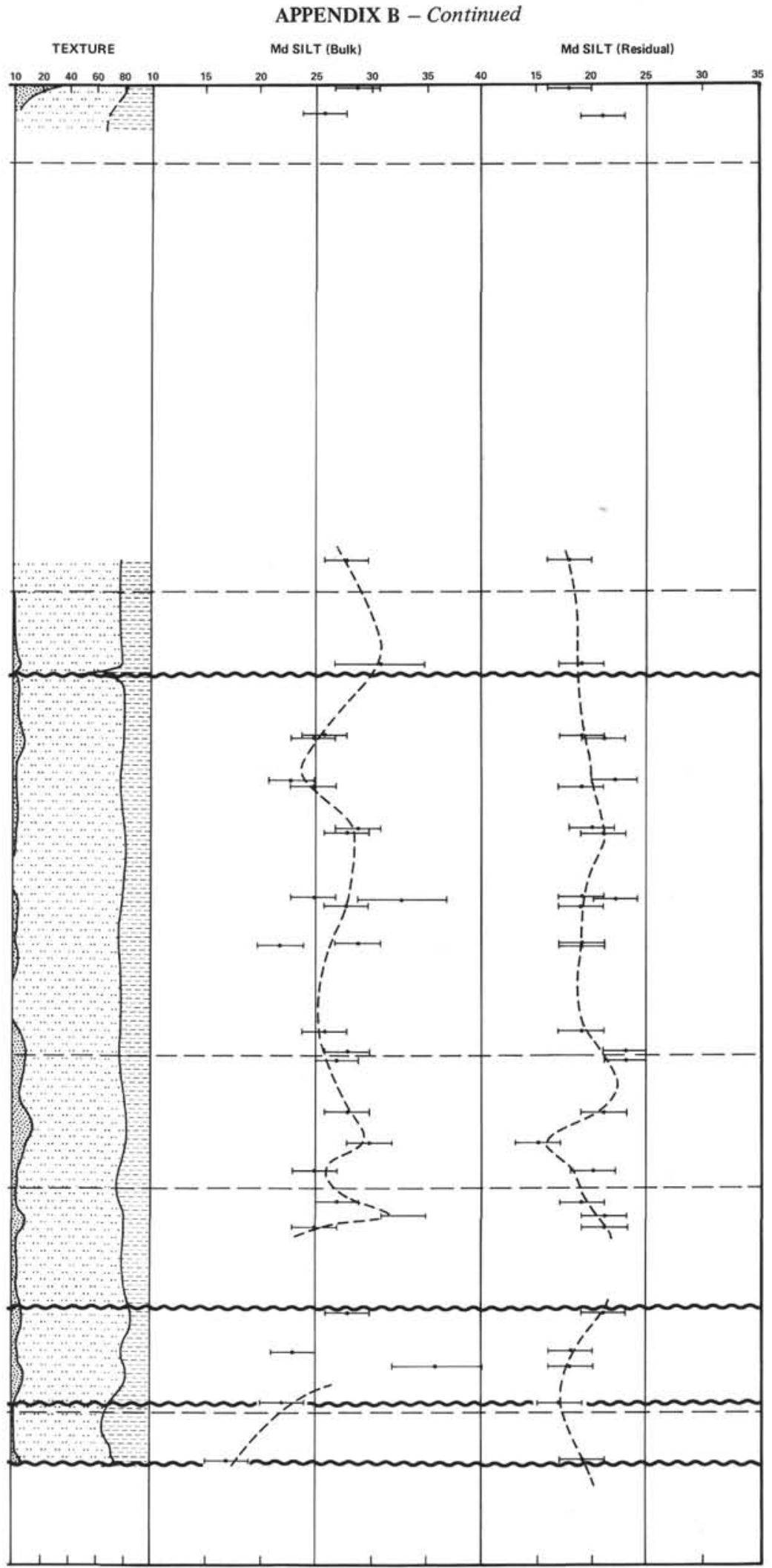


PLATE 1

Holes 402, 402A

Figure 1 Section 402-3-1. Textures and colors changes in Quaternary sediments (cf. Figure 4).

Figure 2 Section 402A-1-3. Debris flow (?) comprising limestone, mud gravels, echinoid spine, large foraminifers, glauconite, interbedded in late Eocene nannofossil chalk.

Figure 3 Section 402A-1-5. Slumped beds of late Eocene siliceous marly nannofossil chalk and interbedded cobble.

Figure 4 Section 402A-1-5. Middle Miocene chalk and lower Pliocene marly chalk recovered in a washed core. 
PLATE 1

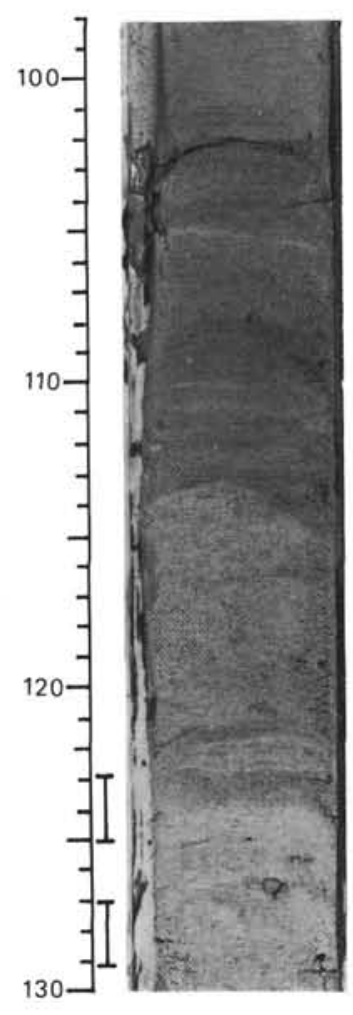

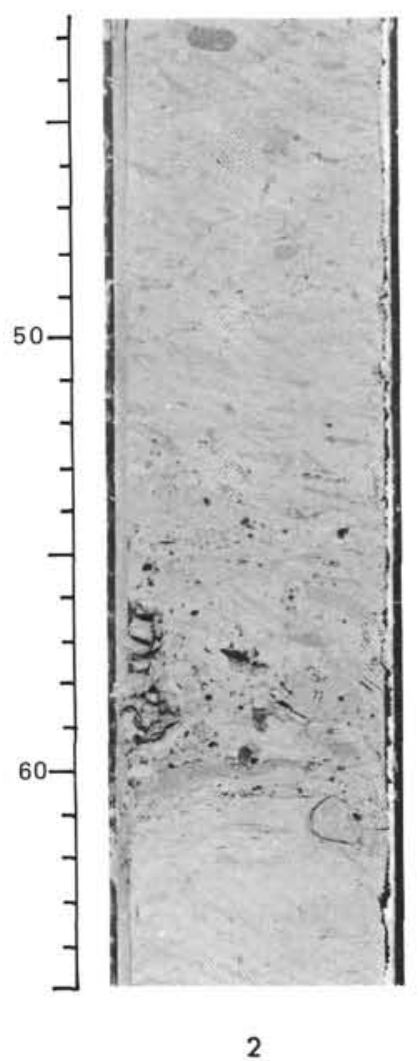
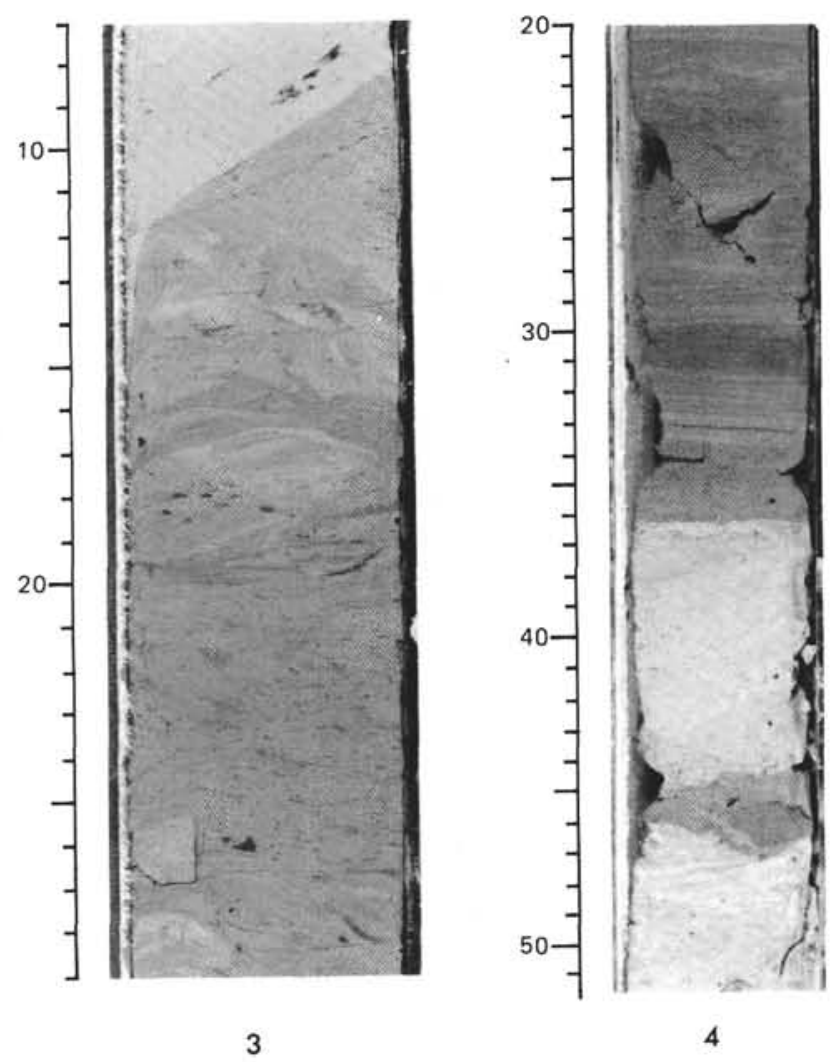
PLATE 2

Hole 401

Figure 1 Section 5-3. Interbedded middle Eocene nannofossil chalk and marly nannofossil chalk disturbed by mottling and compaction.

Figure 2 Section 13-4. Lower Eocene collophane bearing calcareous mudstone, interbedded in light brown nannofossil chalk.

Figure 3 Section 16-2. Large rind burrow in late Paleocene nannofossil chalk comprising 7 per cent sponge spicules. 
PLATE 2

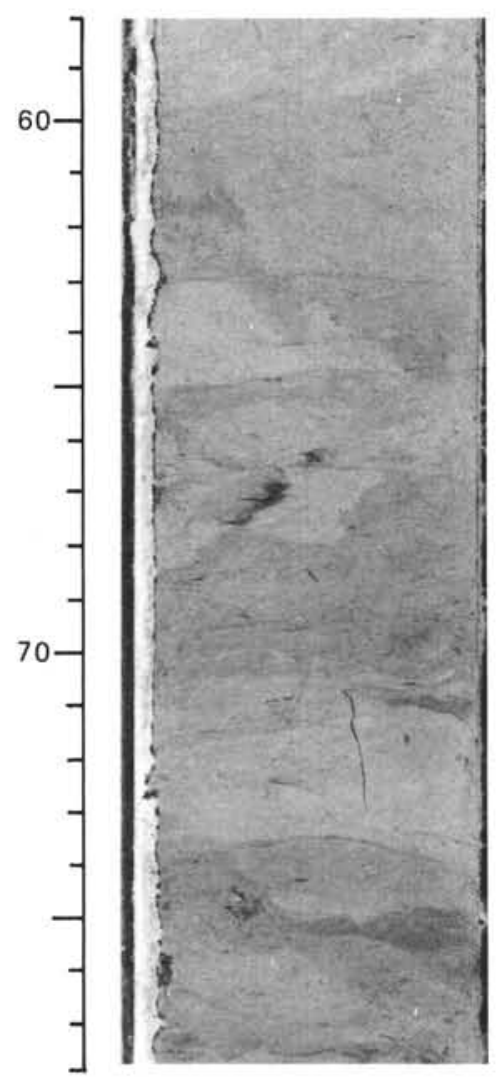

1

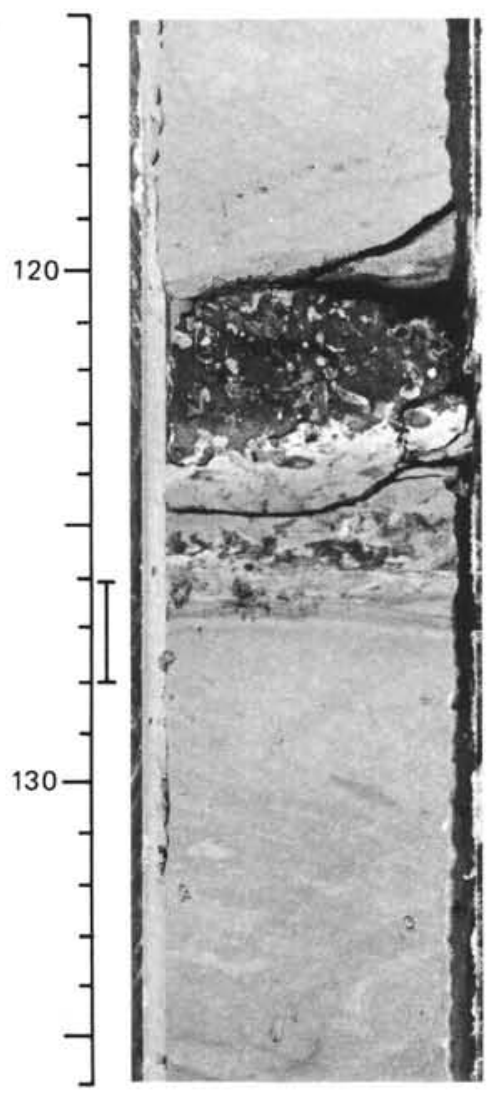

2

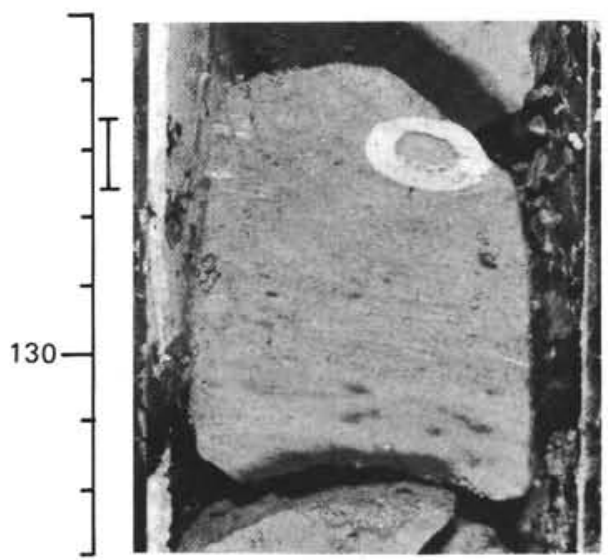

3 
PLATE 3

Hole 400A

Figure 1 Section 3-2. Interbedded darker Pleistocene marly nannofossil ooze and lighter bioturbated nannofossil ooze.

Figure 2 Section 17-3. Gradational transition between lighter late Miocene nannofossil chalk and darker marly nannofossil chalk with abundant Chrondite-type burrow.

Figure 3 Section 20-5. Fairly sharp contact between late Miocene marly nannofossil chalk and nannofossil chalk; observe the important burrowing at the boundary (Teichichnus-type burrow).

Figure 4 Section 36-2. Color and lithologic mottling due to bioturbation in transitional sediments at the boundary between Sub-units 1B and 2A. 
PLATE 3

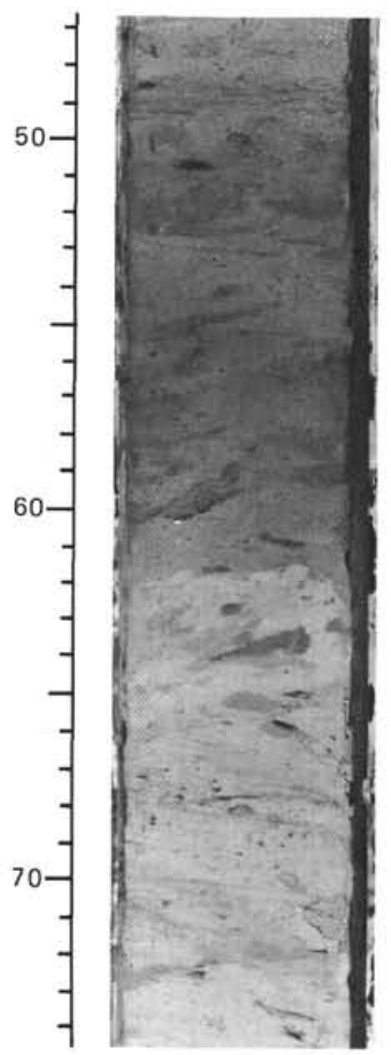

1

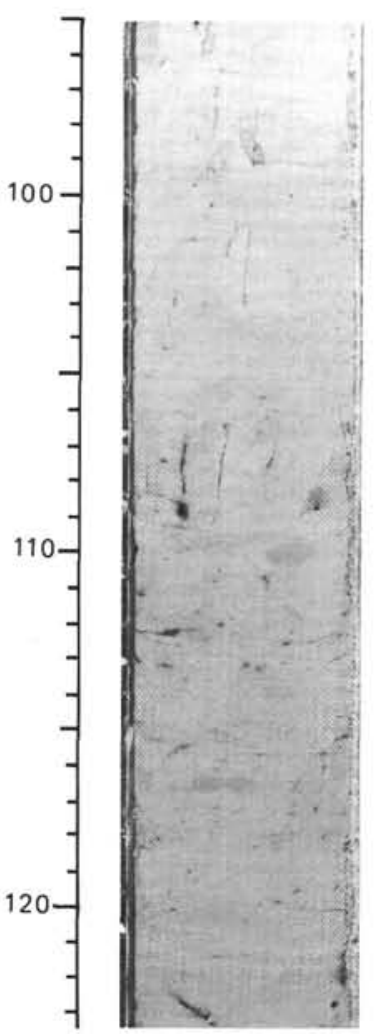

2

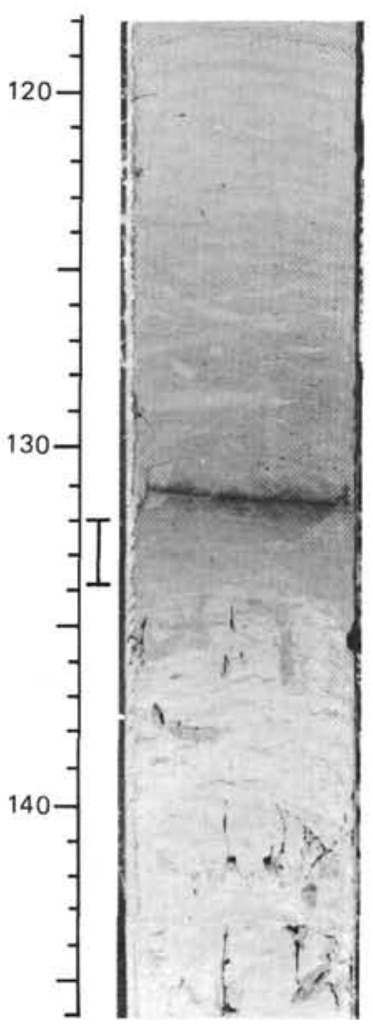

3

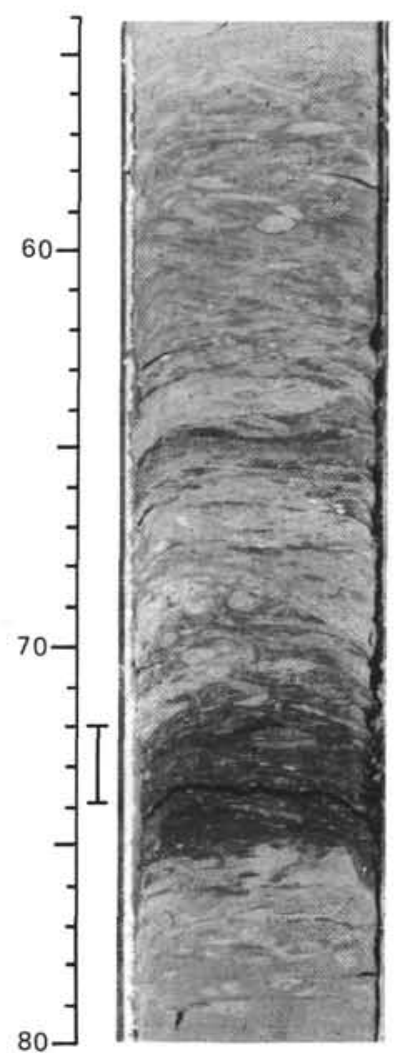

4 
PLATE 4

Hole 400A

Figure 1 Section 43-3. Spicule-rich silty layer of siliceous chalk in Oligocene marly nannofossil chalks section of Sub-unit 2B.

Figure 2 Section 43-5. Syndepositional slump in Sub-unit 2B marly nannofossil chalk; dark colored fragments contain Paleocene and Lower Cretaceous nannofossils.

Figure 3 Section 48-3. Subrounded Lower Cretaceous limestone cobble interbedded in middle Eocene siliceous mudstone.

Figure 4 Section 55-2. Interbedded early Eocene grayish orange and greenish gray to light greenish gray marly chalk. Location of studied sample has been indicated by a vertical line. 
PLATE 4

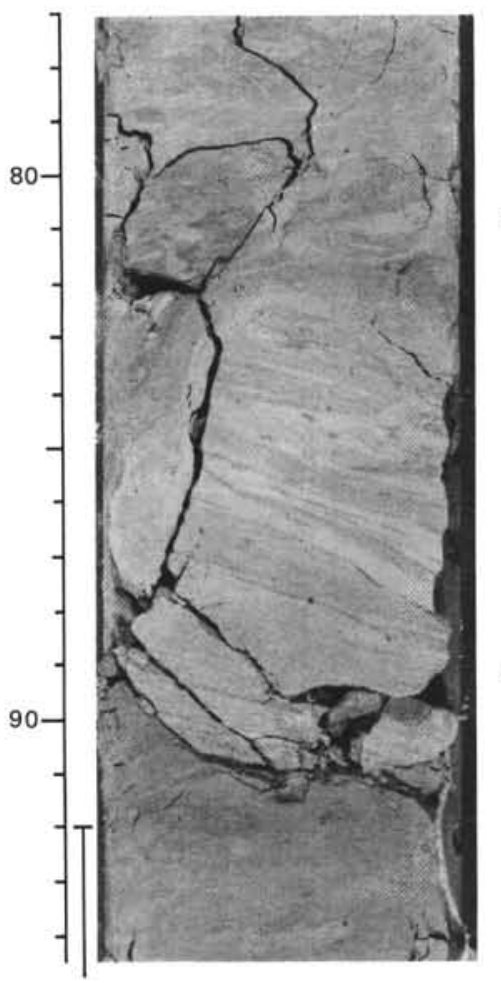

1

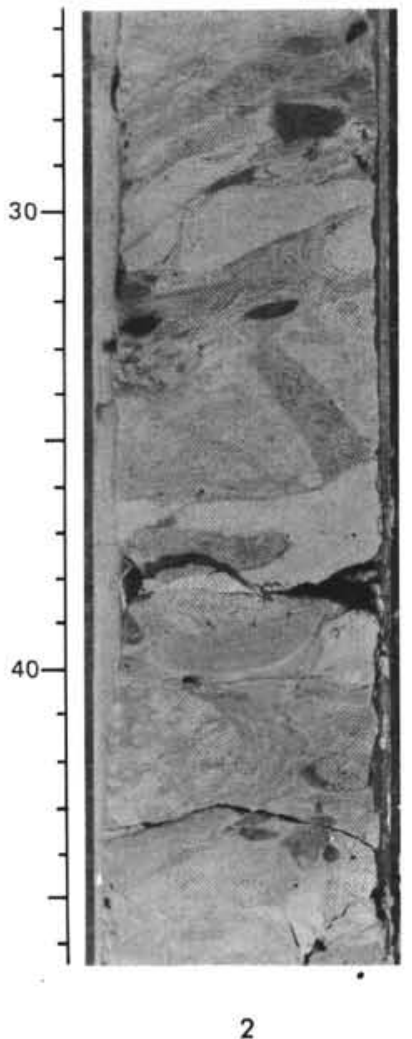

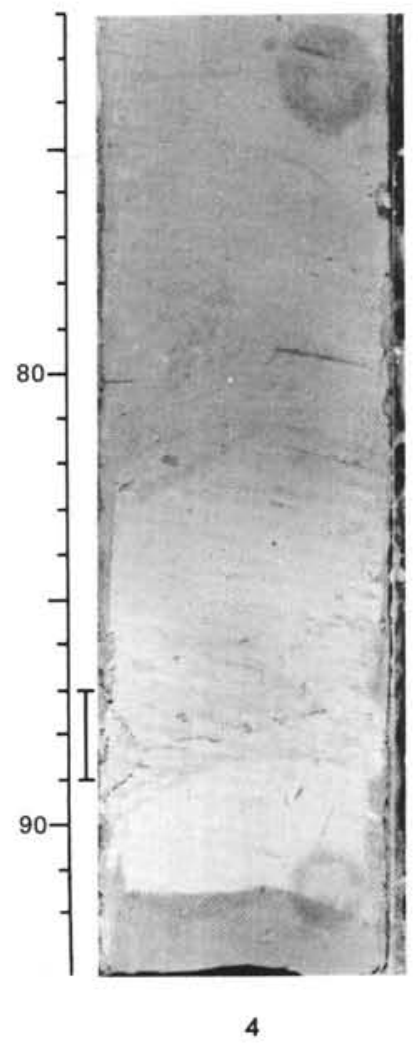

